CERIAS Tech Report 2006-25

\title{
FORMAL FOUNDATIONS FOR HYBRID HIERARCHIES IN GTRBAC
}

by James B. Joshi, E. Bertino. A. Ghafoor

Center for Education and Research in

Information Assurance and Security,

Purdue University, West Lafayette, IN 47907-2086 


\title{
Formal Foundations for Hybrid Hierarchies in GTRBAC
}

\author{
JAMES B D JOSHI \\ School of Information Sciences, University of Pittsburgh, USA \\ ELISA BERTINO \\ CERIAS \& Computer Science, Purdue University, USA \\ ARIF GHAFOOR \\ School of Electrical and Computer Engineering, Purdue University, USA
}

\begin{abstract}
A role hierarchy defines semantics related to permission acquisitions and role activations through role-role relationships. It can be utilized for efficiently and effectively structuring functional roles of an organization having related access control needs. Temporal constraints on role enablings and role activations can have various implications on such a role hierarchy. The focus of this paper is the analysis of hybrid role hierarchies in the context of the Generalized Temporal Role Based Access Control (GTRBAC) model that allows specification of a comprehensive set of temporal constraints on role, user-role assignments and role-permission assignments. We introduce the notion of uniquely activable set (UAS) associated with a role hierarchy that indicates the access capabilities of a user resulting from his membership to a role in the hierarchy. Identifying such a role set is essential while making an authorization decision about whether or not a user should be allowed to activate a particular combination of roles in a single session. Furthermore, when separation-of-duty ( $\mathrm{SoD}$ ) constraints are present in the system, it is also essential to ensure that there are no role combinations that can be allowed to be activated in a single user session. In other words, knowledge about UAS can be used to facilitate enforcement of the principle of least privilege. Because of the separation of permission inheritance and role activation semantics in GTRBAC, a hybrid hierarchy that allows different hierarchy types to coexist, can give rise to a complex semantics and identifying what role combinations can be allowed to be activated in a session for a user may not be straight forward. We formally show how UAS can be determined for a hybrid hierarchy. Furthermore, within a hybrid hierarchy, various hierarchical relations may be derived between an arbitrary pair of roles. We present a set of inference rules that can be used to generate all the possible derived relations that can be inferred from a specified set of hierarchical relations and show that the set of these inference rules is sound and complete. Another key issue we address in this paper is that of the evolution of role hierarchies through hierarchical transformations. We present an analysis of hierarchy transformations with respect to role addition, deletion and partitioning, and show how various cases of these transformations allow the original permission acquisition and role activation semantics to be managed. The formal results presented here provide a basis for developing efficient security administration and management tools.
\end{abstract}

\section{INTRODUCTION}

Role based access control (RBAC) has emerged as a promising alternative to traditional discretionary and mandatory access control (DAC and MAC) models [Giuri 1995], [Giuri 1996], [Joshi et al. 2001], [Nyanchama and Osborn 1999], [Osborn et al. 2000], [Sandhu et al. 1996], [Koch et al. 2002], which have inherent limitations [Joshi et al. 2001]. Several beneficial features such as policy neutrality, support for least privilege and efficient access control management are associated with RBAC models [Ferraiolo et al. 1993], [Joshi et al. 2001], [Sandhu et al. 1996]. Such features make 
RBAC better suited for handling access control requirements of diverse organizations. RBAC models have also been found suitable for addressing security issues in the Internet environment [Barkley et al. 1997], [Joshi et al. 2001], [Park et al. 2001] and show promise for newer heterogeneous multi-domain environments that raise serious concerns related to access control across domain boundaries [Biskup et al. 1998], [Joshi et al. 2001].

An essential part of an RBAC model is the notion of a role hierarchy. Role hierarchies play a crucial role in authorization management and administration [Moffett 1998], [Sandhu 1996], [Sandhu 1998], [Jaeger and Tidswell 2001] and in the succinct RBAC representations of DAC and MAC policies [Osborn et al. 2000]. When two roles are hierarchically related, one is called the senior and the other the junior. In the most commonly accepted RBAC96 family of models [Sandhu et al. 1996], a senior role and its junior roles are related by an inheritance relation that has two semantic parts: permission-inheritance (also called permissionusage [Sandhu 1998]) and role-activation semantics. Permission-inheritance semantics allows a senior role to inherit all the permissions assigned to its junior roles, whereas the role-activation semantics allows all the users assigned to a senior role to activate its junior roles. The RBAC96 models use the combined hierarchy semantics that allows both the permission-inheritance and the role-activation semantics. This significantly reduces assignment overhead, as the permissions need only be assigned to junior roles [Sandhu 1998], [Moffett 1998]. Sandhu showed that, under the combined hierarchy semantics, certain separation of duty (SoD) constraints cannot be defined on hierarchically related roles, thus, restricting its effectiveness in supporting a broader set of fine-grained constraint specification, and, in particular, in representing MAC policies [Sandhu 1998]. To address such shortcomings of RBAC96, Sandhu [Sandhu 1998] has proposed the ER-RBAC96 model that incorporates a distinction between a usage hierarchy that applies only the permission-inheritance semantics and an activation hierarchy that uses the combined hierarchy semantics. Later, Joshi et al. [Joshi et al. 2002] have established a clear distinction between the three role hierarchies: permission-inheritance-only hierarchy ( $I$-hierarchy), activation-only hierarchy ( $A$-hierarchy), and the combined permission-inheritance and activation hierarchy (IA-hierarchy). The need for different semantics for hierarchical relations has also been recognized by Moffet et al. in [Moffett 1998], [Moffett and Lupu 1999]. In particular, they have identified the need for three types of organizational hierarchies - is a hierarchy, activity hierarchy and supervision hierarchy - in order to address the needs of control principles in an organization, such as SoD, decentralization of control and supervision and review [Moffett 1998], [Moffett and Lupu 1999]. Use of a combined hierarchy semantics has been found to limit a hierarchy in achieving these organizational control goals and, hence, to address such control requirements it is desirable to configure a hybrid role hierarchy that allows different hierarchical relations among roles [Joshi et al. 2002]. Such a hybrid hierarchy is provided as part of the recently proposed Generalized Temporal RBAC model [Joshi et al. 2005b] and it is able to support a variety of combinations of inheritance and activation semantics.

Another relevant functionality in access control is that of time-constraining ac- 

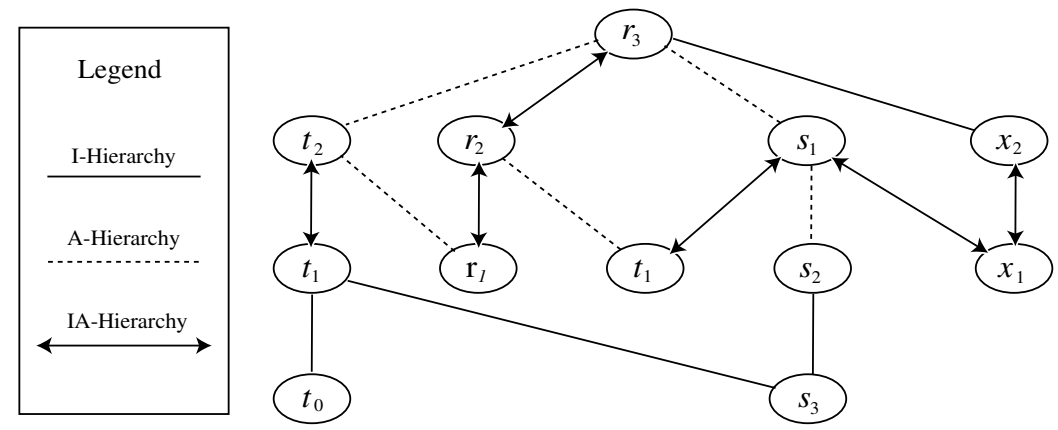

Fig. 1. An example hybrid hierarchy

cesses to resources for controlling time-sensitive activities in an application, for instance, in a workflow management system (WFMS) [Bertino and Ferrari 1999], where various workflow tasks, each having some timing constraints, need to be executed in some order. Bertino et al.'s Temporal RBAC model (TRBAC) provides the first framework for modeling of time-constrained access policies [Bertino et al. 2001]. The GTRBAC model extends the TRBAC model and incorporates a set of language constructs for specifying a large set of periodicity and duration constraints including those on role enabling, user-role and role-permission assignments, and role activations. An important issue in the GTRBAC model is the interplay between the temporal constraints and role hierarchies, which has been first addressed in [Joshi et al. 2002]. Accordingly, Joshi et al. identify various subtypes of the $I, A$ and $I A$-hierarchies that capture temporal semantics of a hierarchy in presence of temporal constraints on roles. In presence of a hybrid hierarchy containing multiple hierarchy types, a user may be able to activate different sets of junior roles in a session. Sets of roles that can be activated or permissions that can be acquired by a user at a particular time indicate the overall access capabilities of the user. From the perspective of the principle of least privilege, it may be necessary to ensure that such activable sets of roles do not result in granting users unnecessary access capabilities. Determining such sets can become very complex in presence of a hybrid hierarchy. Furthermore, it is essential to know what indirect relations may exist between roles that are not directly related so that when modifications are made to the hierarchy, original relations can be maintained if possible. For example, consider the relatively simple hybrid hierarchy of Fig. 1. Here, determining the sets of roles that can be activated in a single session by a user assigned only to the role $r_{3}$ is not straightforward. Similarly, when we delete the role $s_{1}$, we need to make sure that the original relations between $r_{3}$ and $t_{1}, r_{3}$ and $s_{2}$ or $r_{3}$ and $x_{1}$ are retained.

Flexible models, like GTRBAC, need formal tools for an efficient security administration and management. In this paper, we present a formal basis for analyzing hybrid hierarchies in GTRBAC. The contributions of this paper include the following:

-We define the notion of uniquely activable set of a hierarchy that can be used by security administrators for determining access capabilities that a user can 
obtain from a role hierarchy in a single session and show formally how it can be determined in a hybrid temporal role hierarchy.

-We introduce a set of inference rules that allows inferring the hierarchical relationships between an arbitrary pair of roles that are not directly related and show that it is sound and complete.

-We develop a set of hierarchy transformation algorithms to assist in administering role hierarchies when the roles are added, deleted or modified.

The paper is organized as follows. In Section 2, we overview the GTRBAC model. In Section 3, we introduce the three basic hierarchical relations that can exist on a set of roles followed by their subtypes. In Section 4, we introduce the notion of uniquely activable set and a formal technique for determining it. In section 5 , we introduce a set of inference rules for inferring derived relations between an arbitrary pair of roles. In Section 6 , we introduce hierarchy transformation algorithms. Related work is discussed in Section 7. Conclusions and future work are presented in Section 8. Appendix A provides the proofs of formal results in section 4 whereas Appendix B provides the proofs of formal results of section 5.

\section{GENERALIZED TEMPORAL ACCESS CONTROL MODEL (GTRBAC)}

The GTRBAC model introduces the separate notion of role enabling and role activation, and provides constraints and event expressions associated with both. An enabled role indicates that a valid user can activate it, whereas an activated role indicates that at least one user has activated it. The GTRBAC model allows the specification of the following set of constraints:

(1) Temporal constraints on role enabling/disabling: These constraints allow the specification of intervals and durations in which a role is enabled. When a role is enabled, the permissions assigned to it can be acquired by a user by activating it. When a duration constraint is specified, the enabling/disabling of a role is initiated by a constraint enabling event that results from the firing of a trigger or through an administrator initiated run-time event.

(2) Temporal constraints on user-role and role-permission assignments: These constraints allow specifying intervals and durations in which a user or a permission is assigned to a role.

(3) Activation constraints: These constraints allow specification of restrictions on the activation of a role. These include, for example, specifying the total duration for which a user may activate a role, or the number of concurrent activations of a role at a particular time.

(4) Run-time events: A set of run-time events allows an administrator to dynamically initiate GTRBAC events, or enable duration or activation constraints. Another set of run-time events allow users to request activation or deactivation of a role.

(5) Constraint enabling expressions: The GTRBAC model includes events that enable or disable duration and role activation constraints mentioned earlier.

(6) Triggers: The GTRBAC triggers allow expressing dependencies among events. 
Table I. Constraint Expressions

\begin{tabular}{|c|c|c|c|}
\hline $\begin{array}{l}\text { Constraint } \\
\text { categories }\end{array}$ & \multicolumn{2}{|c|}{ Constraints } & Expression \\
\hline \multirow{3}{*}{$\begin{array}{l}\text { Periodicity } \\
\text { Constraint }\end{array}$} & \multicolumn{2}{|c|}{ User-role assignment } & $\left(I, P, p r:\right.$ assignU $/$ deassign $_{U} r$ to $\left.u\right)$ \\
\hline & \multicolumn{2}{|l|}{ Role enabling } & $(I, P, p r:$ enable/disable $r)$ \\
\hline & \multicolumn{2}{|c|}{ Role-permission assignment } & $\left(I, P, p r:{\left.\text { assign } P / \text { deassign }_{P} p \text { to } r\right)}\right.$ \\
\hline \multirow{3}{*}{$\begin{array}{l}\text { Duration } \\
\text { Constraints }\end{array}$} & \multicolumn{2}{|c|}{ User-role assignment } & $\left([(I, P) \mid D], D_{U}, p r:\right.$ assign $_{U} /$ deassign $_{U} r$ to $\left.u\right)$ \\
\hline & \multicolumn{2}{|l|}{ Role enabling } & $\left([(I, P) \mid D], D_{R}, p r:\right.$ enable $/$ disable $\left.r\right)$ \\
\hline & \multicolumn{2}{|c|}{ Role-permission assignment } & $\left([(I, P) \mid D], D_{P}, p r:\right.$ assign $_{P} /$ deassign $_{P} p$ to $\left.r\right)$ \\
\hline \multirow{4}{*}{$\begin{array}{l}\text { Duration } \\
\text { Constraints } \\
\text { on Role } \\
\text { Activation }\end{array}$} & \multirow{2}{*}{$\begin{array}{l}\text { Total active } \\
\text { role duration }\end{array}$} & Per-role & $\left([(I, P) \mid D], D_{\text {active }},\left[D_{\text {default }}\right], p r:\right.$ active $\left._{R_{-t o t a l}} r\right)$ \\
\hline & & Per-user-role & $\left([(I, P) \mid D], D_{\text {uactive }}, u, p r:\right.$ active $\left._{U R_{-} \text {total }} r\right)$ \\
\hline & \multirow{2}{*}{$\begin{array}{l}\text { Max role } \\
\text { duration per } \\
\text { activation }\end{array}$} & Per-role & $\left([(I, P) \mid D], D_{\max }, p r:\right.$ active $\left.R_{-\max } r\right)$ \\
\hline & & Per-user-role & $\left([(I, P) \mid D], D_{u \max }, u, p r:\right.$ active $\left._{U} R_{-} \max r\right)$ \\
\hline \multirow{4}{*}{$\begin{array}{l}\text { Cardinality } \\
\text { Constraint } \\
\text { on Role } \\
\text { Activation }\end{array}$} & \multirow{2}{*}{$\begin{array}{l}\text { Total no. of } \\
\text { activations }\end{array}$} & Per Role & $\left([(I, P) \mid D], N_{\text {active }},\left[N_{\text {default }}\right], p r:\right.$ active $\left._{R_{-} n} r\right)$ \\
\hline & & Per-user-role & $\left([(I, P) \mid D], N_{\text {uactive }}, u, p r:\right.$ active $\left._{U R_{-} n} r\right)$ \\
\hline & \multirow{2}{*}{$\begin{array}{l}\text { Max. no. of } \\
\text { concurrent } \\
\text { activations }\end{array}$} & Per-role & $\left([(I, P) \mid D], N_{\max },\left[N_{\text {default }}\right], p r:\right.$ active $\left._{R_{-} \text {con }} r\right)$ \\
\hline & & Per-user-role & $\left([(I, P) \mid D], N_{u \max }, u, p r:\right.$ active $\left._{U R_{-} \text {con }} r\right)$ \\
\hline Trigger & \multicolumn{3}{|c|}{$E_{1}, \ldots, E_{n}, C_{1}, \ldots, C_{k} \rightarrow p r: E$ after $\Delta t$} \\
\hline $\begin{array}{l}\text { Constraint } \\
\text { Enabling }\end{array}$ & \multicolumn{3}{|c|}{ pr:enable/disable c where $c\left(\left\{\left(D, D_{x}, p r: E\right),(C),(D, C)\right\}\right)$} \\
\hline \multirow{5}{*}{$\begin{array}{l}\text { Run-time } \\
\text { Requests }\end{array}$} & \multicolumn{2}{|c|}{ Users' activation request } & $(s:($ de)activate $r$ for $u$ after $\Delta t))$ \\
\hline & \multirow{4}{*}{\multicolumn{2}{|c|}{$\begin{array}{l}\text { Administrator's run-time } \\
\text { request }\end{array}$}} & $\left(p r:\right.$ assign $_{U} /$ de - assign $_{U} r$ to $u$ after $\left.\Delta t\right)$ \\
\hline & & & $(p r:$ enable/disable $r$ after $\Delta t)$ \\
\hline & & & $\left(p r: \operatorname{assign}_{P} /\right.$ de - assign $_{P} p$ to $r$ after $\left.\Delta t\right)$ \\
\hline & & & $(p r:$ enable/disable $c$ after $\Delta t)$ \\
\hline
\end{tabular}

Table I summarizes the constraint types and expressions of the GTRBAC model. The periodic expression used in the constraint expressions is of the form $(I, P)$, where $P$ is an expression denoting an infinite set of periodic time instants, and $I=[$ begin, end $]$ is a time interval denoting the lower and upper bounds that are imposed on instants in $P$. The function $\operatorname{Sol}(I, P)$ is used to denote all the time instants in $(I, P)$. $D$ expresses the duration specified for a constraint. In the duration and role activation constraint expressions, $D_{x}$ and $N_{x}$ indicate the duration and cardinality values. If the subscript $x$ starts with $u$, then it is a per-user-role constraint otherwise it is a per-role constraint. For instance, $D_{\text {active }}$ indicates the duration for which the specified role can be active, whereas, $D_{\text {uactive }}$ indicates the duration for which the specified user may activate the specified role. The following example illustrates the specification of a GTRBAC policy. For more details on the GTRBAC mode, we refer the readers to [Joshi et al. 2005b].

Example 1: Table II contains the GTRBAC policy for a hospital. The periodicity constraint $1 a$ specifies the enabling times of DayDoctor and NightDoctor roles. For simplicity, we use DayTime and NightTime instead of their $(I, P)$ forms. The periodicity constraint $1 b$ allows the DayDoctor role to be assigned to Adams on Mondays, Wednesdays and Fridays, and to Bill on Tuesdays, Thursdays, Saturdays and Sundays. Similarly, Alice and Ben are assigned to the NightDoctor role on the different days of the week. Furthermore, the assignment in $1 c$ allows Carol 
Table II. An Example GTRBAC access policy for a medical information System

\begin{tabular}{|c|c|c|}
\hline \multirow{6}{*}{1} & a. & (DayTime, enable DayDoctor), (NightTime,enable NightDoctor) \\
\hline & \multirow{4}{*}{ b. } & $\left((\mathrm{M}, \mathrm{W}, \mathrm{F})\right.$, assign $_{U}$ Adams to DayDoctor $)$ \\
\hline & & $\left((\mathrm{T}, \mathrm{Th}, \mathrm{S}, \mathrm{Su})\right.$, assign $_{U}$ Bill to DayDoctor $)$ \\
\hline & & $(\mathrm{M}, \mathrm{W}, \mathrm{F})$, assign $_{U}$ Alice to NightDoctor $)$ \\
\hline & & $\left((\mathrm{T}, \mathrm{Th}, \mathrm{S}, \mathrm{Su})\right.$, assign $_{U}$ Ben to NightDoctor $)$ \\
\hline & c. & $\left([10 \mathrm{am}, 3 \mathrm{pm}]\right.$, assign $_{U}$ Carol to DayDoctor $)$ \\
\hline \multirow{2}{*}{2} & a. & (assign $_{U}$ Ami to NurseInTraining); (assign $n_{U}$ Elizabeth to DayNurse) \\
\hline & b. & (6 hours, 2 hours, enable NurseInTraining) \\
\hline \multirow{6}{*}{3} & a. & (enable DayNurse $\rightarrow$ enable $c 1$ ) \\
\hline & b. & (activate DayNurse for Elizabeth $\rightarrow$ enable NurseInTraining after $10 \mathrm{~min}$ ) \\
\hline & \multirow{2}{*}{ c. } & (enable NightDoctor $\rightarrow$ enable NightNurse after $10 \mathrm{~min}$ ); \\
\hline & & $($ disable NightDoctor $\rightarrow$ disable NightNurse after $10 \mathrm{~min})$ \\
\hline & \multirow{2}{*}{ d. } & (enable DayDoctor $\rightarrow$ enable DayNurse after $10 \mathrm{~min}$ ); \\
\hline & & (disable DayDoctor $\rightarrow$ disable DayNurse after $10 \mathrm{~min})$ \\
\hline
\end{tabular}

to assume the DayDoctor role everyday between 10am and 3pm. In $2 a, A m i$ and Elizabeth are assigned to roles NurseInTraining and DayNurse respectively with no temporal restriction, i.e., the assignment is valid at all times. $2 b$ specifies a duration constraint of 2 hours on the enabling time of the NurseInTraining role, but this constraint is valid for only 6 hours after the constraint $c 1$ has been enabled. Because of this, $A m i$ will be able to activate the NurseInTraining role at the most for two hours whenever the role is enabled. In row 3, we have a set of triggers. Trigger $3 a$ indicates that constraint $c 1$ is enabled when the DayNurse is enabled, which means, now, the NurseInTraining role can be enabled within the next 6 hours. Trigger $3 b$ indicates that $10 \mathrm{~min}$ after Elizabeth activates the DayNurse role, the NurseInTraining role is enabled for a period of 2 hours. This shows that a nurse in training will have access to the system only if Elizabeth is present in the system, that is, she may be acting as a training supervisor. It is possible that Elizabeth activates the DayNurse role a number of times in 6 hours after the DayNurse role has been enabled, and each time the NurseInTraining role will also be enabled if these activations (by Elizabeth) are more than 2 hours apart. This will allow Ami to activate the NurseInTraining role a number of times. The remaining triggers in row 3 show that the DayNurse and NightNurse roles are enabled (disabled) 10 min after the DayDoctor and NightDoctor roles are enabled (disabled).

\section{TEMPORAL ROLE HIERARCHIES}

In earlier work, we introduced the following three hierarchy types: permissioninheritance-only hierarchy ( $I$-hierarchy), role-activation-only hierarchy ( $A$-hierarch y) and the combined permission-inheritance-activation hierarchy (IA-hierarchy) [Joshi et al. 2002]. Table III shows the notation for various predicates used in the definitions of these hierarchies. Predicates $\operatorname{enabled}(r, t)$, assigned $(u, r, t)$ and assigned $(p, r, t)$ refer to the status of roles, user-role and role-permission assignments at time $t$. Predicate can_activate $(u, r, t)$ indicates that user $u$ can activate role $r$ at time $t$. This implies that user $u$ is implicitly or explicitly assigned to 
role $r$. active $(u, r, s, t)$ indicates that role $r$ is active in user $u$ 's session $s$ at time $t$ whereas, acquires $(u, p, s, t)$ implies that $u$ acquires permission $p$ at time $t$ in session $s$. The axioms below capture the key relationships among these predicates and identify precisely the permission-acquisitions and role-activation semantics allowed in GTRBAC [Joshi et al. 2002].

Table III. Various status predicates

\begin{tabular}{|l|l|}
\hline Predicate & Meaning \\
\hline enabled $(r, t)$ & Role $r$ is enabled at time $t$ \\
\hline$u \_a s s i g n e d(u, r, t)$ & User $u$ is assigned to role $r$ at time $t$ \\
\hline$p \_a s s i g n e d(p, r, t)$ & Permission $p$ is assigned to role $r$ at time $t$ \\
\hline can_activate $(u, r, t)$ & User $u$ can activate role $r$ at time $t$ \\
\hline can_acquire $(u, p, t)$ & User $u$ can acquire permission $p$ at time $t$ \\
\hline can_be_acquired $(p, r, t)$ & Permission $p$ can be acquired through role $r$ at time $t$ \\
\hline active $(u, r, s, t)$ & Role $r$ is active in user $u$ 's session $s$ at time $t$ \\
\hline acquires $(u, p, s, t)$ & User $u$ acquires permission $p$ in session $s$ at time $t$ \\
\hline
\end{tabular}

Axioms: If $r \in$ Roles, $u \in$ Users, $p \in$ Permissions, $s \in$ Sessions, and time instant $t \geqslant 0$, the following implications hold:

(1) p_assigned $(p, r, t) \rightarrow$ can_be_acquired $(p, r, t)$

(2) u_assigned $(u, r, t) \rightarrow$ can_activate $(u, r, t)$

(3) can_activate $(u, r, t) \wedge$ can_be_acquired $(p, r, t) \rightarrow$ can_acquire $(u, p, t)$

(4) $\operatorname{active}(u, r, s, t) \wedge$ can_be_acquired $(p, r, t) \rightarrow \operatorname{acquires}(u, p, s, t)$

Axiom (1) states that if a permission is assigned to a role, then it can be acquired through that role. Axiom (2) states that all users assigned to a role can activate that role. Axiom (3) states that if a user $u$ can activate a role $r$, then all the permissions that can be acquired through $r$ can be acquired by $u$. Similarly, axiom (4) states that if there is a user session in which a user $u$ has activated a role $r$ then $u$ acquires all the permissions that can be acquired through role $r$. We note that axioms (1) and (2) indicate that permission-acquisition and role-activation semantics is governed by explicit user-role and role-permission assignments.

\subsection{Formal Definitions of Temporal Role Hierarchies}

Semantically, the use of a role hierarchy is to extend the possibility of permissionacquisition and role-activation semantics beyond the explicit assignments as indicated by the definitions below [Joshi et al. 2002]. The GTRBAC model's constraint enabling/disabling expressions can be used to specify when a hierarchical relation can be enabled/disabled. Hence, if $h$ is a hierarchical relation, we write "enable/disable h" to enable/disable the relation. This allows administrators to dynamically change, if needed, the hierarchical relationships on a set of roles through periodicity or duration constraints, run-time requests and triggers. The following definitions do not consider the enabling times of the hierarchically related roles, and hence the hierarchies are termed unrestricted. 


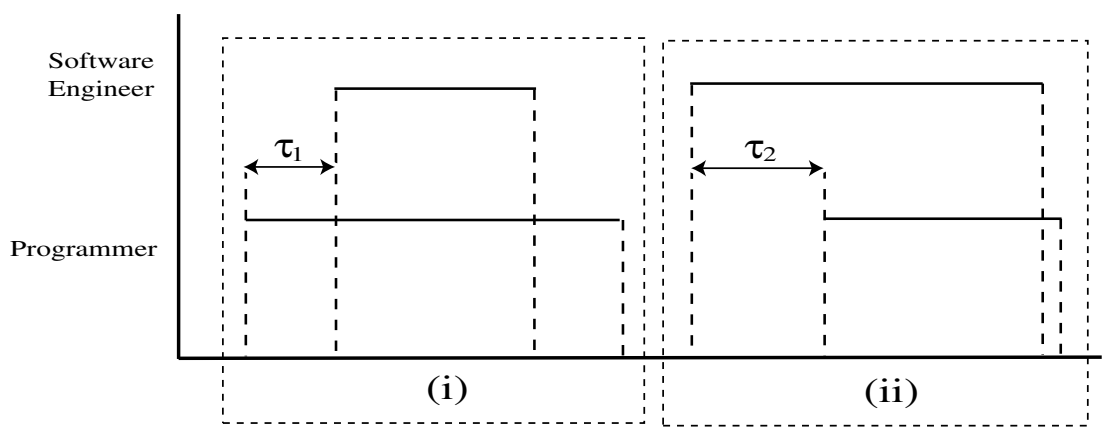

Fig. 2. Enabling intervals of Software Engineer and Programmer Roles

Definition 3.1. (Unrestricted I-hierarchy)[Joshi et al. 2002]: Let $x$ and $y$ be roles such that $\left(x \geqslant_{i} y\right)$, that is, $x$ has an inheritance-only relation over $y$ at time t. Then the following holds:

$$
\forall p,(x \geqslant i y) \wedge \text { can_be_acquired }(p, y, t) \rightarrow \operatorname{can\_ be\_ acquired}(p, x, t)
$$

Definition 3.2. (Unrestricted A-hierarchy) [Joshi et al. 2002]: Let $x$ and $y$ be roles such that $\left(x \geqslant_{a} y\right)$, that is, $x$ has an activation-only relation over $y$ at time t. Then the following holds:

$$
\forall u,\left(x \geqslant{ }_{a} y\right) \wedge \text { can_activate }(u, x, t) \rightarrow \text { can_activate }(u, y, t)
$$

Definition 3.3. (Unrestricted IA-hierarchy) [Joshi et al. 2002]: Let $x$ and $y$ be roles such that $(x \geqslant y)$, that is, $x$ has a general inheritance relation over $y$ at time $t$. Then the following holds: $(x \geqslant y) \leftrightarrow(x \geqslant a y) \wedge(x \geqslant i y)$.

In the definitions above, $x$ is said to be a senior of $y$, and conversely $y$ is said to be a junior of $x$. Thus, if $\left(x \geqslant_{i} y\right)$, the permissions that can be acquired through $x$ include all the permissions assigned to $x$ (by axiom (1)) and all the permissions that can be acquired through role $y$ (by $c_{1}$ ). Note that the axioms and condition $c_{1}$ do not allow $u$, a user assigned to $x$ only, to activate $y$. Condition $c_{2}$ states that if user $u$ can activate role $x$, and $x$ has $A$-relation over $y$, then he can activate role $y$ also, even if $u$ is not explicitly assigned to $y$. As condition $c_{1}$ does not apply to an $A$-hierarchy, $u$ cannot acquire $y$ 's permissions by just activating $x$. The $I A$-hierarchy is the most common form of hierarchy. On a given set of roles, various inheritance relations may be defined. Therefore, we require that the following consistency property be satisfied in a role hierarchy in order to ensure that senior-junior relationship between two roles in one type of hierarchy is not reversed in another. Note that all three hierarchies are transitive.

Property 1 (Consistency of hierarchies) [Joshi et al. 2005a]: Let $\left\langle f_{1}\right\rangle,\left\langle f_{2}\right\rangle \in$ $\left\{\geqslant_{i}, \geqslant_{a}, \geqslant\right\}$. Let $x$ and $y$ be two distinct roles such that $\left(x\left\langle f_{1}\right\rangle y\right) ;$ then the condition $\neg\left(y\left\langle f_{2}\right\rangle x\right)$ must hold.

In what follows, we will always assume that hierarchies are consistent. When we consider the enabling times of hierarchically related roles, we obtain weakly 
restricted and strongly restricted forms of the hierarchies. Their meaning is exemplified by the diagrams in Fig. 2, dealing with two hierarchically related roles Software Engineer and Programmer - hierarchically related. Of those two roles, only one is enabled in intervals $\tau_{1}$ and $\tau_{2}$. In a strongly-restricted hierarchy, inheritance is not allowed in these intervals. This is because in this type of hierarchy both roles must be enabled for inheritance to take place. By contrast, in a weaklyrestricted hierarchy, inheritance may be allowed in these intervals. Table IV shows the inheritance properties of restricted and unrestricted hierarchies in $\tau_{1}$ and $\tau_{2}$.

Table IV. Inheritance semantics for the restricted and unrestricted hierarchies

\begin{tabular}{|c|c|c|c|}
\hline \multicolumn{2}{|c|}{$\begin{array}{c}\text { Interval } \tau \rightarrow \\
\downarrow \text { Hierarchy Type }\end{array}$} & $\begin{array}{c}\tau_{1} \\
r_{1} \text { disabled, } r_{2} \text { enabled }\end{array}$ & $\begin{array}{c}\tau_{2} \\
r_{1} \text { enabled, } r_{2} \text { disabled }\end{array}$ \\
\hline \multirow[t]{2}{*}{$I$-hierarchy } & $I_{w}$ & No inheritance in $\tau$ & $\begin{array}{c}\text { Permission-inheritance in } \tau \\
\text { (by activating } r_{2} \text { ) }\end{array}$ \\
\hline & $I_{s}$ & No inheritance in $\tau$ & No inheritance in $\tau$ \\
\hline \multirow[t]{2}{*}{$A$-hierarchy } & $A_{w}$ & $\begin{array}{c}\text { Activation-inheritance in } \tau \\
\text { (by activating } r_{2} \text { ) }\end{array}$ & No inheritance in $\tau$ \\
\hline & $A_{s}$ & No inheritance in $\tau$ & No inheritance in $\tau$ \\
\hline \multirow[t]{2}{*}{$I A$-hierarchy } & $I A_{w}$ & $\begin{array}{c}\text { Activation-inheritance in } \tau \\
\text { (by activating } r_{2} \text { ) }\end{array}$ & $\begin{array}{c}\text { Activation-inheritance in } \tau \\
\text { (by activating } r_{2} \text { ) }\end{array}$ \\
\hline & $I A_{s}$ & No inheritance in $\tau$ & No inheritance in $\tau$ \\
\hline
\end{tabular}

Note: Subscript $w$ stands for weakly-restricted hierarchy and $s$ stands for strongly-restricted hierarchy.

When activation-time restrictions are to be enforced in GTRBAC, different hierarchy types may need to be considered depending upon whether the constraint is user-centric or permission-centric [Joshi et al. 2002]. An activation constraint is user-centric if it is designed to control different aspects of users in the system through role activations; for example, to control the number of users activating a role. An activation constraint is permission-centric if it is aimed at controlling distribution of the permissions through role activations. Joshi et. al. [Joshi et al. 2002 ] show that an $I$ or $I A$-hierarchy is appropriate when an activation constraint is user-centric, whereas an $A$-hierarchy is appropriate when the activation constraint is permission-centric.

\subsection{Examples of Temporal Role Hierarchies}

We illustrate with the examples reported in Fig. 2(i) and Fig. 2(ii) the practical uses of the various kinds of hierarchies. A practical use of such dynamically changing hierarchical relation is in a case where a senior (acting as a supervisor) is allowed to inherit read-only permissions of its juniors as described in Example 3.1. Moffet et. al. [Moffett 1998] has identified such a supervision-review capability as an important organizational control principle.

Example 3.1: Consider the hierarchy in Fig. 3. Here, we see that the SeniorSec urityAdmin role is enabled only in interval $(8 \mathrm{pm}, 11 \mathrm{pm})$. Neither of its junior roles 


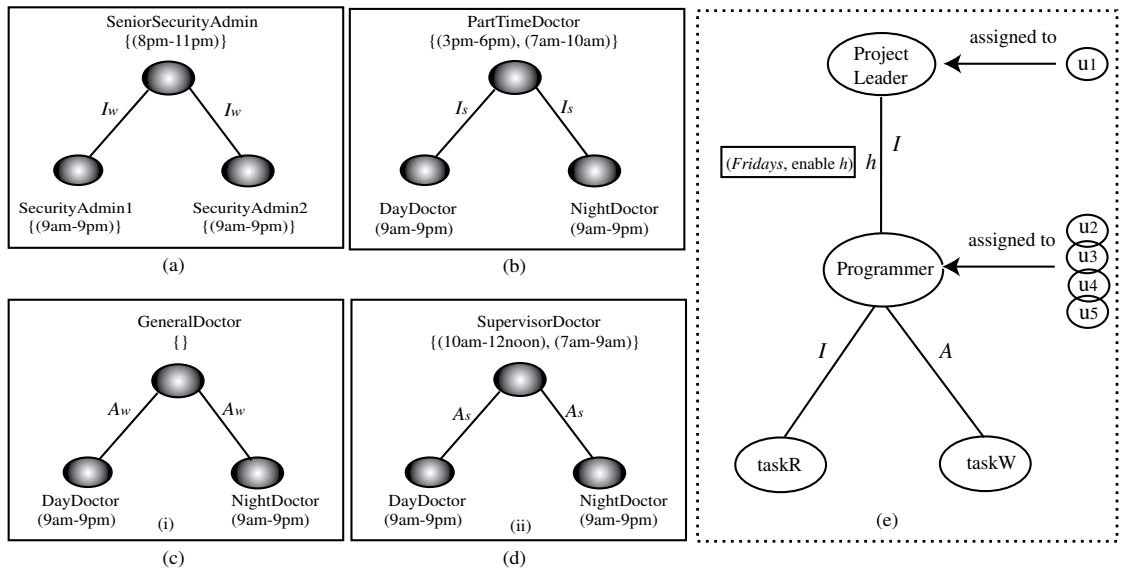

Fig. 3. Hierarchy Examples

is enabled in the entire interval $(8 \mathrm{pm}, 11 \mathrm{pm})$. The $I_{w}$ relation allows a user who activates the SeniorSecurityAdmin role to acquire all the permissions of its junior roles. This may be desirable if SeniorSecurityAdmin role is designed to perform special security operations for checking and maintenance. In such a case, it is reasonable to think that the user assigned to the SeniorSecurityAdmin role will need all the administrative privileges of the junior roles. The temporal restrictions on SecurityAdmin1 and SecurityAdmin2 restrict the users assigned to them to carry out corresponding system administration activities only in the specified intervals. However, here, the user assigned to SeniorSecurityAdmin cannot assume the role of the junior roles SecurityAdmin1 and SecurityAdmin2. To remove this limitation, we can use the $I A_{w}$-hierarchy instead. The hierarchy in Fig. $3(b)$, on the other hand, is of type $I$. The senior role is the PartTimeDoctor role, which has two intervals in which it can be enabled, $(3 \mathrm{pm}, 6 \mathrm{pm})$ and $(7 \mathrm{am}, 10 \mathrm{am})$. If a user activates the PartTimeDoctor role in the first interval, according to the $I_{s}$ relation, he essentially gets all the privileges of the DayDoctor role, as the NightDoctor role is disabled at that time. Now, consider the second interval. We see that it overlaps with the enabling times of the two junior roles. Hence, if the user activates the PartTimeDoctor role in the second interval, he acquires the privileges of only the NightDoctor role in the sub-interval $(7 \mathrm{am}, 9 \mathrm{am})$ and that of only the DayDoctor role in the sub-interval $(9 \mathrm{am}, 10 \mathrm{am})$. Thus, we see that the two different semantics of an inheritance hierarchy can be used to achieve different needs. Again, a part time doctor cannot work as a DayDoctor or a NightDoctor, although, he can acquire the permissions assigned to them. If a user is also to be allowed to use the junior roles, we can use $I A_{s}$-hierarchy instead.

Now, consider Fig. 3(c). Here, we see that there is no interval in which the GeneralDoctor role can be enabled. However, since the activation hierarchy is of type $A_{w}$, any user assigned to the GeneralDoctor role can activate either of the junior roles when they are enabled. In effect, any user assigned to the GeneralDoctor 
role can activate both the DayDoctor and the NightDoctor roles whenever they are enabled. Fig. $3(d)$ illustrates the use of an activation hierarchy of type A. Here, a supervising doctor can assume the SupervisorDoctor role in intervals $(10 \mathrm{am}, 12$ noon $)$ and $(7 \mathrm{am}, 9 \mathrm{am})$. In the first interval, the supervisor will be able to acquire all the privileges of the DayDoctor role by activating it and in the second interval, he will be able to acquire all the privileges of the NightDoctor role by activating it along with the SupervisorDoctor role. The SupervisorDoctor role may simply contain some extra privileges that are required for the supervision task during daytime or nighttime.

Example 3.2: Consider the following requirements for a programming project. A software tool is used for the programming task. The project leader mainly supervises the programming tasks. Only the programmers do the coding. The project leader can only look at the tasks the programmers have carried out on a weekly basis, say on Fridays. Fig. 3(e) depicts the hierarchy that can be generated for achieving the goal. Role TaskR contains the read-only permissions whereas role TaskW contains all the write/modify permissions related to the programming task. The Project Leader role becomes the senior of Programmer role only on Fridays. Note that the users assigned to the Project Leader only inherit TaskR's permissions and cannot acquire any permissions of TaskW.

\section{UNIQUELY ACTIVABLE SET OF A TEMPORAL HIERARCHY}

In this section, we introduce the notion of uniquely activable set(UAS) and present formal results for characterizing it for a hierarchy. The UAS associated with a hierarchy is essentially the set of role sets that can be activated by a user assigned to a role of the hierarchy. In a hierarchy that allows co-existence of the multiple hierarchy types, the permission-inheritance and role-activation semantics can be complex, thus making administration and management of large hierarchies difficult. The UAS gives the role combinations that can be activated by a user in a single session, and thus helps in determining the granularity of permission sets that can be acquired by users through a role in the hierarchy. Thus, UAS is mainly relevant from the perspective of the principle of least privilege. Here, we first determine the UAS characteristics of a monotype hierarchy with only one type of hierarchical relation over the roles, followed by that of a hybrid linear path and then formalize results for the more general role hierarchy. The approach to determining the UAS presented in this section is algorithmic in nature. A mathematical (declarative) way of establishing the UAS is presented in Appendix C. We then introduce the notion of acquisition equivalence to characterize equivalent hierarchies in order to address the usefulness of a hybrid hierarchy. Here onwards we will only use the unrestricted forms of hierarchies. Furthermore, although we consider unrestricted forms of temporal hierarchies, the results directly apply to the non-temporal case with the same three different hierarchy types.

\subsection{Computing Uniquely Activable Set Of A Hierarchy}

We represent by $\sqcup(H)$ the uniquely activable set (UAS) of role sets associated with a user assigned to the senior-most role of a hierarchy $H$ at time instant $t$. For a given role set $X=\left\{x_{1}, x_{2}, \ldots, x_{n}\right\}$ and a set of hierarchy relations $[f] \subseteq\left\{\geqslant_{i}, \geqslant_{a}, \geqslant\right\}$, we 
represent a general hierarchy $H$ over $X$ as $(X,[f])$. If $[f]=\{\langle f\rangle\}$ is a singleton set with hierarchy relation $\langle f\rangle$, then we call $H$ a monotype hierarchy and write $(X$, $\langle f\rangle)$, else we call $H$ a hybrid hierarchy. Furthermore, $H$ is a linear path over $X$ if $(X,[f])$ is an ordered sequence of relation $x_{1}\left\langle f_{12}\right\rangle x_{2}\left\langle f_{23}\right\rangle x_{3} \ldots x_{n-1}\left\langle f_{(n-1) n}\right\rangle x_{n}$ where $\left\langle f_{i j}\right\rangle \in[f]$. We represent a monotype linear path as $L=(X,\langle f\rangle)$ and a hybrid linear path as $L h=(X,[f])$. We use $L H$ to represent either $L h$ or $L H$. In this paper, we assume that

(a) the set of permissions assigned to each role in $\operatorname{Roles}(H)$ is distinct, and

(b) for each hierarchy $H$, there is only one senior-most role, indicated by $S_{H}$.

The results can be easily extended to deal with a general hierarchy. We use $J_{H}$ to denote the set of junior-most roles of $H$.

We use notation $P(r)$ to refer to the set of permissions assigned to role $r$. Similarly, given a set $X$ of roles, we use $P(X)$ to denote $\bigcup_{r \in X} P(r)$. Now, we formally define the UAS of a hierarchy as follows.

Definition 4.1. (Uniquely Activable Set of a Hierarchy $\boldsymbol{H})$ : Let $H=(X$, $[f])$ be a hierarchy. Then, the uniquely activable set for a user $u$ assigned only to role $S_{H}, \sqcup(H)$, is the maximal set of role sets $Y_{1}, Y_{2}, \ldots, Y_{m}$, such that

(1) for each $i \in\{1,2, \ldots, m\}, \varnothing \subset Y_{i} \subseteq X$, and all roles in each $Y_{i}$ can be activated in a single session of $u$,

(2) for all pairs $i, j \in\{1,2, \ldots, m\}$ and $i \neq j, P\left(Y_{i}\right) \neq P\left(Y_{j}\right)$, and,

(3) for each $Z \subseteq X$ such that $Z \notin \sqcup(H)$, if $P\left(Y_{i}\right)=P(Z)$ for some $i$, then $\left(\left|Y_{i}\right|<|Z|\right)$; where $|A|$ denotes the cardinality of set $A$.

Note that each element $Y_{i}$ is a subset of $X$. Condition (2) indicates that each role set of $\bigsqcup(H)$ is unique in terms of the permissions that can be acquired through its roles. Condition (3) considers the possibility of different role sets associated with the same set of permissions. In such a case $\bigsqcup(H)$ contains the role set that has the least number of roles. Conditions (2) and (3) prevent a pair of senior and junior roles, e.g. of an $I A$-hierarchy, to be in a role set of $\bigsqcup(H)$. For instance, if relation $(x \geqslant y)$ is in $H$, then the set $\{x\}$ and not $\{x, y\}$ will be in $\bigsqcup(H)$, as $P(x)=P(\{x, y\})$. The $\bigsqcup(H)$ values for $I, A$ and $I A$-hierarchy can differ significantly because of the difference in permission-inheritance and role-activation semantics associated with them.

As a hybrid linear path may have different types of hierarchical relations it can be decomposed into a set of monotype linear paths. The following definition formalizes the notion of monotype decomposition of a hybrid linear path (MDHP).

We denote the senior-most and the junior-most roles of a hybrid hierarchy $L h$ as $S_{L h}$ and $J_{L h}$.

Definition 4.2. (Monotype Decomposition of Hybrid Path - MDHP): Let $L h=(X,[f])$ be a hybrid linear path over role set $X$. Then Lh can be decomposed into an ordered set $L h=\left(L_{1}, L_{2}, \ldots, L_{n}\right)$ with $X=X_{1} \bigcup X_{2} \bigcup . . \bigcup X_{n}$, such that $L_{i}=\left(X_{i},\left\langle f_{i}\right\rangle\right)$ is a monotype linear path, and the following conditions hold:

(1) for all $i \in\{1, \ldots, n-1\}$, (i) $\left\langle f_{i}\right\rangle \neq\left\langle f_{i+1}\right\rangle$, and (ii) $X_{i} \cap X_{i+1}=\left\{J_{L_{i}}\right\}=$ $\left\{S_{L_{(i+1)}}\right\}$, and

(2) for all $i \in\{1, \ldots, n\}$ and $(i+1<j \leqslant n)$ or $(1 \leqslant j<i-1), X_{i} \cap X_{j}=\phi$, 


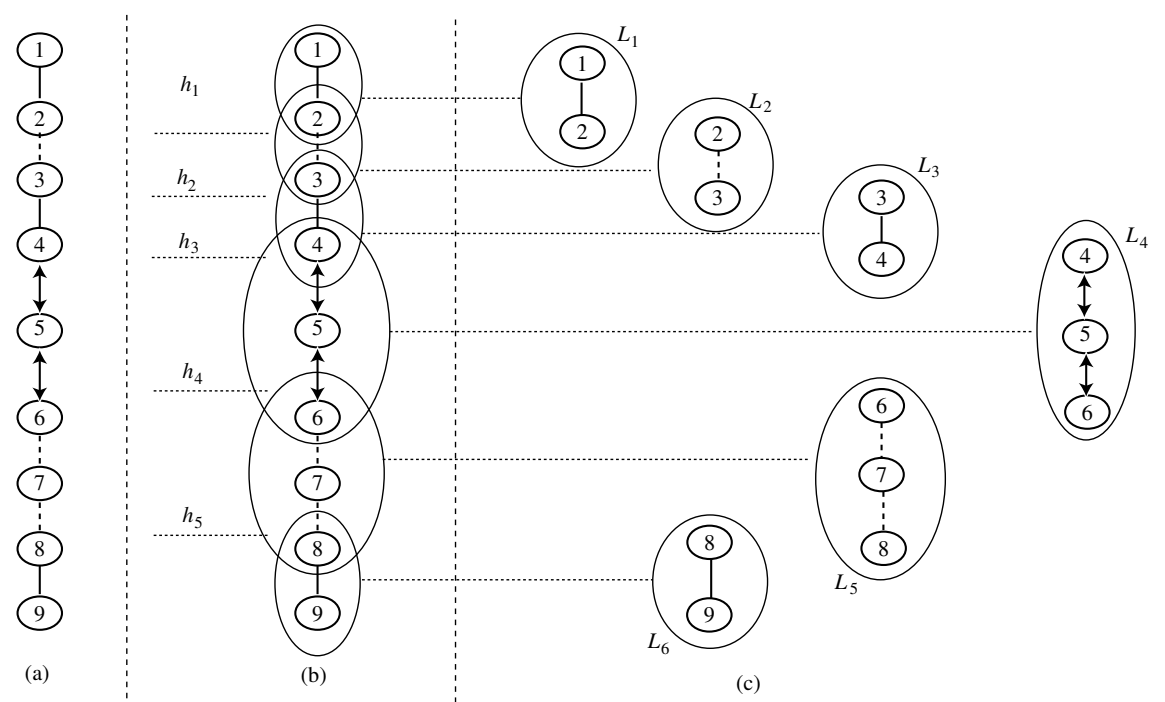

Fig. 4. Complete horizontal partition of a hybrid linear path

Here, $\left(L_{1}, L_{2}, \ldots, L_{n}\right)$ is minimal. Lh can also be written as $\left(L_{1}, L h^{\prime}\right),\left(L h^{\prime}, L_{n}\right)$, $\left(L h_{x}, L h_{y}\right)$, etc., each of which is an MDHP of Lh, not necessarily minimal.

It is easy to see that $S_{L h}=S_{L_{1}}$, and $J_{L h}=J_{L_{n}}$. As indicated, we will use $L$ to represent a monotype linear path, $L h$ to represent a hybrid linear path, and $L H$ to mean either of them. $H$ represents any hierarchy. As indicated by definition 4.2 , we can break a hybrid linear path into an ordered set of monotype linear paths. Such an MDHP of a hybrid path allows us to use the $\bigsqcup(H)$ of the monotype linear paths to determine the $\sqcup(H)$ of a hybrid linear path. Note that the minimal MDHP consists of monotype linear paths that are maximal in the sense that combining any consecutive pair of component linear paths will give a component hybrid linear path, as indicated by part (1) of the definition. The use of MDHPs of $L h$ that are not minimal allows expressing a hybrid linear path as a combination of smaller linear paths that may be of hybrid type. Example 4.1 illustrates the decomposition of a hybrid linear path into its monotype components.

Consider the role hierarchy of Fig. 4(a). The complete MDHP of the hybrid linear path is $\left(L_{1}, L_{2}, L_{3}, L_{4}, L_{5}, L_{6}\right)$ as shown in Fig. $4(\mathrm{c})$. We note that if $L_{4}$ is split into $L_{4,1}=(\{4,5\}, I A$-type $)$ and $L_{4,2}=(\{5,6\}, I A$-type $)$, then $L_{1}, L_{2}, L_{3}, L_{4,1}, L_{4,2}, L_{5}, L_{6}$ is not a complete MDHP, as $L_{4,1}$ and $L_{4,2}$ do not satisfy part (1) of definition 4.2 .

In this paper, we also use functions $\operatorname{sub}_{L}(L H)$ and $s u b_{U}(L H)$ that return the lower and upper parts of a linear path $L H$. That is, if

$L H=x_{1}\left\langle f_{12}\right\rangle x_{2}\left\langle f_{23}\right\rangle x_{3} \ldots x_{n-1}\left\langle f_{(n-1) n}\right\rangle x_{n}$, where $\left\langle f_{i(i+1)}\right\rangle \in\left\{\geqslant_{i}, \geqslant_{a}, \geqslant\right\}$. Then, $-\operatorname{sub}_{L}(L H)=x_{2}\left\langle f_{23}\right\rangle x_{3} \ldots x_{n-1}\left\langle f_{(n-1) n}\right\rangle x_{n} ;$ 
$-\operatorname{sub}_{U}(L H)=x_{1}\left\langle f_{12}\right\rangle x_{2}\left\langle f_{23}\right\rangle x_{3} \ldots\left\langle f_{n-2}\right\rangle x_{n-1} ;$ For $L=(x\langle f\rangle y), \operatorname{sub}_{L}(L)=$ $\operatorname{sub}_{U}(L)=\varnothing ;$

$-\operatorname{sub}_{L}(L H)=\operatorname{sub}_{L}\left(L_{1}\right), L_{2}, \ldots, L_{n}$, and,

$-\operatorname{sub}_{U}(L H)=\left\{L_{1}, L_{2}, \ldots, s u b_{U}\left(L_{n}\right)\right\}$, where $L H=\left(L_{1}, L_{2}, \ldots, L_{n}\right)$ is the complete $M D H P$ of $L H$.

Here, $\operatorname{sub}_{L}(L H)$ and $s u b_{U}(L H)$ return the lower and the upper sub-paths of $L H$. $\operatorname{sub}_{L}(x\langle f\rangle y)=\operatorname{sub}_{U}(x\langle f\rangle y)=\varnothing$ indicates that path $(x\langle f\rangle y)$ has no sub-paths.

In other words, if $L H=\left(L_{1}, L_{2}, \ldots L_{n}\right)$ is the minimal MDHP then

$\operatorname{sub}_{L}(L H)=\left(\operatorname{sub}_{L}\left(L_{1}\right), L_{2}, \ldots, L_{n}\right)$, and

$\operatorname{sub}_{U}(L H)=\left(L_{1}, L_{2}, \ldots, \operatorname{sub}_{U}\left(L_{n}\right)\right)$

Because of the different activation semantics associated with each hierarchy type, $\bigsqcup(H)$ associated with each type is also different. The following theorem formally characterizes the $\bigsqcup(H)$ of a monotype hierarchy:

THEOREM 4.1. Let $H=(X,\langle f\rangle)$ be a monotype linear hierarchy defined over role set $X=\left\{x_{1}, x_{2}, \ldots, x_{n}\right\}$ with $\langle f\rangle \in\left\{\geqslant_{i}, \geqslant_{a}, \geqslant\right\}$

Then,

$$
\sqcup(H)= \begin{cases}\left\{S_{H}\right\} & \text { if }\left(\langle f\rangle=\geqslant_{i}\right) \\ \left\{2^{X} \backslash \varnothing\right\} & \text { if }\left(\langle f\rangle=\geqslant_{a}\right) \\ \left\{x_{1}, x_{2}, \ldots, x_{n}\right\} & \text { if }(\langle f\rangle=\geqslant)\end{cases}
$$

The theorem states that $\bigsqcup(H)$ of a linear $I$-hierarchy contains the senior-most role only. $\bigsqcup(H)$ of a linear $A$-hierarchy contains the power set of the role set $X$ without the empty element, i.e., a user assigned to the senior-most role can activate every combination of the roles in the hierarchy. Similarly, $\bigsqcup(H)$ of a linear $I A$-hierarchy contains set elements containing individual roles of the hierarchy. The proof for the theorem follows directly from the transitive properties of the hierarchical relations and the permission inheritance-only and/or role activation-only semantics of the three hierarchies. Example 4.2 illustrates the use of the results of Theorem 4.1

Consider the monotype hierarchies of Fig. 3. For each of the monotype hierarchies in figures $3(a)$ and $3(b)$, the corresponding $\bigsqcup(H)$ s only contain the set with the senior-most role of the hierarchy, as each of them has the senior-most role related to its junior(s) by $I$-relation(s). For hierarchies in figures $3(c)$ and $3(d)$, assuming unrestricted forms in both the cases, instead of the restricted forms indicated in the figures, the UASs are as follows:

Hierarchy of Fig. 3(c): Here,

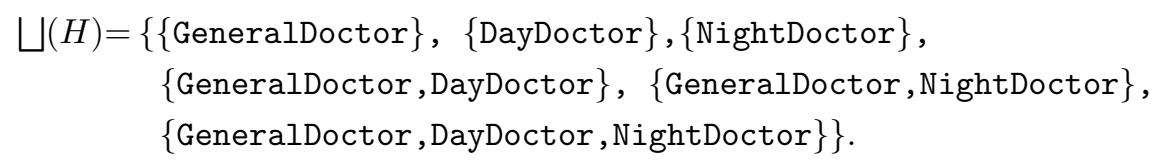


However, GeneralDoctor is never enabled. Furthermore, if we take the periodicity constraints on the roles, we have,

in interval $(9 \mathrm{am}, 9 \mathrm{pm}), \bigsqcup(H)=\{\{$ DayDoctor $\}$,

and in interval $(9 \mathrm{pm}, 9 \mathrm{am}), \bigsqcup(H)=\{\{$ NightDoctor $\}$.

Hierarchy of Fig. 3(d): Here,

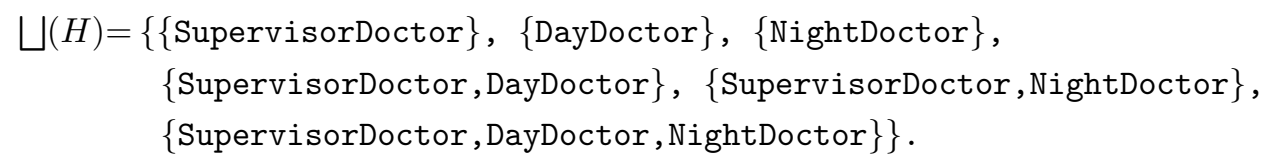

However, the effective $\bigsqcup(H)$ differ the temporal constraints. Hence,

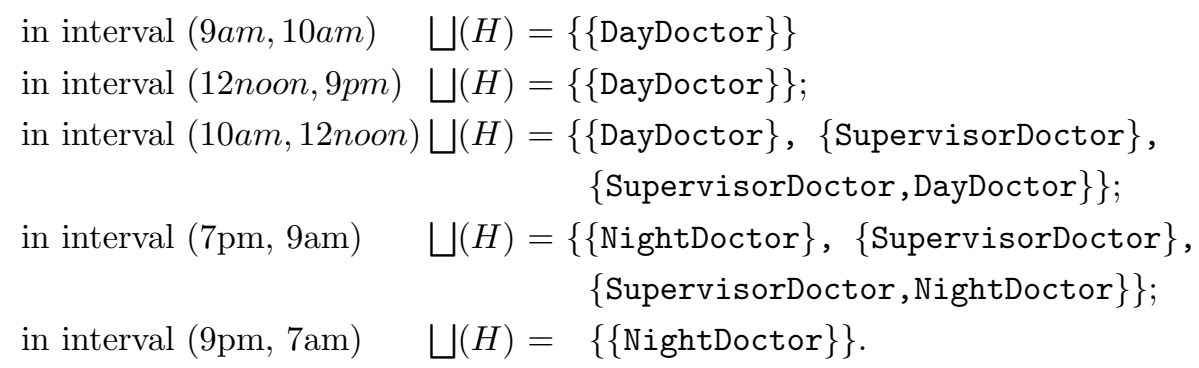

Next, we present a formal basis for characterizing $\bigsqcup(H)$ for a hybrid linear path. We first present the results for a hybrid linear path consisting of only two monotype linear components in the following lemma and then use it to characterize arbitrary hybrid linear paths.

LEMma 4.1. Let $L h=\left(L_{1}, L_{2}\right)$ be a hybrid linear path such that $L_{1}=\left(X_{1},\langle f\rangle\right)$ and $L_{2}=\left(X_{2},\left\langle f_{2}\right\rangle\right)$, where $X=\left\{x_{1}, x_{2}, \ldots, x_{n}\right\}=X_{1} \cup X_{2}$, and $\left\langle f_{1}\right\rangle \neq\left\langle f_{2}\right\rangle$. Then for a user $u$ assigned only to $S_{L_{1}}$, we have:

$$
\sqcup(L h)= \begin{cases}\sqcup\left(L_{1}\right) & \text { if } \geqslant_{i} \in\left\{\left\langle f_{1}\right\rangle,\left\langle f_{2}\right\rangle\right\} \\ \bigsqcup\left(L_{1}^{U}\right) \bigcup \bigsqcup\left(L_{2}\right) \bigcup \bigsqcup\left(L_{1}^{U}\right) \otimes \sqcup\left(L_{2}\right) & \text { if }\left(\left\langle f_{1}\right\rangle,\left\langle f_{2}\right\rangle\right)=\left(\geqslant_{a}, \geqslant\right) \\ \sqcup\left(L_{1}\right) \bigcup \bigsqcup\left(L_{2}^{L}\right) \bigcup \bigsqcup\left(L_{1}\right) \otimes \bigsqcup\left(L_{2}^{L}\right) & \text { if }\left(\left\langle f_{1}\right\rangle,\left\langle f_{2}\right\rangle\right)=\left(\geqslant, \geqslant_{a}\right)\end{cases}
$$

where, $L_{2}^{L}=\operatorname{sub}_{L}\left(L_{2}\right), L_{2}^{U}=\operatorname{sub}_{U}\left(L_{2}\right)$ and $A \otimes B=\{\{x \bigcup y\} \mid x \in A$ and $y$ $\in B\}$.

Note that, in the computation involving $\bigsqcup(L h)$, the components on the right side are disjoint with respect to each other and hence $|\bigsqcup(L h)|$ is simply the sum of the cardinalities of the components on the right side. Theorem 4.2 determines the $\bigsqcup(H)$ for an arbitrary hybrid linear path.

THEOREM 4.2. Let $L h=\left(L_{1}, L H_{2}\right)$ be a hybrid linear path such that $L_{1}=$ $\left(X_{1},\left\langle f_{1}\right\rangle\right), L H_{2}$ is a linear path over $X_{2}$, and $X=X_{1} \bigcup X_{2}$, where $X_{1}$ and $X_{2}$ are role sets. Furthermore, let $L H_{2}=\left(L_{x}, L H^{\prime}\right)$, where $L_{x}=\left(X_{x},\left\langle f_{x}\right\rangle\right)$ over role 
set $X_{x}$ such that $\left\langle f_{x}\right\rangle \neq\left\langle f_{1}\right\rangle$ and LH' is a linear path, possibly empty. Then, we have the following:

1. if $\left\langle f_{1}\right\rangle=\geqslant_{i}$ then $\bigsqcup(L h)=\bigsqcup\left(L_{1}\right)$

2. if $\left\langle f_{1}\right\rangle=\geqslant_{a}$ then

$$
\sqcup(L h)= \begin{cases}\sqcup\left(L_{1}\right) & \text { if }\left(\left\langle f_{x}\right\rangle=\geqslant_{i}\right) \\ \sqcup\left(L_{1}^{U}\right) \bigcup \sqcup\left(L H_{2}\right) \bigcup \sqcup\left(L_{1}^{U}\right) \otimes \sqcup\left(L H_{2}\right) & \text { if }\left(\left\langle f_{x}\right\rangle=\geqslant\right)\end{cases}
$$

3. if $\left\langle f_{1}\right\rangle=\geqslant$ then

$$
\bigsqcup(L h)= \begin{cases}\bigsqcup\left(L_{1}\right) & \text { if }\left(\left\langle f_{x}\right\rangle=\geqslant_{i}\right) \\ \sqcup\left(L_{1}\right) \bigcup \sqcup\left(L H_{2}^{L}\right) \bigcup \sqcup\left(L_{1}\right) \otimes \sqcup\left(L H_{2}^{L}\right) & \text { if }\left(\left\langle f_{x}\right\rangle=\geqslant_{a}\right)\end{cases}
$$

The next example illustrates the use of the above theorem and refers to Fig. 5. We note that to compute $\bigsqcup(H)$ for the hierarchy in case (c), we need to first compute for cases (a) and (b).

Case (a): Here $L_{1}=r_{3} \geqslant r_{2}$, and $L_{2}=r_{2} \geqslant_{a} r_{1}$. Hence, $\left(\left\langle f_{1}\right\rangle,\left\langle f_{2}\right\rangle\right)=\left(\geqslant, \geqslant_{a}\right)$ applies. Therefore, by lemma 4.1, we have,

$$
\begin{aligned}
\sqcup(L h) & =\sqcup\left(L_{1}\right) \bigcup \sqcup\left(L_{2}^{L}\right) \bigcup\left(\bigsqcup\left(L_{1}\right) \otimes \sqcup\left(L_{2}^{L}\right)\right) \\
& =\left\{\left\{r_{2}\right\},\left\{r_{3}\right\}\right\} \bigcup\left\{\left\{r_{1}\right\}\right\} \bigcup\left(\left\{\left\{r_{2}\right\},\left\{r_{3}\right\}\right\} \otimes\left\{\left\{r_{1}\right\}\right\}\right) \\
& =\left\{\left\{r_{1}\right\},\left\{r_{2}\right\},\left\{r_{3}\right\},\left\{\left\{r_{1}\right\},\left\{r_{2}\right\}\right\},\left\{r_{1}, r_{3}\right\}\right\}
\end{aligned}
$$

Case(b): Here $L_{1}=r_{5} \geqslant{ }_{a} r_{4} \geqslant_{a} r_{3}$, and $L H_{2}$ is the hierarchy in (a). Now, we apply Theorem 4.1. As $\left\langle f_{1}\right\rangle=\geqslant_{a}$, case (2) of the theorem applies. Thus,

$$
\begin{aligned}
\sqcup(L h)= & \bigsqcup\left(L_{1}^{U}\right) \bigcup \sqcup\left(L H_{2}\right) \bigcup \sqcup\left(L_{1}^{U}\right) \otimes \sqcup\left(L H_{2}\right) \\
= & \left\{\left\{r_{4}\right\},\left\{r_{5}\right\},\left\{r_{4}, r_{5}\right\}\right\} \bigcup\left\{\left\{r_{1}\right\},\left\{r_{2}\right\},\left\{r_{3}\right\},\left\{r_{1}, r_{2}\right\},\left\{r_{1}, r_{3}\right\}\right\} \bigcup \\
& \left\{\left\{r_{4}\right\},\left\{r_{5}\right\},\left\{r_{4}, r_{5}\right\}\right\} \otimes\left\{\left\{r_{1}\right\},\left\{r_{2}\right\},\left\{r_{3}\right\},\left\{r_{1}, r_{2}\right\},\left\{r_{1}, r_{3}\right\}\right\} \\
= & \left\{\left\{r_{1}\right\},\left\{r_{2}\right\},\left\{r_{3}\right\},\left\{r_{1}, r_{2}\right\},\left\{r_{1}, r_{3}\right\},\left\{r_{4}\right\},\left\{r_{5}\right\},\left\{r_{4}, r_{5}\right\},\right. \\
& \left\{r_{1}, r_{4}\right\},\left\{r_{1}, r_{5}\right\},\left\{r_{1}, r_{4}, r_{5}\right\},\left\{r_{2}, r_{4}\right\},\left\{r_{2}, r_{5}\right\},\left\{r_{2}, r_{4}, r_{5}\right\}, \\
& \left\{r_{3}, r_{4}\right\},\left\{r_{3}, r_{5}\right\},\left\{r_{3}, r_{4}, r_{5}\right\},\left\{r_{1}, r_{2}, r_{4}\right\},\left\{r_{1}, r_{2}, r_{5}\right\}, \\
& \left.\left\{r_{1}, r_{2}, r_{4}, r_{5}\right\},\left\{r_{1}, r_{3}, r_{4}\right\},\left\{r_{1}, r_{3}, r_{5}\right\},\left\{r_{1}, r_{3}, r_{4}, r_{5}\right\}\right\}
\end{aligned}
$$

Case(c): Here $L 1=r_{7} \geqslant r_{6} \geqslant r_{5}$, and $L H_{2}$ is the hierarchy in (a). Again, we apply Theorem 4.1. Computation can be carried out similarly using:

$$
\begin{aligned}
\sqcup(L h)= & \sqcup\left(L_{1}\right) \bigcup \sqcup\left(L H_{2}^{L}\right) \bigcup \sqcup\left(L_{1}\right) \otimes \sqcup\left(L H_{2}^{L}\right) \\
= & \left\{\left\{r_{5}\right\},\left\{r_{6}\right\},\left\{r_{7}\right\}\right\} \bigcup\left\{\left\{r_{1}\right\},\left\{r_{2}\right\},\left\{r_{3}\right\},\left\{r_{1}, r_{2}\right\},\left\{r_{1}, r_{3}\right\},\left\{r_{4}\right\},\right. \\
& \left.\left\{r_{1}, r_{4}\right\},\left\{r_{2}, r_{4}\right\},\left\{r_{3}, r_{4}\right\},\left\{r_{1}, r_{2}, r_{4}\right\},\left\{r_{1}, r_{3}, r_{4}\right\}\right\} \bigcup \\
& \left\{\left\{r_{5}\right\},\left\{r_{6}\right\},\left\{r_{7}\right\}\right\} \otimes\left\{\left\{r_{1}\right\},\left\{r_{2}\right\},\left\{r_{3}\right\},\left\{r_{1}, r_{2}\right\},\left\{r_{1}, r_{3}\right\},\right. \\
& \left.\left\{r_{4}\right\},\left\{r_{1}, r_{4}\right\},\left\{r_{2}, r_{4}\right\},\left\{r_{3}, r_{4}\right\},\left\{r_{1}, r_{2}, r_{4}\right\},\left\{r_{1}, r_{3}, r_{4}\right\}\right\}
\end{aligned}
$$




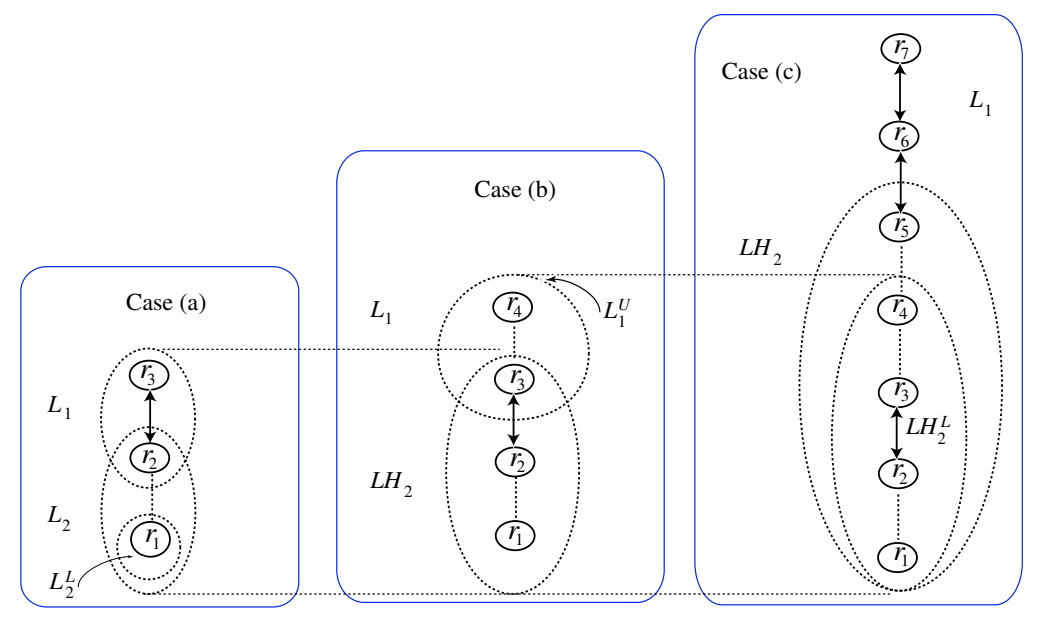

Fig. 5. Computing UAS for a hybrid linear hierarchy

$=\left\{\left\{r_{5}\right\},\left\{r_{6}\right\},\left\{r_{7}\right\},\left\{r_{1}\right\},\left\{r_{2}\right\},\left\{r_{3}\right\},\left\{r_{1}, r_{2}\right\},\left\{r_{1}, r_{3}\right\},\left\{r_{4}\right\}\right.$, $\left\{r_{1}, r_{4}\right\},\left\{r_{2}, r_{4}\right\},\left\{r_{3}, r_{4}\right\},\left\{r_{1}, r_{2}, r_{4}\right\},\left\{r_{1}, r_{3}, r_{4}\right\},\left\{r_{1}, r_{5}\right\}$, $\left\{r_{2}, r_{5}\right\},\left\{r_{3}, r_{5}\right\},\left\{r_{1}, r_{2}, r_{5}\right\},\left\{r_{1}, r_{3}, r_{5}\right\},\left\{r_{4}, r_{5}\right\}$, $\left\{r_{1}, r_{4}, r_{5}\right\},\left\{r_{2}, r_{4}, r_{5}\right\},\left\{r_{3}, r_{4}, r_{5}\right\},\left\{r_{1}, r_{2}, r_{4}, r_{5}\right\}$, $\left\{r_{1}, r_{3}, r_{4}, r_{5}\right\},\left\{r_{1}, r_{6}\right\},\left\{r_{2}, r_{6}\right\},\left\{r_{3}, r_{6}\right\},\left\{r_{1}, r_{2}, r_{6}\right\}$, $\left\{r_{1}, r_{3}, r_{6}\right\},\left\{r_{4}, r_{6}\right\},\left\{r_{1}, r_{4}, r_{6}\right\},\left\{r_{2}, r_{4}, r_{6}\right\},\left\{r_{3}, r_{4}, r_{6}\right\}$, $\left\{r_{1}, r_{2}, r_{4}, r_{6}\right\},\left\{r_{1}, r_{3}, r_{4}, r_{6}\right\},\left\{r_{1}, r_{7}\right\},\left\{r_{2}, r_{7}\right\},\left\{r_{3}, r_{7}\right\}$, $\left\{r_{1}, r_{2}, r_{7}\right\},\left\{r_{1}, r_{3}, r_{7}\right\},\left\{r_{4}, r_{7}\right\},\left\{r_{1}, r_{4}, r_{7}\right\},\left\{r_{2}, r_{4}, r_{7}\right\}$, $\left.\left\{r_{3}, r_{4}, r_{7}\right\},\left\{r_{1}, r_{2}, r_{4}, r_{7}\right\},\left\{r_{1}, r_{3}, r_{4}, r_{7}\right\}\right\}$

A hybrid general hierarchy can have complex inheritance and activation semantics. We note that each hierarchical structure can be broken down into a list of linear paths. We refer to such a decomposition of hierarchy as Linear Path Decomposition of Hybrid Hierarchy (LPDHH). In the following, we consider a general hierarchy rooted at a role and represent it using an LPDHH set of linear components.

Definition 4.3. (Linear Path Decomposition of Hybrid Hierarchy- LPDHH): Let $H=(X,[f])$ be a hierarchy over role set $X$ rooted at role $S_{H}$ with relation set $[f] \subseteq\left\{\geqslant_{i}, \geqslant_{a}, \geqslant\right\}$. We say that $H$ is an ordered set of linear paths (hybrid or monotype), that is, $H=\left(L H_{1}, L H_{2}, \ldots, L H_{m}\right)$ where $L H_{i}$ is a linear path over $X_{i}$, if, for $i, j \in\{1,2, \ldots, m\}, i \neq j$ and $L H_{i}$ is a linear path over $X_{i}$, and the following conditions hold

1. $S_{L H_{i}}=S_{H} ; J_{L H_{i}} \subseteq J_{H}$,

2. $X_{i} \neq X_{j} ; X=\bigcup_{i=1}^{m} X_{i}$ 


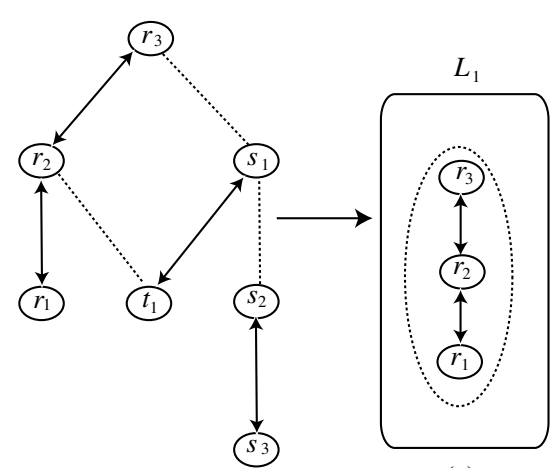

(a)

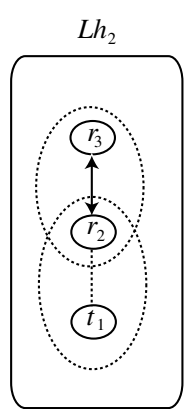

(b)

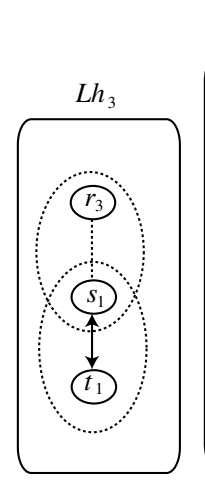

(c)

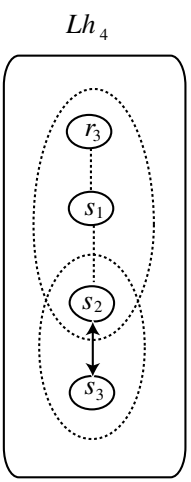

(d)

Fig. 6. Computing UAS for a general hierarchy

3. for all $J \in J_{H}$, there exists no linear path $L H=\left(\left\{S_{H}, x_{\pi_{1}}, x_{\pi_{2}}, \ldots, x_{\pi_{i}}, J\right\}\right.$, $\left.\left[f^{\prime}\right]\right)$, where $\left[f^{\prime}\right] \subseteq[f]$ and $\left\{x_{\pi_{1}}, x_{\pi_{2}}, \ldots, x_{\pi_{i}}\right\} \subseteq X \backslash\left\{S_{H}, J\right\}$, such that $L H \notin\left\{L H_{1}, L H_{2}, \ldots, L H_{m}\right\}$.

We say that $L H_{1}, L H_{2}, \ldots, L H_{m}$ is the complete $L P D H H$ of $H$. $H$ can also be written as $\left(L H_{1}, H^{\prime}\right),\left(H^{\prime \prime}, L H_{m}\right),\left(H_{x}, H_{y}\right)$, etc., each of which is a decomposition of $H$.

Based on the notion of LPDHH of a general hybrid hierarchy, the following theorem shows how we can formally determine $\bigsqcup(H)$ of a general hybrid hierarchy that is not a simple linear path.

TheOREM 4.3. Let $H=(X,[f])=\left(L H_{1}, H_{1}\right)$ be a non-empty and non-linear hierarchy. Then, $\bigsqcup(H)=I \backslash C$, where

$-I=\bigsqcup\left(L H_{1}\right) \bigcup\left(H_{1}\right) \bigcup\left(L H_{1}\right) \backslash B \otimes \bigsqcup\left(H_{1}\right) \backslash B$

$-B=\left(\bigsqcup\left(L H_{1}\right) \sqcap \bigsqcup\left(H_{1}\right)\right)$, where $A \sqcap B=\{S, T \mid S \in A, T \in B$ and $S \cap T \neq \phi\}$

$-C=\{Z \mid Z \in I$, and $\exists x, y \in Z, x \geqslant y\}$.

The theorem determines $\bigsqcup(H)$ of a general hierarchy that has at least one role having multiple juniors, hence making it different from the linear paths. The computation is based on the partitioning of the hierarchy into two components, in which one is a linear component and the other is the remaining part of the hierarchy. This allows us to compute $\bigsqcup(H)$ recursively once we have the linear components. The next example illustrates the working of Theorem 4.3.

Consider the hierarchy in Fig. 6. The linear components of the hierarchy are shown in $(a)-(d)$. Each component's $\bigsqcup(H)$ computed using Theorem 4.3 is shown below, based on which we complete the $\bigsqcup(H)$ of the entire hierarchy. We will write $H_{12}$ to mean the hierarchy formed by components $L_{1}$ and $L h_{2}, H_{13}$ to mean the hierarchy formed by components $L_{1}, L h_{2}$ and $L h_{3}$, and $H_{14}$ to mean the overall hierarchy. First, we get 


$$
\begin{aligned}
\sqcup\left(L_{1}\right)= & \left\{\left\{r_{3}\right\},\left\{r_{2}\right\},\left\{r_{1}\right\}\right\} \\
\sqcup\left(L h_{2}\right)= & \left\{\left\{t_{1}\right\},\left\{r_{2}\right\},\left\{r_{3}\right\},\left\{t_{1}, r_{2}\right\},\left\{t_{1}, r_{3}\right\}\right\} \\
\sqcup\left(L h_{3}\right)= & \left\{\left\{r_{3}\right\},\left\{s_{1}\right\},\left\{t_{1}\right\},\left\{s_{1}, r_{3}\right\},\left\{r_{3}, t_{1}\right\}\right\} \\
\sqcup\left(L h_{4}\right)= & \left\{\left\{r_{3}\right\},\left\{s_{1}\right\},\left\{s_{2}\right\},\left\{s_{3}\right\},\left\{r_{3}, s_{1}\right\},\left\{r_{3}, s_{2}\right\},\left\{r_{3}, s_{3}\right\},\left\{s_{1}, s_{2}\right\},\right. \\
& \left.\left\{s_{1}, s_{3}\right\},\left\{r_{3}, s_{1}, s_{2}\right\},\left\{r_{3}, s_{1}, s_{3}\right\}\right\}
\end{aligned}
$$

Step 1: Consider components $L_{1}$ and $L h_{2}$.

Here,

$$
\begin{aligned}
B & =\bigsqcup\left(L_{1}\right) \sqcap \bigsqcup\left(L h_{2}\right) \\
& =\left\{\left\{r_{3}\right\},\left\{r_{2}\right\},\left\{t_{1}, r_{2}\right\},\left\{t_{1}, r_{3}\right\}\right\} .
\end{aligned}
$$

Therefore, $\quad \sqcup\left(L_{1}\right) \backslash B \otimes \bigsqcup\left(L h_{2}\right) \backslash B=\left\{\left\{r_{1}\right\}\right\} \bigotimes\left\{\left\{t_{1}\right\}\right\}$

$$
=\left\{\left\{r_{1}, t_{1}\right\}\right\} \text {. }
$$

Note that $C$ is empty.

Thus,

$$
\begin{aligned}
& \sqcup\left(H_{12}\right)=I \backslash \mathrm{C}=\mathrm{I} \\
& \quad=\left\{\left\{r_{3}\right\},\left\{r_{2}\right\},\left\{r_{1}\right\},\left\{t_{1}\right\},\left\{r_{1}, t_{1}\right\},\left\{r_{3}, t_{1}\right\},\left\{t_{1}, r_{2}\right\}\right\} .
\end{aligned}
$$

Step 2: Consider component $H_{12}$ (result from Step 1 ) and $L h_{3}$.

$$
\text { Here, } \quad \begin{aligned}
B & =\bigsqcup\left(H_{12}\right) \sqcap \bigsqcup\left(L h_{3}\right) \\
& =\left\{\left\{r_{3}\right\},\left\{t_{1}\right\},\left\{r_{1}, t_{1}\right\},\left\{r_{2}, t_{1}\right\},\left\{r_{3}, t_{1}\right\},\left\{r_{3}, s_{1}\right\}\right\} .
\end{aligned}
$$

Therefore, $\quad \sqcup\left(H_{12}\right) \backslash B \otimes \bigsqcup\left(L h_{3}\right) \backslash B=\left\{\left\{r_{2}\right\},\left\{r_{1}\right\}\right\} \otimes\left\{\left\{s_{1}\right\}\right\}$

$$
\left.=\left\{\left\{r_{1}, s_{1}\right\},\left\{r_{2}, s_{1}\right\}\right\}\right\} \text {. }
$$

Hence, $I=\left\{\left\{r_{3}\right\},\left\{r_{2}\right\},\left\{r_{1}\right\},\left\{t_{1}\right\},\left\{r_{1}, t_{1}\right\},\left\{r_{3}, t_{1}\right\},\left\{t_{1}, r_{2}\right\},\left\{s_{1}\right\}\right.$,

$$
\left.\left\{r_{2}, s_{1}\right\},\left\{r_{1}, s_{1}\right\},\left\{r_{3}, s_{1}\right\}\right\}
$$

Thus, $\bigsqcup\left(H_{13}\right)=I \backslash C$

$$
\begin{aligned}
=\{ & \left\{r_{3}\right\},\left\{r_{2}\right\},\left\{r_{1}\right\},\left\{t_{1}\right\},\left\{r_{1}, t_{1}\right\},\left\{t_{1}, r_{2}\right\},\left\{s_{1}\right\},\left\{r_{2}, s_{1}\right\}, \\
& \left.\left\{r_{1}, s_{1}\right\},\left\{r_{3}, s_{1}\right\}\right\} \text { (C is empty) }
\end{aligned}
$$

Step 3: Consider component $H_{13}$ (result from Step 2) and $L h_{4}$.

Here, $\quad B=\bigsqcup\left(H_{13}\right) \sqcap \bigsqcup\left(L h_{4}\right)$

$$
\begin{aligned}
= & \left\{\left\{r_{3}\right\},\left\{s_{1}\right\},\left\{r_{1}, s_{1}\right\},\left\{r_{2}, s_{1}\right\},\left\{r_{3}, s_{2}\right\},\left\{r_{3}, s_{3}\right\},\left\{s_{1}, s_{2}\right\},\right. \\
& \left.\left\{r_{3}, s_{1}, s_{2}\right\},\left\{r_{3}, s_{1}, s_{3}\right\}\right\}
\end{aligned}
$$

Therefore, $\quad \sqcup\left(H_{13}\right) \backslash B \otimes \sqcup\left(L h_{4}\right) \backslash B$

$$
\begin{aligned}
& =\left\{\left\{r_{2}\right\},\left\{r_{1}\right\},\left\{t_{1}\right\},\left\{r_{1}, t_{1}\right\},\left\{t_{1}, r_{2}\right\}\right\} \otimes\left(\left\{\left\{s_{2}\right\}\right\}\right) \\
& =\left\{\left\{r_{2}, s_{2}\right\}\left\{r_{1}, s_{2}\right\},\left\{t_{1}, s_{2}\right\},\left\{r_{1}, t_{1}, s_{2}\right\},\left\{t_{1}, r_{2}, s_{2}\right\}\right\} .
\end{aligned}
$$

Hence,

$$
\begin{aligned}
\sqcup\left(H_{13}\right)= & I \backslash C \\
= & \left\{\left\{r_{3}\right\},\left\{s_{1}\right\},\left\{s_{2}\right\},\left\{s_{3}\right\},\left\{r_{3}, s_{1}\right\},\left\{r_{3}, s_{2}\right\},\left\{r_{3}, s_{3}\right\},\left\{s_{1}, s_{2}\right\},\right. \\
& \left\{s_{1}, s_{3}\right\},\left\{r_{3}, s_{1}, s_{2}\right\},\left\{r_{3}, s_{1}, s_{3}\right\} \bigcup\left\{\left\{r_{3}\right\},\left\{r_{2}\right\},\left\{r_{1}\right\},\left\{t_{1}\right\},\right. \\
& \left.\left\{r_{1}, t_{1}\right\},\left\{t_{1}, r_{2}\right\},\left\{s_{1}\right\},\left\{r_{2}, s_{1}\right\},\left\{r_{1}, s_{1}\right\},\left\{r_{3}, s_{1}\right\}\right\} \bigcup\left\{r_{2}, s_{2}\right\} \\
& \left.\left\{r_{1}, s_{2}\right\},\left\{t_{1}, s_{2}\right\},\left\{r_{1}, t_{1}, s_{2}\right\},\left\{t_{1}, r_{2}, s_{2}\right\}\right\}
\end{aligned}
$$




$$
\begin{aligned}
= & \left\{r_{3}\right\},\left\{s_{1}\right\},\left\{s_{2}\right\},\left\{s_{3}\right\},\left\{r_{3}, s_{1}\right\},\left\{r_{3}, s_{2}\right\},\left\{r_{3}, s_{3}\right\},\left\{s_{1}, s_{2}\right\}, \\
& \left\{s_{1}, s_{3}\right\},\left\{r_{3}, s_{1}, s_{2}\right\},\left\{r_{3}, s_{1}, s_{3}\right\},\left\{r_{2}\right\},\left\{r_{1}\right\},\left\{t_{1}\right\},\left\{r_{1}, t_{1}\right\}, \\
& \left\{t_{1}, r_{2}\right\},\left\{r_{2}, s_{1}\right\},\left\{r_{1}, s_{1}\right\},\left\{r_{2}, s_{2}\right\}\left\{r_{1}, s_{2}\right\},\left\{t_{1}, s_{2}\right\}, \\
& \left.\left\{r_{1}, t_{1}, s_{2}\right\},\left\{t_{1}, r_{2}, s_{2}\right\}\right\}
\end{aligned}
$$

Based on the theorems, a recursive algorithm can be easily constructed.

\subsection{Acquisition Equivalent Hierarchies}

An important issue is whether or not we can use a hierarchy of one type to achieve what a hierarchy of another type allows. To address such an issue, we need an appropriate notion of equivalence between different hierarchies, as they may be structurally and semantically different. We note that, central to the use of hierarchies in a GTRBAC system is the efficient and fine-grained management of permissions acquired by users assigned to the various roles in the hierarchy. A notion of the equivalence between two hierarchies can be established if we show that the maximum set of permissions that can be acquired by a user in the two hierarchies is the same. The significance of using the maximum set of permissions is that within the equivalent hierarchies, the users can carry the same set of accesses, even though, within each hierarchy, the users may have to activate a different set of roles. Here, we introduce the notion of acquisition-equivalence between two hierarchies. We say that two hierarchies are acquisition-equivalent if they allow the same maximum set of permissions to be acquired by a user assigned to the senior-most role. We use $\underset{\max }{P}(H)$ to refer to the maximum set of permissions that a user can acquire through the senior-most role of the hierarchy $H$ in a session. The notion of acquisition-equivalence is formally defined as follows:

DEFINITION 4.4. (Acquisition equivalence or AC-equivalence of two hierarchies): Let $H_{1}$ and $H_{2}$ be two hierarchies over role set Roles. Then we say that $H_{1}$ and $H_{2}$ are acquisition-equivalent or $A C$-equivalent (written as $H_{1}={ }_{A C} H_{2}$ ), if $\underset{\max }{P}\left(H_{1}\right)=$ $\underset{\max }{P}\left(H_{2}\right)$. Furthermore, $H_{1}={ }_{A C} H_{2}$ and $H_{2}={ }_{A C} H_{3}$, then $H_{1}={ }_{A C} H_{3}$.

The following theorem provides the formal characteristics of an $A C$-equivalent set of hierarchies.

THEOREM 4.4. (AC-equivalent hierarchies $)$ : Let $H_{1}=\left(X,\left[f_{1}\right]\right)=\left(L H_{1}, L H_{2}\right.$ $\left., \ldots, L H_{n}\right)$ and $H_{2}=\left(X,\left\langle f_{2}\right\rangle\right)$ be two hierarchies over the role set $X$. If, for roles $x, y \in X$ and a relation $\langle f\rangle \in\left[f_{1}\right]$, the condition $\left.(x\langle f\rangle y) \in H_{1} \longleftrightarrow\left(x\left\langle f_{2}\right\rangle y\right) \in H_{2}\right)$ holds, then $\mathrm{H}_{1}={ }_{A C} \mathrm{H}_{2}$ (i.e. $\mathrm{H}_{1}$ and $\mathrm{H}_{2}$ are $A C$-equivalent) provided the following holds

-for all $i \in\{1,2, \ldots, n\}$, and for hierarchies $L H^{\prime}, L H_{\text {mid }}, L H^{\prime}$, each possibly empty, the following is satisfied for $\left\langle f_{x}\right\rangle=\geqslant_{i}$ and $\left\langle f_{y}\right\rangle=\geqslant_{a}$

$$
\neg \exists L_{x}, L_{y} \text { such that } L H_{i}=\left(L H^{\prime}, L_{x}, L H_{m i d}, L_{y}, L H^{\prime \prime}\right),
$$

The condition $L H_{i}=\left(L H^{\prime}, L_{x}, L H_{m i d}, L_{y}, L^{\prime \prime}\right)$ implies that in the linear component $\mathrm{LH}_{i}$, there is an $I$-relation that precedes (not necessarily immediately, as $L H_{m i d}$ may not be empty) an $A$-relation. All hierarchies that do not have such a component are $A C$-equivalent to a monotype hierarchy. As a consequence, first, the 
theorem implies that any two monotype hierarchies are AC-equivalent, as the condition $L H_{\text {mid }}=\left(L H^{\prime}, L_{x}, L H_{m i d}, L_{y}, L^{\prime \prime}\right)$ cannot occur in a monotype hierarchy. For example, consider a monotype $I$-hierarchy $H_{1}$. Now construct an $A$-hierarchy $H_{2}$ such that it contains the same roles that are in $H_{1}$, and for each $I$-relation between a pair of roles in $H_{1}, H_{2}$ has an $A$-relation. The theorem indicates that $H_{1}={ }_{A C} H_{2}$. This is because all the permissions that can be used by a user assigned to the senior-most role of $H_{1}$, can also be used by a user assigned to the seniormost role of $H_{2}$. The only difference between the two is that the users may have to activate a different set of roles from the $\bigsqcup(H)$ s of the two hierarchies to do that.

Furthermore, the theorem indicates that every hierarchy that does not contain a linear component shown above is $A C$-equivalent to a monotype hierarchy and hence to each other. This is because if an $I$-relation precedes an A-relation in the hierarchy then the permissions associated with the roles below the A-hierarchy cannot be acquired by any user assigned to the senior-most role, hence, reducing the permissions that can be acquired. The significance of this result is that, in systems where the principle of least privilege is not of much concern, any monotype hierarchy can be used instead of a more complex hybrid hierarchy.

\section{DERIVED RELATIONS IN A HIERARCHY}

In a hierarchy where all the three types of hierarchies can co-exist, a hierarchical relation between a pair of roles that are not directly related may be derived. From the axioms and the hierarchy definitions presented in Section 3, it is easy to see that the three hierarchy types are transitive. For instance, if $(x \geqslant y)$ and $(y \geqslant z)$ then it implies $(x \geqslant z)$. However, in a hybrid hierarchy, the derived relation between an arbitrary pair of roles can have partial transitivity or special hierarchical semantics. For instance, if $\left(x \geqslant_{i} y\right)$ and $(y \geqslant z)$ then it implies $\left(x \geqslant_{i} z\right)$ i.e., transitivity exists only with respect to the permission-inheritance semantics. Similarly, assume $\left(x \geqslant_{a} y\right)$ and $\left(y \geqslant_{i} z\right)$. Here it appears that $x$ and $z$ are not hierarchically related because (1) if a user $u$ assigned to $x$ activates $x$, he does not acquire $z$ 's permissions, and (2) $u$ cannot activate $z$ to acquire its permissions. Note, however, that $u$ can activate $y$ and acquire all the permissions that can be acquired through $z$. We call this special derived type a conditioned derived relation, written as $(x[A](B)\langle f\rangle y)$, and, defined as follows:

Definition 5.1. (Conditioned Derived relation): Let $H$ be a role hierarchy, $x$, $y \in \operatorname{Roles}(H)$ and $A, B \subseteq$ Roles $(H)$. Then $(x[A](B)\langle f\rangle y)$ is called a Conditioned Derived Relation (also read as the derived relation $(x\langle f\rangle y)$ is conditioned on roles in $A$ and $B$ "), if, for all $a \in A$ and $b \in B$, the following holds:

$$
\left(x \geqslant_{a} a\right) \wedge(x\langle f\rangle y) \wedge\left((x=b) \vee\left(x \geqslant_{a} b\right)\right) \wedge\left(b \geqslant_{a} y\right),
$$

where $\langle f\rangle \in\left\{\geqslant_{i}, \geqslant\right\},|A|>0,|B| \geqslant 0$, and $\left(b \geqslant_{a} y\right)$ is a direct relation.

Here, the condition indicates that $x$ is related to each $a \in A$ directly or through a derived $A$-relation, whereas each $a$ is related to $y$ by the $\langle f\rangle$ relation. This implies that a permission that can be acquired through role $y$ can be acquired by a user assigned to role $x$ without activating $y$, but by activating any of the roles in $A$. We note that $B$ may be empty, in which case, the conditioned derived relation is 


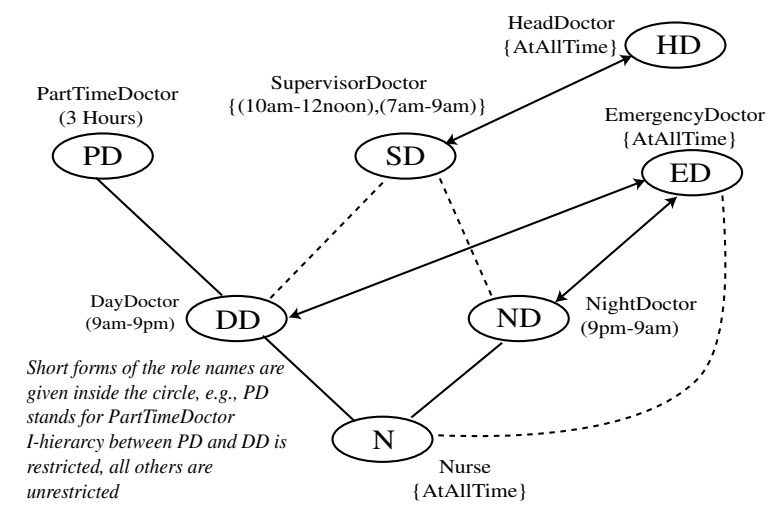

Fig. 7. A hierarchy for a medical department

simply written as $(x[A]\langle f\rangle y)$. If $B$ is not empty then for each $b \in B$, there is an $A$-path from $x$ to $y$ through $b$. If $C=A \cap B$ then, for all $c \in C$, both $(c \geqslant i y)$ and $(c \geqslant a y)$ hold and hence, $(c \geqslant y)$. It is possible that $(x[A](\{x\})\langle f\rangle y)$, which means $(x[A]\langle f\rangle y)$ holds and $\left(x \geqslant_{a} y\right)$ is a direct relation. As we shall see, it is not necessary that each hierarchical path from $x$ to each $a \in A$ contain only $A$-relations; it is only required that a user who is assigned to or can activate $x$ can also activate $a$. This, however, implies that the hierarchical path from $x$ to each $a$ does not contain any $I$-relation as it prohibits activation of a junior role by users assigned to the senior roles. Furthermore, we note that in $(x[A](B)\langle f\rangle y),\langle f\rangle$ is either $\geqslant_{i}$ or $\geqslant$.

Consider the hierarchy of Fig. 7, representing a medical department. PD can be enabled for three hours only. Since it has restricted-inheritance over DD, a user assigned to PD can acquire DD's permissions only in daytime. SD's relation to DD and ND are as discussed in Fig. 7. $\mathrm{N}$ can be $I$-inherited by DD and ND. ED is enabled at all times. The $A$-relation between ED and N allows a user assigned to ED to explicitly act as a nurse besides inheriting N's permissions through DD or ND. Assume that the HD role represents the head doctor of the medical department, which is enabled at all times. HD can act as the supervisor role of doctors, because of the unrestricted relations through SD. Two of the conditioned derived relations are as follows.

(1) $\left(\operatorname{SD}[\{\mathrm{DD}, \mathrm{ND}\}] \geqslant_{i} \mathrm{~N}\right)$ : This is because users assigned to SD can acquire permissions of $\mathrm{N}$ only by activating $\mathrm{DD}$ or ND.

(2) $\left(\mathrm{HD}[\{\mathrm{DD}, \mathrm{ND}, \mathrm{ED}\}](\{\mathrm{ED}\}) \geqslant{ }_{i} \mathrm{~N}\right)$ : This is because users assigned to HD can acquire permissions of $\mathrm{N}$ by activating SD, ND or ED. Furthermore, the users can directly activate N (because of the $A$-path through ED).

\subsection{The Inference Rules}

We now introduce the inference rules that allow derivation of indirect relations between roles from a set of explicitly specified hierarchical relations. Such derived relations can be used to determine the permissions that can be acquired through the activation of a role in a hierarchy by a user. We use $\operatorname{ISen}(y)=\{x \mid(x \geqslant a y)$ is a direct relation $\}$ to denote the set of the immediate $A$-seniors of role $y$. The 
Table V. The inference rules

\begin{tabular}{|c|c|c|}
\hline Rule & Case & Inference Rule \\
\hline \multirow[t]{2}{*}{$R_{1}$} & \multicolumn{2}{|c|}{ (Monotype hierarchy) } \\
\hline & \multicolumn{2}{|c|}{$(x\langle f\rangle y) \wedge(y\langle f\rangle z) \rightarrow(x\langle f\rangle z)$ for all $\langle f\rangle \in\left\{\geqslant_{i}, \geqslant_{a}, \geqslant\right\}$} \\
\hline \multirow{4}{*}{$R_{2}$} & \multicolumn{2}{|c|}{ (Hybrid hierarchy with unconditioned relations) } \\
\hline & 1 & $\begin{array}{l}\left(x\left\langle f_{1}\right\rangle y\right) \wedge\left(y\left\langle f_{2}\right\rangle z\right) \rightarrow\left(x \geqslant_{i} z\right) \text { for all }\left\langle f_{1}\right\rangle,\left\langle f_{2}\right\rangle \in\left\{\geqslant_{i}, \geqslant\right\} \\
\text { such that }\left\langle f_{1}\right\rangle \neq\left\langle f_{2}\right\rangle\end{array}$ \\
\hline & 2 & $(x \geqslant y) \wedge\left(y \geqslant_{a} z\right) \rightarrow\left(x \geqslant_{a} z\right)$ \\
\hline & 3 & $\left(x \geqslant_{a} y\right) \wedge(x\langle f\rangle y) \rightarrow(x[\{y\}]\langle f\rangle z)$ for $\langle f\rangle \in\left\{\geqslant_{i}, \geqslant\right\}$ \\
\hline \multirow{8}{*}{$R_{3}$} & \multicolumn{2}{|c|}{ (Hybrid hierarchy with one unconditioned derived relation) } \\
\hline & \multirow{3}{*}{1} & for $\langle f\rangle \in\left\{\geqslant_{i}, \geqslant a, \geqslant\right\}$ such that $\left\langle f_{1}\right\rangle \neq\left\langle f_{2}\right\rangle$ \\
\hline & & \begin{tabular}{l|l} 
a. & $\left(x[A](B) \geqslant_{i} y\right) \wedge\left(y \geqslant_{i} z\right) \rightarrow\left(x[A \cup C] \geqslant_{i} z\right)$, where \\
& $C=\{y\}$ if $|B|>0$, else $C=\phi$
\end{tabular} \\
\hline & & \begin{tabular}{l|l} 
b. & $\left(x[A](B) \geqslant{ }_{i} y\right) \wedge(y \geqslant z) \rightarrow\left(x[A \cup C](C) \geqslant{ }_{i} z\right)$, where \\
& $C=\{y\}$ if $|B|>0$, else $C=\phi$
\end{tabular} \\
\hline & \multirow{3}{*}{2} & for $\langle f\rangle \in\left\{\geqslant_{i}, \geqslant_{a}, \geqslant\right\}$ such that $\left\langle f_{1}\right\rangle \neq\left\langle f_{2}\right\rangle$ \\
\hline & & $\begin{array}{l}(x[A](B) \geqslant y) \wedge\left(y \geqslant_{i} z\right) \rightarrow\left(x[A \cup C] \geqslant_{i} z\right), \text { where } \\
C=\{y\} \text { if }|B|>0, \text { else } C=\phi\end{array}$ \\
\hline & & b. $\quad(x[A](B) \geqslant y) \wedge(y \geqslant z) \rightarrow(x[A] \geqslant z)$ \\
\hline & \multirow{2}{*}{\multicolumn{2}{|c|}{$\begin{array}{l}3 \\
\text { (Hierarchy with multiple paths between two roles; subscripts } \\
\text { indicate the path number) }\end{array}$}} \\
\hline \multirow{10}{*}{$R_{4}$} & & \\
\hline & 1 & $(x\langle f\rangle y)_{1} \wedge(x\langle f\rangle y)_{2} \rightarrow(x\langle f\rangle y)$ for all $\langle f\rangle \in\left\{\geqslant_{i}, \geqslant_{a}, \geqslant\right\}$ \\
\hline & 2 & $\begin{array}{l}\left(x\left\langle f_{1}\right\rangle y\right)_{1} \wedge\left(x\left\langle f_{2}\right\rangle y\right)_{2} \rightarrow(x \geqslant y) \text { for all }\left\langle f_{1}\right\rangle,\left\langle f_{2}\right\rangle \in\left\{\geqslant{ }_{i}, \geqslant a, \geqslant\right\} \\
\text { such that }\left\langle f_{1}\right\rangle \neq\left\langle f_{2}\right\rangle\end{array}$ \\
\hline & \multirow{4}{*}{3} & for all $\langle f\rangle,\left\langle f_{1}\right\rangle,\left\langle f_{2}\right\rangle \in\left\{\geqslant_{i}, \geqslant\right\}$ such that $\left\langle f_{1}\right\rangle \neq\left\langle f_{2}\right\rangle$ \\
\hline & & \begin{tabular}{l|l} 
a. & $(x[A](B)\langle f\rangle y)_{1} \wedge(x\langle f\rangle y)_{2} \rightarrow(x\langle f\rangle y)$ \\
\end{tabular} \\
\hline & & $(x[A](B)\langle f\rangle y)_{1} \wedge\left(x \geqslant_{a} y\right)_{2} \rightarrow(x[A](\operatorname{ISen}(y))\langle f\rangle y)$ \\
\hline & & \begin{tabular}{l|l} 
c. & $\left(x[A](B)\left\langle f_{1}\right\rangle y\right)_{1} \wedge\left(x\left\langle f_{2}\right\rangle y\right)_{2} \rightarrow(x \geqslant y)$ \\
\end{tabular} \\
\hline & \multirow{3}{*}{4} & for all $\langle f\rangle,\left\langle f_{1}\right\rangle,\left\langle f_{2} \in\left\{\geqslant_{i}, \geqslant\right\}\right.$ such that $\left\langle f_{1}\right\rangle \neq\left\langle f_{2}\right\rangle$ \\
\hline & & \begin{tabular}{l|l} 
a. & $\left(x\left[A_{1}\right]\left(B_{1}\right)\langle f\rangle y\right)_{1} \wedge\left(x\left[A_{2}\right]\left(B_{2}\right)\langle f\rangle y\right)_{2} \rightarrow\left(x\left[A_{1} \cup A_{2}\right]\right.$ \\
& $\left.\left(B_{1} \cup B_{2}\right)\langle f\rangle y\right)$
\end{tabular} \\
\hline & & \begin{tabular}{l|l}
$\mathrm{b.}$ & $\left(x\left[A_{1}\right]\left(B_{1}\right)\left\langle f_{1}\right\rangle y\right)_{1} \wedge\left(x\left[A_{2}\right]\left(B_{2}\right)\left\langle f_{2}\right\rangle y\right)_{2} \rightarrow\left(x\left[A_{1} \cup A_{2}\right]\right.$ \\
& $\left.\left(A \cup B_{1} \cup B_{2}\right) \geqslant_{i} y\right)$ \\
& such that $A=A_{1}$ if $\left\langle f_{1}\right\rangle=\geqslant$ else $A=A_{2}$
\end{tabular} \\
\hline
\end{tabular}

inference rules are as follows.

Definition 5.2. (Inference Rules): Let $H$ be a role hierarchy, $x, y, z \in \operatorname{Roles}(H)$, and $A, A_{1}, A_{2}, B_{1}, B_{2} \subseteq$ Roles $(H)$. Then the inference rules for deriving indirect relations are as shown in Table $V$. 
$R_{1}$ is a trivial case of transitivity using a single hierarchy type. Thus, if $\langle f\rangle$ is $\geqslant_{a}$, then from the two relations $\left(x \geqslant_{a} y\right)$ and $\left(y \geqslant_{a} z\right)$, relation $\left(x \geqslant_{a} z\right)$ is inferred. $R_{2}$ applies to all the pairs with direct or derived relations. This can result in a conditioned derived relation of the form $(x[A]\langle f\rangle z)$. $R_{3}$ deals with each of the cases in which an unconditioned relation follows a conditioned derived relation.

In a hierarchy, there may be more than one relation between a pair of roles. Such a situation arises when there are multiple hierarchical paths between a given pair of roles. $R_{4}$ deals with such cases. Rule $R_{4.1}$ is a trivial case in which both the hierarchical paths are the same unconditioned relation (derived or direct). Rule $R_{4.2}$ captures all the possible combinations of two different unconditioned relations between the same pair. Similarly, rule $R_{4.3}$ captures all the possible combinations of two different hierarchical relations between the same pair of roles in which one is an unconditioned derived relation. Lastly, $R_{4.4}$ captures all the possible combinations of two different hierarchical conditioned derived relations between a pair of roles. Table VI illustrates the application of these rules to determine the derived relations for the hierarchy in Fig. 7 .

Table VI. Application of inference rules over the hierarchy of Fig. 7

\begin{tabular}{|c|c|c|}
\hline \multicolumn{2}{|c|}{ Rule applied } & Derive Relations \\
\hline$R_{1}$ & & $\left(\mathrm{PD} \geqslant{ }_{i} \mathrm{~N}\right),(\mathrm{HD} \geqslant a \mathrm{~N})$ \\
\hline \multirow{5}{*}{$R_{2}$} & 1 & $(\mathrm{ED} \geqslant \mathrm{ND}) \wedge\left(\mathrm{ND} \geqslant{ }_{i} \mathrm{~N}\right)$ implies $\left(\mathrm{ED} \geqslant{ }_{i} \mathrm{~N}\right)$ \\
\hline & 2 & $(\mathrm{HD} \geqslant \mathrm{SD}) \wedge(\mathrm{SD} \geqslant a \quad \mathrm{DD})$ implies $(\mathrm{HD} \geqslant a \quad \mathrm{DD})$ \\
\hline & \multirow{3}{*}{3} & $\left(\mathrm{SD} \geqslant_{a} \mathrm{DD}\right) \wedge\left(\mathrm{DD} \geqslant_{i} \mathrm{~N}\right)$ implies $\left(\mathrm{SD}[\{\mathrm{DD}\}] \geqslant_{i} \mathrm{~N}\right)$ \\
\hline & & $\left(\mathrm{HD} \geqslant{ }_{a} \mathrm{ED}\right) \wedge(\mathrm{ED} \geqslant \mathrm{ND})$ implies $(\mathrm{HD}[\{\mathrm{ED}\}] \geqslant \mathrm{ND})$ \\
\hline & & $(\mathrm{HD} \geqslant a \quad \mathrm{DD}) \wedge(\mathrm{DD} \geqslant \mathrm{N})$ implies $(\mathrm{HD}[\{\mathrm{DD}\}] \geqslant \mathrm{ND})$ \\
\hline \multirow[t]{2}{*}{$R_{3}$} & \multirow[t]{2}{*}{$2 \mathrm{a}$} & $(\mathrm{HD}[\{\mathrm{ED}\}] \geqslant \mathrm{DD}) \wedge\left(\mathrm{DD} \geqslant{ }_{i} \mathrm{~N}\right)$ implies $\left(\mathrm{HD}[\{\mathrm{ED}\}] \geqslant_{i} \mathrm{~N}\right)$ \\
\hline & & $(\mathrm{HD}[\{\mathrm{ED}\}] \geqslant \mathrm{DD}) \wedge\left(\mathrm{ND} \geqslant{ }_{i} \mathrm{~N}\right)$ implies $\left(\mathrm{HD}[\{\mathrm{ED}\}] \geqslant{ }_{i} \mathrm{~N}\right)$ \\
\hline \multirow{5}{*}{$R_{4}$} & 1 & $\left(\mathrm{ED} \geqslant_{i} \mathrm{~N}\right)($ one through DD, another through ND) \\
\hline & 2 & $\left(\mathrm{ED} \geqslant{ }_{i} \mathrm{~N}\right) \wedge\left(\mathrm{ED} \geqslant a_{a} \quad \mathrm{~N}\right)$ implies $(\mathrm{ED} \geqslant \mathrm{N})$ \\
\hline & $3 \mathrm{~b}$ & $\begin{array}{l}\left.\left(\mathrm{HD}[\{\mathrm{ED}\}] \geqslant_{i} \mathrm{~N}\right) \wedge(\mathrm{ED} \geqslant a \mathrm{~N}) \text { implies } \mathrm{HD}[\{\mathrm{ED}\}](\{\mathrm{ED}\}) \geqslant{ }_{i} \mathrm{~N}\right) \\
\text { which is the same as }(\mathrm{HD}[\{\mathrm{ED}\}] \geqslant \mathrm{N})\end{array}$ \\
\hline & $4 \mathrm{a}$ & $\left(\mathrm{HD}[\{\mathrm{DD}\}] \geqslant_{i} \mathrm{~N}\right) \wedge(\mathrm{HD}[\{\mathrm{ND}\}] \geqslant \mathrm{N})$ implies $\left.\mathrm{HD}[\{\mathrm{DD}, \mathrm{ND}\}] \geqslant_{i} \mathrm{~N}\right)$ \\
\hline & $4 \mathrm{~b}$ & $\left(\mathrm{HD}[\{\mathrm{DD}, \mathrm{ND}\}] \geqslant{ }_{i} \mathrm{~N}\right) \wedge(\mathrm{HD}[\{\mathrm{ED}\}] \geqslant \mathrm{N})$ implies $\left.\mathrm{HD}[\{\mathrm{DD}, \mathrm{ND}, \mathrm{ED}\}] \geqslant_{i} \mathrm{~N}\right)$ \\
\hline
\end{tabular}

\subsection{Soundness and Completeness of the Inference Rules}

In this section, we show that the set of inference rules introduced above is sound and complete, using the notion of authorization consistent hierarchies, which is defined below. In the definition, we use predicate can_activate $(u, r, H)$ to mean that $u$ can activate role $r$ in role hierarchy $H$. Similarly, we use predicate can_be_acquired $(p$, $r, H)$ to mean that permission $p$ can be acquired through role $r$ using permissioninheritance semantics in hierarchy $H$. Let $\operatorname{UAH}(H)$ and $\operatorname{PAH}(H)$ be sets of all the user-role and role-permission assignments related to the roles in $\operatorname{Roles}(H)$. 

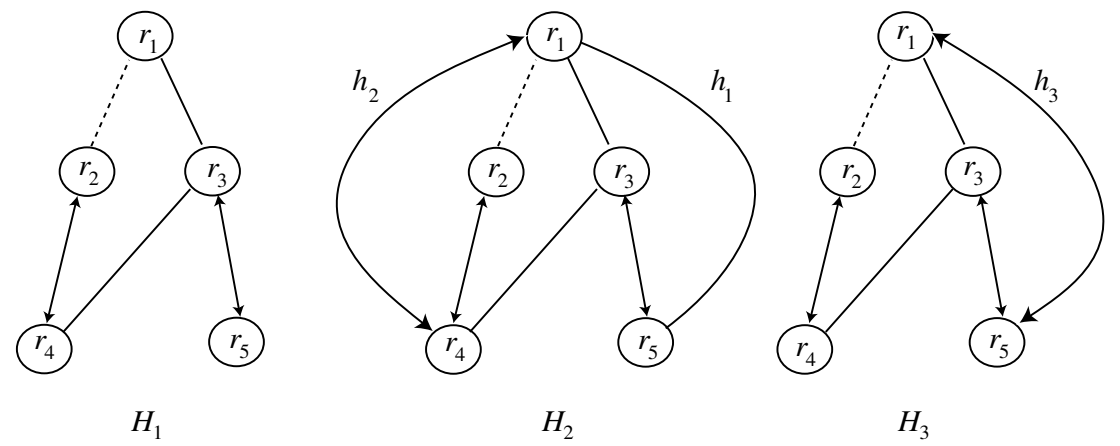

Fig. 8. Example of AC-equivalence; $H_{1} \approx H_{2}, H_{1} \nsim H_{3}$ and $H_{2} \nsim H_{3}$

Definition 5.3. (Authorization consistent hierarchies): Let $\mathrm{H}_{1}$ and $\mathrm{H}_{2}$ be two hierarchies such that $\operatorname{Roles}\left(H_{1}\right)=\operatorname{Roles}\left(H_{2}\right), \operatorname{UAH}\left(H_{1}\right)=\operatorname{UAH}\left(H_{2}\right)$ and $\operatorname{PAH}\left(H_{1}\right)$ $=\operatorname{PAH}\left(H_{2}\right)$. Then, we say that $H_{1}$ and $H_{2}$ are authorization consistent (written as $\left.H_{1} \approx H_{2}\right)$ if for all $r \in \operatorname{Roles}\left(H_{1}\right)$, the following conditions hold:

1. $\forall u \in$ Users, can_activate $\left(u, r, H_{1}\right) \longleftrightarrow$ can_activate $\left(u, r, H_{2}\right)$,

2. $\forall p \in$ Permissions, can_be_acquired $\left(p, r, H_{1}\right) \longleftrightarrow$ can_be_acquired $\left(p, r, H_{2}\right)$.

Here, we note that the two hierarchies considered have the same set of roles, user-role assignments and role-permission assignments. Condition (1) implies that if a user $u$ can activate a role $r$ in $\operatorname{Roles}\left(H_{1}\right)$, then he can activate it even if $H_{1}$ is replaced by $\mathrm{H}_{2}$ (and vice versa). Similarly, the second condition says that the set of permissions that can be acquired through a role under $H_{1}$ is also the same set of permissions that can be acquired through that role in $H_{2}$ for any given user. This signifies that if two hierarchies are authorization consistent then a user assigned to a role can activate exactly the same set of roles and acquire the same set of permissions under the two hierarchies. This means the permission-inheritance and role-activation semantics in the two hierarchies are the same even if the sets of hierarchical relations in the two hierarchies are different. Fig. 8 depicts an example of the notion of authorization consistency. Here, the hierarchy relation $h_{1}$ in $H_{2}$ can be inferred from the hierarchical relations $\left(r_{1} \geqslant_{a} r_{3}\right)$ and $\left(r_{3} \geqslant_{a} r_{5}\right)$, whereas, $h_{2}$ can be inferred from the two hierarchical paths from role $r_{1}$ to $r_{4}$. Hence, $H_{2}$ adds no new access capability compared to $H_{1}$. However, $h_{3}$ in $H_{3}$ cannot be inferred from the hierarchical relations $\left(r_{1} \geqslant{ }_{a} r_{3}\right)$ and $\left(r_{3} \geqslant_{a} r_{5}\right)$. In $H_{3}$, a user assigned to $r_{3}$ can activate $r_{5}$ also, which is not possible in $H_{1}$ or $H_{2}$. Hence, $\left(H_{1} \nsim H_{3}\right)$, and $\left(H_{2} \nsim H_{3}\right)$. We use this notion of authorization consistency between two hierarchies to show that the set of rules presented above is sound, i.e., each new derived relation that can be deduced from a given set of hierarchical relations using the rules produces the same inheritance and activation semantics that is already present in the original hierarchy. Within a hierarchy $H$, we use $h_{x z}$ to represent $(x\langle f\rangle z)$ for $\langle f\rangle \in\left\{\geqslant_{i}, \geqslant_{a}, \geqslant\right\}$ or $(x[A](B)\langle f\rangle z)$ for $\langle f\rangle \in\left\{\geqslant_{i}, \geqslant_{a}\right\}$, where $x, z \in$ Roles $(H)$ and $A, B \subseteq \operatorname{Roles}(H)$. The following theorem formally states this result.

THEOREM 5.1. (Soundness of rules $R_{1}-R_{4}$ ): Given a role hierarchy $H$, if a new hierarchical relation $h_{x z}$ is derived from hierarchical relations in $H$ as per 
rules $R_{1}-R_{4}$, and $H^{\prime}=H \cup\left\{h_{x z}\right\}$, then $H$ and $H^{\prime}$ are authorization consistent, i.e. $H \approx H^{\prime}$.

The theorem implies that the new relations derived using the rules do not allow a user to inherit more (or less) permissions than was allowed to him before the derived relation is added. Similarly, the new derived relation does not allow a user to be able to activate more (or less) number of roles than that was allowed before the derived relation is introduced. Next, we present the completeness theorem for the rules $R_{1}-R_{4}$. We write $H\left[R_{1}-R_{4}\right] \vDash h_{x, z}$ to indicate that the relations in $H$ can logically derive relation $h_{x, z}$ using rules $R_{1}-R_{4}$.

THEOREM 5.2. (Completeness of rules $R_{1}-R_{4}$ ): Given a role hierarchy $H$, rules $R_{1}-R_{4}$ are complete; That is, if $\neg H\left[R_{1}-R_{4}\right] \vDash h_{x, z}$, for any pair of roles $x, z \in \operatorname{Roles}(H)$, then $H \nsim H \cup\left\{h_{x, z}\right\}$, i.e., the hierarchies $H$ and $H^{\prime}=H \cup\left\{h_{x, z}\right\}$ are not authorization consistent.

The theorem indicates that if a relation, say $\langle f\rangle$, between any two roles, say $x$ and $z$, of Roles $(H)$ cannot be derived from the hierarchical relations in $H$, then any role hierarchy containing such a relation is not authorization consistent with $H$. In other words, we can take every pair of roles $(x, z)$ of Roles $(H)$ and every possible hierarchical relations between them, including conditioned derived relations and extend $H$ by adding it to get $H^{\prime}$. If we get $H \approx H^{\prime}$, the theorem implies that the rules $R_{1}-R_{4}$ will derive it. Hence, this shows that the rules are complete. Using the transitivity of the hierarchical relations and considering all the cases of the rules, we can easily construct the proofs. The proofs for both the theorems are provided in Appendix B.

\section{HIERARCHY TRANSFORMATION ALGORITHMS}

In an organization, roles evolve with time, affecting the existing role hierarchies. New roles may need to be added and old ones deleted or modified. Permission sets of existing roles or their temporal properties may need to be altered. Making such changes may require restructuring the hierarchies to avoid undesirable situations. In this section, we analyze transformations of a role hierarchy when a role is added, modified, or deleted that best maintain the permission inheritance and role activation semantics of the original hierarchy.

\subsection{Role Addition}

Typically, a new role is added to an existing hierarchy to distribute a set of new permissions among the already existing roles in the hierarchy. Before we add a new role to a hierarchy, we need to properly identify the existing sets of roles that can be its seniors and juniors based on the permission distribution requirements. Furthermore, we need to consider the existing constraints on and/or among roles in the hierarchy to determine possible new relations between the existing roles and the new role. While pre-existing hierarchical semantics may need to be maintained, the permission acquisition and role activation semantics of the original hierarchy may need to be relaxed to allow some desirable changes.

Let $r_{n}$ be the new role to be added in the original hierarchy $H_{o}$. Suppose $r_{n}$ is to be added between roles $s$ and $j$, and $(s\langle f\rangle j) \in H_{o}$. By adding the new role, assume 
we obtain the new hierarchy $H_{n}$. That is, $H_{n}=\left(H_{o} \cup\left\{\left(s\left\langle f_{1}\right\rangle r_{n}\right),\left(r_{n}\left\langle f_{2}\right\rangle j\right)\right\}\right) \backslash(s\langle f\rangle j)$ for some hierarchy relations $\left\langle f_{1}\right\rangle$ and $\left\langle f_{2}\right\rangle$.

In general, when a new role is added, we require that the original permission acquisition and/or role activation semantics of the hierarchy is maintained. These semantic requirements can be represented as the conditional criteria shown in Table VII that should be valid after the transformation has been made.

Table VII. Criteria for hierarchy transformations

\begin{tabular}{|c|c|c|}
\hline \multicolumn{3}{|r|}{ Criteria } \\
\hline 1 & $\mathrm{C} 1$ & $\begin{array}{c}\forall u \in \text { Users, } r \in \operatorname{Roles}\left(H_{o}\right) \\
\text { can_activate }\left(u, r, t, H_{o}\right) \leftrightarrow \text { can_activate }\left(u, r, t, H_{n}\right)\end{array}$ \\
\hline & $\mathrm{C} 2$ & $\begin{array}{c}\forall p \in P\left(\operatorname{Roles}\left(H_{o}\right)\right), r \in \operatorname{Roles}\left(H_{o}\right) \\
\text { can_be_acquired }\left(p, r, t, H_{o}\right) \leftrightarrow \text { can_be_acquired }\left(p, r, t, H_{n}\right)\end{array}$ \\
\hline 2 & $C 2^{r}$ & $\begin{array}{c}\forall L H=\left(\left\{x_{1}, x_{2}, \ldots, x_{i} s, j\right\},\left[f_{L H}\right]\right), r \in\left\{x_{1}, x_{2}, \ldots, x_{i}, s\right\}, \\
(\mathrm{r}\langle\mathrm{f}\rangle \mathrm{j}) \in H_{o} \leftrightarrow\left(\mathrm{r}\left[r_{n}\right]\langle\mathrm{f}\rangle \mathrm{j}\right) \in H_{n}, \text { where }\left\{x_{1}, x_{2}, \ldots, x_{i} s, j\right\} \subseteq \operatorname{Roles}\left(H_{o}\right), \\
{\left[f_{L H}\right] \subseteq\left\{\geqslant_{i}, \geqslant a, \geqslant\right\} \text { and }\langle f\rangle \in\left\{\geqslant_{i}, \geqslant_{a}\right\}}\end{array}$ \\
\hline
\end{tabular}

Table VIII. Scenarios for hierarchy transformations

\begin{tabular}{|c|c|}
\hline \multicolumn{2}{|c|}{ Scenarios for role addition } \\
\hline S1 & No extra constraint is added with respect to the new role $r_{n} ;$ \\
\hline S2 & A permission-centric activation constraint is added for the new role $r_{n}$ \\
\hline S3 & A user-centric activation constraint is added for the new role $r_{n} ;$ \\
\hline S4 & $\left(s, r_{n}\right)$ is considered to be in DSoD \\
\hline S5 & $\left(r_{n}, j\right)$ is considered to be in DSoD \\
\hline
\end{tabular}

Criteria $\mathrm{C} 1$ states that a role of the original hierarchy $H_{o}$ can be activated by a user in the new hierarchy $H_{n}$ if and only if the user can activate it in $H_{o}$. Similarly, criteria $\mathrm{C} 2$ states that the permissions associated with the roles of the original hierarchy $H_{o}$ can be acquired by a user in the new hierarchy $H_{n}$ if and only if the user can acquire it in $H_{o}$. Ideally both $\mathrm{C} 1$ and $\mathrm{C} 2$ should apply after the transformation is made as they imply that the permission acquisition and role activation semantics of $H_{o}$ are not changed. However, it may not be possible to retain the original semantics. In such a situation, the default case may be to not allow the role to be added at all. That is, however, too restrictive. Another option is to try to retain partial semantics, i.e., we satisfy either criteria $\mathrm{C} 1$ or $\mathrm{C} 2$. It is also possible to accommodate the new role in the existing hierarchy in such a way that original acquisition and activation relations are replaced by the conditioned derived relations between pre-existing roles. For example, assume that in $H_{o}$ the relation between $s$ and $j$ is $(s\langle f\rangle j)$, where $\langle f\rangle \in\left\{\geqslant_{i}, \geqslant\right\}$, and that it is required to add the new role $r_{n}$ so that $\left(s \geqslant{ }_{a} r_{n}\right)$ and $\left(r_{n} \geqslant_{i} j\right)$. If we replace $(s\langle f\rangle j)$ by these two relations, the original semantics is lost, but it does not mean that users assigned to $s$ cannot acquire $j$ 's permissions. Hence, the original semantics is completely lost. Such cases allow flexible transformations where the original semantics cannot 
be completely retained. Hence, we say that such a scenario results in a restricted transformation. We represent such a scenario as criteria $\mathrm{C} 2^{r}$ indicating that criteria $\mathrm{C} 2$ has been satisfied in a restricted sense.

When a new role is added, various new constraints related to the new role may need to be added as well. It is important to note that the above criteria may not be satisfied if we introduce new constraints along with the new role. We consider the five scenarios (S1 through S5), shown in Table VIII to describe the addition of constraints related to the new role and describe with regards to them various transformations that satisfy criteria $\mathrm{C} 1, \mathrm{C} 2$ and/or $\mathrm{C} 2^{r}$, as shown in Table IX. Here, $\sqrt{ }$ indicates that the transformation satisfies the indicated criteria under the given scenario and $x$ indicates that the criterion is not satisfied. Note that the static separation of duty (SSoD) constraint between hierarchically related roles is not appropriate [Gavrila and Barkley 1998]. However, an A-hierarchy allows dynamic SoD (DSoD) to be defined on a role [Joshi et al. 2002]. Note that DSoD restricts a user from activating conflicting roles simultaneously. Hence, we only consider DSoD between roles as a scenario.

\begin{tabular}{|c|c|c|c|c|c|c|c|c|c|}
\hline & & $\begin{array}{c}(s\langle f\rangle j) \\
\in H_{o}\end{array}$ & $\left(s\left\langle f_{1}\right\rangle r_{n}\right),\left(r_{n}\left\langle f_{2}\right\rangle j\right) \in H_{n}$ & $\begin{array}{l}\text { Criteria } \\
\text { Satisfied }\end{array}$ & S1 & S2 & S3 & S4 & S5 \\
\hline \multirow{3}{*}{$a$} & $i$ & \multirow{3}{*}{$(s \geqslant a j)$} & $\left(s \geqslant a r_{n}\right),\left(r_{n} \geqslant a j\right)$ & \multirow{3}{*}{$\mathrm{C} 1, \mathrm{C} 2$} & $\sqrt{ }$ & $\sqrt{ }$ & $x$ & $\sqrt{ }$ & $\sqrt{ }$ \\
\hline & $i i$ & & $\left(s \geqslant r_{n}\right),\left(r_{n} \geqslant_{a} j\right)$ & & $\sqrt{ }$ & $\times$ & $\sqrt{ }$ & $\times$ & $\sqrt{ }$ \\
\hline & $i i i$ & & $\left(s\langle f\rangle r_{n}\right),\left(r_{n} \geqslant_{i} j\right)$, for any $\langle f\rangle$ & & $x$ & $x$ & $x$ & $\times$ & $x$ \\
\hline \multirow{3}{*}{$b$} & $i$ & \multirow{3}{*}{$\left(s \geqslant_{i} j\right)$} & $\left(s \geqslant_{a} r_{n}\right),\left(r_{n} \geqslant_{i} j\right)$ & $\mathrm{C} 1, \mathrm{C} 2^{r}$ & $\sqrt{ }$ & $\sqrt{ }$ & $\times$ & $\sqrt{ }$ & $x$ \\
\hline & $i i$ & & $\begin{array}{l}\left(s \geqslant{ }_{i} r_{n}\right),\left(r_{n} \geqslant_{i} j\right) \\
\left(s \geqslant r_{n}\right),\left(r_{n} \geqslant i j\right) \\
\left(s \geqslant i r_{n}\right),\left(r_{n} \geqslant j\right)\end{array}$ & $\mathrm{C} 1, \mathrm{C} 2$ & $\sqrt{ }$ & $\times$ & $\sqrt{ }$ & $\times$ & $\times$ \\
\hline & $i i i$ & & $\begin{array}{c}\left(s \geqslant r_{n}\right),\left(r_{n} \geqslant j\right) \\
\left(s \geqslant a r_{n}\right),\left(r_{n} \geqslant i j\right) \\
\left(s\langle f\rangle r_{n}\right),\left(r_{n} \geqslant a j\right) \text { for any }\langle f\rangle\end{array}$ & $\mathrm{C} 1, \mathrm{C} 2$ & $\times$ & $\times$ & $x$ & $\times$ & $x$ \\
\hline \multirow{3}{*}{$c$} & $i$ & \multirow{3}{*}{$(s \geqslant j)$} & $\left(s \geqslant a r_{n}\right),\left(r_{n} \geqslant i\right)$ & $\mathrm{C} 1, \mathrm{C} 2^{r}$ & $\sqrt{ }$ & $\sqrt{ }$ & $x$ & $\sqrt{ }$ & $x$ \\
\hline & $i i$ & & $\left(s \geqslant r_{n}\right),\left(r_{n} \geqslant j\right)$ & \multirow[b]{2}{*}{$\mathrm{C} 1, \mathrm{C} 2$} & $\sqrt{ }$ & $\times$ & $\sqrt{ }$ & $\times$ & $\times$ \\
\hline & $i i i$ & & $\begin{array}{c}\left(s\langle f\rangle r_{n}\right),\left(r_{n} \geqslant j\right) \\
\left(s\langle f\rangle r_{n}\right),\left(r_{n} \geqslant i j\right), \text { or } \\
\left(s\langle f\rangle r_{n}\right),\left(r_{n} \geqslant a j\right), \text { for any }\langle f\rangle\end{array}$ & & $\times$ & $\times$ & $x$ & $\times$ & $\times$ \\
\hline
\end{tabular}

Fig. 9 illustrates various transformation cases and Table IX shows these transformations against various scenarios listed in Table VIII. They can be easily explained by applying the inference rules to infer the derived relation between $s$ and $j$ in $H_{n}$. Note that DSoD constraints are allowed among roles that are only $A$-hierarchically related [Joshi et al. 2002]. Similarly, permission-centric activation constraints are appropriate when $A$-hierarchy is used whereas user-centric activation constraint is appropriate in an $I$ or $I A$-hierarchy [Joshi et al. 2002]. 


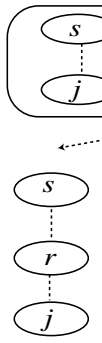

(i)

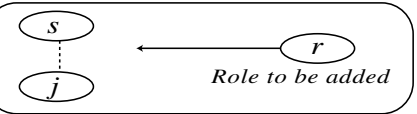

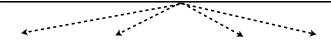

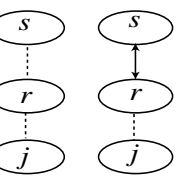

(ii)

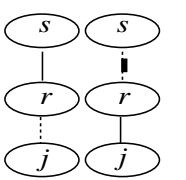

$\stackrel{\text { (iii) }}{>}$

(a)

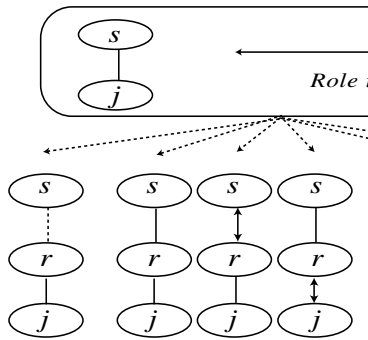

(ii)

(b)

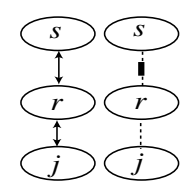

(iii)

$\stackrel{<}{>}$

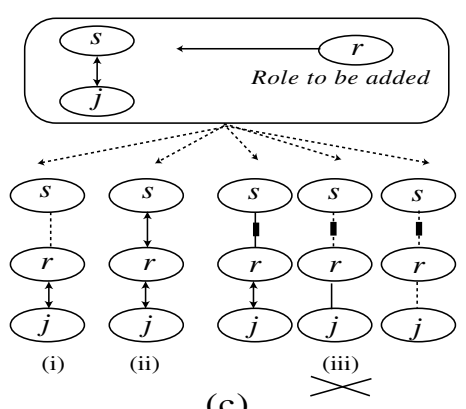

(c)

Fig. 9. Addition of a new role $r$ between roles $s$ and $j$

Fig. $9(a)$ depicts the addition of role $r_{n}$ when $(s \geqslant a j)$ for case $(a)$ of Table VIII. As Fig. 9(a) shows, only cases $(i)$ and $(i i)$ satisfy $\mathrm{C} 1$ and $\mathrm{C} 2$ under some scenarios. Case $(i)$ allows defining a permission-centric activation time constraint on role $r_{n}$ (scenario S2) because of the new relation $\left(s \geqslant{ }_{a} r_{n}\right)$. Defining a DSoD constraint between roles $s$ and $r_{n}$, and $r$ and $j$ (scenarios $\mathrm{S} 4$ and $\mathrm{S} 5$ ) is allowed by the $A$ relations between them. Case $(i i)$ allows a user-centric activation-time constraint on role $r_{n}$ because of the relations $\left(s \geqslant r_{n}\right),\left(r_{n} \geqslant a j\right)$. Cases depicted in (iii) do not retain original hierarchical properties, not even when no constraint is added for the new role (scenario S1). The main reason is the introduction of an $I$-relation that removes the original activation semantics. Note that, in cases depicted in (iii), if we retain original relation $\left(s \geqslant_{a} j\right)$ instead of replacing with new ones as we have discussed, the original semantics will be intact. Retaining $\left(s \geqslant_{a} j\right)$ in cases $(i)$ and (ii) would result in redundant relations between $s$ and $j$.

Fig. 9(b) depicts the addition of role $r_{n}$ when $\left(s \geqslant_{i} j\right)$ is in $H_{o}$. Because of the new relation $\left(s \geqslant{ }_{a} r_{n}\right)$ for case $(i)$, we see that $\mathrm{C} 2^{r}$ is satisfied for some scenarios as $s$ and $j$ are related by an $I$-relation in $H_{o}$. Furthermore, $\left(s \geqslant_{a} r_{n}\right)$ allows the permission-centric activation constraints on $r_{n}$ and DSoD constraints on $s$ and $r_{n}$ (scenarios S4 and S5). Cases depicted in Fig. 9(b)(ii) maintain the original derived relation $\left(s \geqslant_{i} j\right)$. These choices allow defining a user-centric activation time constraint on role $r_{n}$ (scenario S3). Cases depicted in Fig. 9(b)(iii) either introduce an $A$-relation between $s$ and $j$, which is not present in original hierarchy, or make 
them hierarchically unrelated, removing the original $I$-relation between $s$ and $j$. Hence, such transformations do not satisfy any criteria. As before, we could opt to retain $\left(s \geqslant_{i} j\right)$. This would be useful in case $(i)$. In cases depicted in Fig. 9(b)(ii), it would be redundant. In cases shown in Fig. 9(b) (iii), it would be useful when there is an $I$-relation between $s$ and $r_{n}$ and an $A$-relation between $r_{n}$ and $j$.

Fig. $9(c)$ depicts the addition of role $r_{n}$ when $(s \geqslant j)$ is in $H_{o}$. Case $(i)$ introduces an $A$-relation between $s$ and $r_{n}$, removing the $I$-relation between $s$ and $j$; hence, only $\mathrm{C} 2^{r}$ can be satisfied for some scenarios. Case $(i i)$ retains the original relation between $s$ and $j$. However, there is more restriction on Case $(i i)$ in terms of what constraints can be defined for $r_{n}$ than in Case (i). In cases depicted in (iii), the $I$-relation between $s$ and $j$ is completely removed, hence the transformations do not satisfy any criteria. As in the other cases, we can retain the original relation $(s \geqslant j)$. This is useful for all but case (ii), where it would be redundant. This is because, in all other cases, only partial semantics can be retained by replacing the relation by the two new relations. It is important to note that, in all three cases discussed above, retaining the original $(s\langle f\rangle j)$ will provide less flexibility in terms of the scenarios that we have considered.

\subsection{Role Deletion}

When a role is deleted from a hierarchy, the crucial issue is what to do with the permissions associated with it and the users assigned to it. Generally, it will be required that the permissions be retained in the system, and make them available through other roles in the hierarchy. This requires redistributing the permissions associated with the deleted role to other roles in the hierarchy, and reassigning the users originally assigned to the deleted role. We identify the following three approaches for the deletion of a role from a hierarchy with respect to the privilege distribution: (1) the first approach is to reassign the permissions of the deleted role to its immediate seniors; (2) the second approach is to reassign the permissions of the deleted roles to its immediate juniors; and (3) the third approach is to reassign the permissions of the deleted role to each of the senior roles through which the permissions of the deleted role can be acquired within the original hierarchy.

One key problem with these approaches is the reassignment of the users who were originally assigned to the deleted role. As users assigned to the deleted roles need to be reassigned to junior roles or the senior roles, any reassignment will result in either a privilege escalation or privilege depletion of some users assigned to the roles in the hierarchy. The third approach is ad-hoc in nature and inefficient as permissions are explicitly assigned to all senior roles through which they could be acquired before the transformation. Hence, it defeats the purpose of a hierarchy structure. In practice, this approach may be applicable when the whole hierarchy needs to be restructured. We do not discuss the third approach further.

As before, let $H_{o}$ be the original hierarchy and $H_{n}$ the new hierarchy obtained by deleting role $r$. Furthermore, let $U_{r}$ and $P_{r}$ be the sets of users and permissions explicitly assigned to role $r$. For each immediate junior $j$ of $r$, let $U_{j}$ be the set of users assigned to $j$. Let $s$ be an immediate senior of $r$. Table $\mathrm{X}$ depicts different cases of transformations for the first and the second approaches that attempt to meet the criteria $\mathrm{C} 1$ and $\mathrm{C} 2$ introduced earlier.

As shown in the table, for both approaches, it is possible that the users are 
Table X. Deletion of a role using the first and second approach

\begin{tabular}{|c|c|c|c|c|}
\hline & \multicolumn{2}{|c|}{ The First Approach } & \multicolumn{2}{|c|}{ The Second Approach } \\
\hline $\begin{array}{c}\text { For }(r\langle f\rangle j) \in H_{o}, \\
\langle f\rangle \text { is }\end{array}$ & $\begin{array}{c}\text { For }(s\langle h\rangle r) \in H_{o}, \\
\langle h\rangle \text { is }\end{array}$ & $\begin{array}{c}\text { For }(s\langle f\rangle j) \in H_{n}, \\
\langle f\rangle \text { is } \\
\text { (for appropriate } \\
\text { transformation) }\end{array}$ & $\begin{array}{c}\text { For }(s\langle f\rangle r) \in H_{o}, \\
\langle h\rangle \text { is }\end{array}$ & $\begin{array}{c}\text { For }(s\langle f\rangle j) \in H_{n}, \\
\langle f\rangle \text { is } \\
\text { (for appropriate } \\
\text { transformation) } \\
\end{array}$ \\
\hline \multirow{3}{*}{$A$-hierarchy $(\geqslant a)$} & $I$-hierarchy $\left(\geqslant_{i}\right)$ & no relation & $I$-hierarchy $\left(\geqslant_{i}\right)$ & none appropriate \\
\hline & $A$-hierarchy $(\geqslant a)$ & $A$-hierarchy $(\geqslant a)$ & $A$-hierarchy $(\geqslant a)$ & $A$-hierarchy $(\geqslant a)$ \\
\hline & $I A$-hierarchy $(\geqslant)$ & $A$-hierarchy $(\geqslant a)$ & $I A$-hierarchy $(\geqslant)$ & $\begin{array}{c}A \text {-hierarchy }(\geqslant) \\
\text { (restrictive) }\end{array}$ \\
\hline$I$-hierarchy $\left(\geqslant_{i}\right)$ & any & $I$-hierarchy $\left(\geqslant_{i}\right)$ & any & $I$-hierarchy $\left(\geqslant_{i}\right)$ \\
\hline$I A$-hierarchy $(\geqslant)$ & for any $\langle h\rangle$ & $\langle h\rangle$ & for any $\langle h\rangle$ & $\langle h\rangle$ \\
\hline \multirow[t]{2}{*}{ Reassignments $\rightarrow$} & \multicolumn{2}{|c|}{$P_{r}$ is assigned to role $s$} & \multicolumn{2}{|c|}{$P_{r}$ is assigned to role $j$} \\
\hline & $U_{r}$ is assigned to role $s$ & $U_{r}$ is assigned to role $j$ & $U_{r}$ is assigned to role $s$ & $U_{r}$ is assigned to role $j$ \\
\hline $\begin{array}{c}\text { Result of } \\
\text { reassignment } \rightarrow\end{array}$ & $\begin{array}{l}\text { Privilege escalation } \\
\text { for users in } U_{r}\end{array}$ & $\begin{array}{l}\text { Privilege depletion } \\
\text { for users in } U_{r}\end{array}$ & $\begin{array}{c}\text { Privilege escalation } \\
\text { for users in both } U_{r} \text { and } U_{j}\end{array}$ & $\begin{array}{l}\text { Privilege depletion } \\
\text { for users in } U_{j}\end{array}$ \\
\hline
\end{tabular}


reassigned to senior or the junior roles. Privilege escalation of users in $U_{r}$ occurs in the first approach if they are re-assigned to senior roles, privilege depletion occurs if the users in $U_{r}$ are re-assigned to the junior roles. In practice, a choice can be made based on the risk factor related to the privilege escalation and privilege depletion resulting from re-assignment of $U_{r}$. Note that $U_{s}$ and $U_{j}$ are not affected in this case. In the second approach, if the users in $U_{r}$ are reassigned to $s$, privilege escalation similar to that in the first approach occurs with respect to $U_{r}$. In the second approach, privilege escalation also occurs with respect to the users in $U_{j}$. The table further shows what the appropriate transformations are for different sets of relations between $s$ and $r$, and $r$ and $j$ in $H_{o}$.

Fig. 10 depicts various transformations when $\left(r \geqslant_{a} j\right) \in H_{o}$ under the first approach. Note that $s$ may be related to its immediate senior by any of the three hierarchical relations. To show the overall picture, we include roles $x, y$ and $z$ as seniors of $s$ with respect to $I, A$ and $I A$-relations, respectively. Let $\langle h\rangle$ be the original relation between $s$ and $r$. When $\langle h\rangle$ is an $I$-hierarchy, $s$ and $j$ are not hierarchically related, as $s$ does not inherit $j$ 's permissions, neither is any user assigned to $s$ or its seniors able to activate $j$ in $H_{o}$. Hence, case $(i)$ in Fig. 10(a) (i.e. " no relation" between $s$ and $j$ ) retains the original derived relation between $s$ and $j$, (as indicated in the table). The choices (ii), (iii) and (iv) in Fig. 10(a) result in undesirable situations as each one makes something possible that was not originally possible. Similarly, when $\langle h\rangle$ is an $A$ or $I A$-hierarchy, $s$ and $j$ have a derived relation $\left(s \geqslant_{a} j\right)$. Hence, as shown in figures 10(b) and 10(c), after the deletion of role $r$, we can introduce the direct relation $\left(s \geqslant_{i} j\right)$ or $\left(s \geqslant_{a} j\right)$. We note that after the deletion of role $r$, if we have $(s \geqslant j)$, it makes the inheritance of $j^{\prime} s$ permissions by $s$ possible, which was not originally allowed.

The cases for $\left(r \geqslant_{i} j\right)$ or $(r \geqslant j)$ in $H_{o}$ can be similarly explained. When $\left(r \geqslant_{i} j\right)$ $\in H_{o}$, for all relations between $s$ and $r$, the resulting relation between $s$ and $j$ will be $\left(s \geqslant_{i} j\right)$ as shown in the table. It is straightforward to see that it is so when $\langle h\rangle$ is an $I$-relation. If $\langle h\rangle$ is an $I A$-relation, then $\left(s \geqslant_{i} j\right)$ is the derived relation in $H_{o}$ and hence after the transformation, the relation is maintained. However, if $\langle h\rangle$ is an $A$-relation, then the original relation between $s$ and $j$ would be $(s[\{r\}] \geqslant i j)$. If in the transformed hierarchy, we use relation $(s \geqslant i j)$ then users who can activate $s$ cannot activate $j$, but still can acquire $j$ 's permission by activating $s$ in place of the deleted role $r$. Hence, the semantics about a user not being able to activate it but being able to acquire its permissions by activating some senior role is still present in the hierarchy with the new relation $\left(s \geqslant_{i} j\right)$. It is, however, to be noted that this transformation affects the original relations between $j$ and role $s$ or those above it. The change is in terms of what needs be activated to acquire $j$ 's permissions.

Various cases for the second approach can be similarly explained. The key difference is when $\left(r \geqslant_{a} j\right) \in H_{o}$. Here, if $\left(s \geqslant_{i} r\right) \in H_{o}$, no hierarchical relation between $s$ and $j$ can be derived in $H_{o}$; hence, we cannot have any relation between $s$ and $j$. However, no relation between $s$ and $j$ means that the permission set $P_{r}$, now assigned to $j$, cannot be used by any user who can activate $s$. Note that in $H_{o}$, a user who can activate $s$ can also activate $r$ and hence acquire $P_{r}$. Hence, for this case, there is no appropriate transformation. Similarly, if $(s \geqslant r) \in H_{o}$, the only possible new relation is $\left(s \geqslant_{a} j\right)$. However, it is somewhat restrictive in the sense 


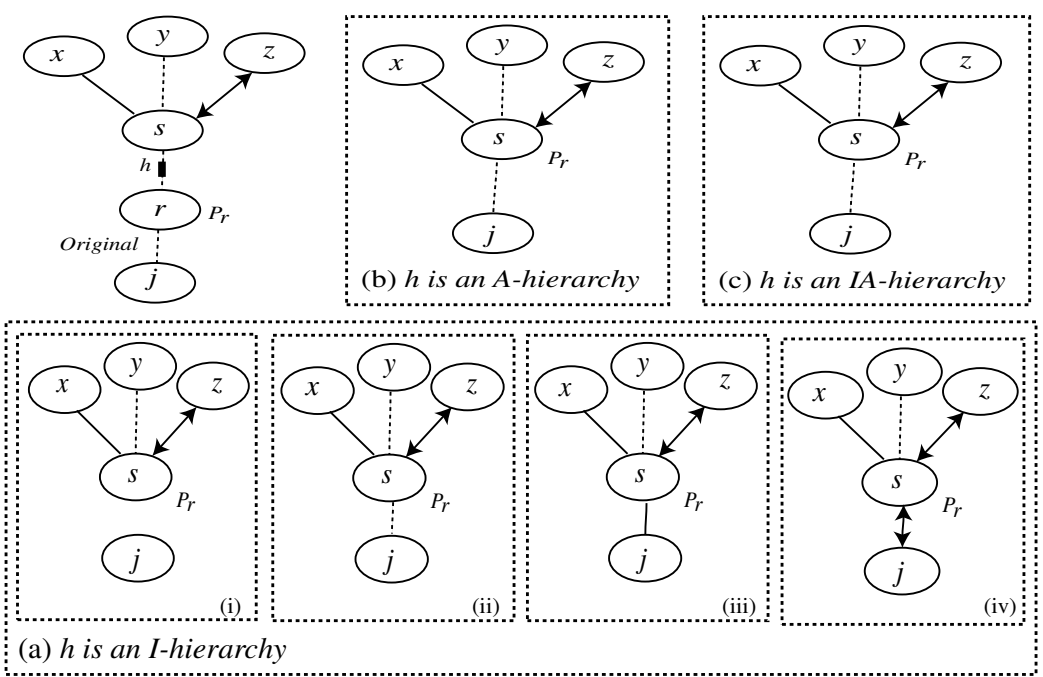

Fig. 10. Deletion of role $r$ when $(r \geqslant a j)$

that, in $H_{o}$, a user $u$ who can activate $s$ needs not explicitly activate $j$ to acquire its permissions, but in $H_{n}, u$ needs to activate $j$ to acquire its permissions.

\subsection{Partitioning of an Existing Role}

Sometimes, it is essential that an existing role be simply partitioned to change the semantics of the hierarchy. In particular, partitioning may indicate the requirement for separating the role's permissions into different subsets. We identify the following three ways to partition a role: (1) vertical partitioning: here a role is partitioned into a set of new roles that form a linear path with the permission set of the old role distributed among the new roles; (2) horizontal partitioning: here the role's permission set is partitioned into a number of disjoint sets, each of which is assigned to a new role; the new roles do not have any hierarchical relations between them; and (3) hybrid partitioning: here both vertical and horizontal partitioning are applied on the role, which result in an arbitrary hierarchy over the new roles. Fig. 11 illustrates these partitions.

In each case, the set of new roles replaces the partitioned role in the hierarchy. Once a role is partitioned, it is possible that an administrator completely redefines the hierarchical relationships in the part of the hierarchy above the partitioned role. Such a case requires offline redesign of the system. However, it may be necessary to retain the original hierarchical semantics as defined by criteria $\mathrm{C} 1$ and $\mathrm{C} 2$ (Table VII). Table X lays out various transformation characteristics of the three approaches with emphasis in retaining the original derived relation between $s$ and $j$. In particular, Table XI depicts cases where vertical partitioning creates a monotype linear path and hybrid partitioning creates multiple monotype linear paths. We discuss hybrid linear paths resulting from vertical and hybrid partitioning at the end of this section. Here, role $r$ of the original hierarchy $H_{o}$ is partitioned into a set of new roles $\mathrm{RP}=\left\{x_{1}, x_{2}, \ldots, x_{n}\right\}$. As usual, let $s$ and $j$ represent a senior 

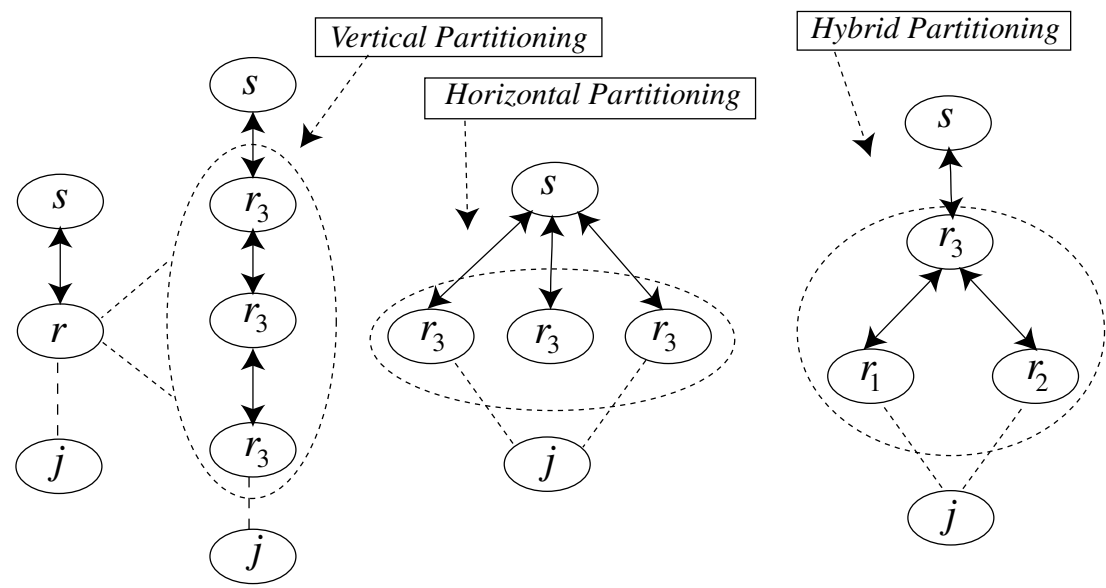

Fig. 11. Partitioning a role $r$ into three roles $r_{1}, r_{2}$ and $r_{3}$

and a junior of $r$.

Row 1 shows various hierarchy characteristics associated with the roles in RP. As already indicated above, in vertical partitioning, the new roles form a linear path. As shown in the table, if originally $\left(s \geqslant{ }_{i} r\right),(r\langle f\rangle j) \in H_{o}$, or $(s \geqslant r),\left(r \geqslant{ }_{i} j\right) \in H_{o}$, then in the new hierarchy $H_{n}$, the monotype hierarchy over the roles in RP should be of type $\geqslant_{i}$ or $\geqslant$. This is necessary to retain the original derived relation $\left(s \geqslant_{i} j\right)$ in the transformed hierarchy. If $(s \geqslant r),(r \geqslant j) \in H_{o}$ or $(s \geqslant r),(r \geqslant a j) \in H_{o}$, then the new linear path over the roles in RP should be of type $\geqslant$. Similarly, if $\left(s \geqslant{ }_{a} r\right)$, $(r\langle f\rangle j) \in H_{o}$, then the new linear path over the roles in RP should be of type $\geqslant_{a}$ or $\geqslant$.

The original semantics as defined by criteria $\mathrm{C} 1$ and $\mathrm{C} 2$ are ensured in the vertical partitioning by these transformations and by the new relations defined in rows 3 and 4 . For horizontal partitioning, the roles in RP are not hierarchically related. For hybrid partitioning, the roles in RP form multiple linear paths. The condition for the hybrid partitioning states that at least one linear path must allow inferring the derived relation $(s\langle f\rangle j)$ of $H_{n}$. For the linear path that maintains the original derived relation $(s\langle f\rangle j)$, we can use the transformations outlined for vertical partitioning in the if-then columns.

Entries in row 2 indicate the reassignments of the users in $U_{r}$ originally assigned to role $r$, to new role(s) in RP. The reassignments shown here are defined on the basis that the original access capabilities of the users are to be retained, although they may result in privilege escalation for some users. In practice, this may not be the actual case, and the relations among roles in the partition shown in row 1 may need to be accordingly adjusted. Rows 3 and 4 indicate how the roles $s$ and $j$ are related to the new roles in the partition. For a vertical partitioning approach, the original relation between $s$ and $j$ is used between $s$ and $x_{1}$, and $x_{n}$ and $j$, as 
Table XI. Transformation characteristics for different approaches to role partitioning

\begin{tabular}{|c|c|c|c|c|c|}
\hline \multicolumn{2}{|c|}{$\begin{array}{c}\text { Role } r \in \operatorname{Roles}\left(H_{o}\right) \\
\text { is partitioned into } \\
\mathrm{RP}=\left\{x_{1}, x_{2}, \ldots, x_{n}\right\} \\
\subset \operatorname{Roles}\left(H_{n}\right)\end{array}$} & \multicolumn{2}{|c|}{$\begin{array}{l}\text { Vertical Partitioning } \\
\text { (Monotype Linear Path) }\end{array}$} & $\begin{array}{l}\text { Horizontal } \\
\text { Partitioning }\end{array}$ & $\begin{array}{c}\text { Hybrid } \\
\text { Partitioning }\end{array}$ \\
\hline \multirow{6}{*}{1} & \multirow{6}{*}{$\begin{array}{l}\text { Hierarchy } \\
\text { characteristics }\end{array}$} & \multicolumn{2}{|c|}{$\begin{array}{c}L H=(\mathrm{RP},\langle f\rangle) \\
\text { (i.e. forms linear path) }\end{array}$} & \multirow{6}{*}{$\begin{array}{l}\text { No pair is } \\
\text { hierarchically } \\
\text { related }\end{array}$} & \multirow{6}{*}{$\begin{array}{c}H=(\mathrm{RP},[f])=\left\{L H_{1}, L H_{2}, \ldots,\right. \\
\left.L H_{n}\right\} \text { for } n>1(i . e . \text { a hierarchy } \\
\text { which is not a linear path }) \text { such } \\
\text { that Roles }\left(L H_{i}\right) \subset \mathrm{RP} \\
\text { Condition: } \\
\text { if }(s\langle f\rangle j) \text { is a derived relation in } \\
H_{o} \text { then at least one linear path } \\
L H_{i} \text { must allow deriving relation } \\
\quad(s\langle h\rangle j) \in H_{o} \text {. }\end{array}$} \\
\hline & & if & then & & \\
\hline & & $\begin{array}{c}\left(s \geqslant{ }_{i} r\right),(r\langle f\rangle j) \in H_{o} \text { or } \\
(s \geqslant r),\left(r \geqslant_{i} j\right) \in H_{o}\end{array}$ & $\langle f\rangle \in\left\{\geqslant_{i}, \geqslant\right\}$ & & \\
\hline & & $(s \geqslant r),(r \geqslant j) \in H_{o}$ or & $\langle f\rangle=\geqslant$ & & \\
\hline & & & & & \\
\hline & & $(s \geqslant a r),(r\langle f\rangle j) \in H_{o}$ & $\langle f\rangle \in\{\geqslant a, \geqslant\}$ & & \\
\hline 2 & $\begin{array}{l}\text { Reassignment } \\
\quad \text { of } U_{r}\end{array}$ & $\begin{array}{r}\text { For all } u \in \\
u \text { is assigned }\end{array}$ & & $\begin{array}{l}\text { For all } x \in \mathrm{RP}, \\
u \in U_{r}, u \text { is } \\
\text { assigned to } x\end{array}$ & $\begin{array}{c}\text { For all } x \in\left\{S_{L H_{1}}, S_{L H_{2}}, \ldots, S_{L H_{n}}\right\} \\
u \in U_{r}, \\
u \text { is assigned to } x\end{array}$ \\
\hline 3 & $\begin{array}{l}\text { Relation with } s \\
\text { where } \\
(s\langle f\rangle r) \in H_{O} \\
\end{array}$ & $\left(s\langle f\rangle x_{1}\right)$ & & $\begin{array}{l}\text { For all } x \in \mathrm{RP} \\
\qquad(s\langle f\rangle x)\end{array}$ & $\begin{array}{c}\text { For all } x \in\left\{S_{L H_{1}}, S_{L H_{2}}, \ldots, S_{L H_{n}}\right\} \\
(s\langle f\rangle x)\end{array}$ \\
\hline 4 & $\begin{array}{l}\text { Relation with } r \\
\text { where } \\
(r\langle f\rangle j) \in H_{o} \\
\end{array}$ & $\left(x_{n}\langle f\rangle j\right)$ & & $\begin{array}{c}\text { For all } x \in \mathrm{RP}, \\
\quad(x\langle f\rangle j)\end{array}$ & $\begin{array}{c}\text { For all } x \in\left\{J_{L H_{1}}, J_{L H_{2}}, \ldots, J_{L H_{n}}\right\} \\
(x\langle f\rangle j)\end{array}$ \\
\hline
\end{tabular}


indicated. Note that $x_{1}$ and $x_{n}$ are the senior-most and the junior-most roles of the new linear path created by the roles in RP. In case of horizontal partitioning, $s$ and $j$ are made senior and junior of each of the roles in RP. The case for hybrid partitioning is similar to that of the horizontal partitioning except that the role $s$ is made senior to the senior-most roles of each of the linear paths formed over the roles in RP, whereas $j$ is made the junior of each of junior-most roles of these linear paths.

So far, we have considered monotype linear components during the vertical and the hybrid partition. In general, the vertical partitioning and hybrid partitioning may result in hybrid linear path components. In such a case, the users originally assigned to the partitioned role need to be assigned to the role set from the partitioned set. First, consider the vertical partitioning. Here, the original users of the partitioned role is assigned to the maximal subset of the partition set, say MR, such that the roles in MR do not belong to any elements of the $\bigsqcup(H)$ of the senior-most role. In the case of the hybrid hierarchy, the original users are assigned to the set of roles that represents union of the MPs of individual elements of the complete LPDHH.

As indicated above, the need for such partitioning is primarily to restructure or redistribute permission sets in a hierarchy. Another reason for doing such partitioning may be because of the temporal properties. For example, a role may need to be vertically partitioned to arrange the temporal properties in such a way that the intervals associated with a senior role contain the intervals associated with the junior roles. Similarly, a horizontal partition may need to be done to create roles with distinct non-overlapping intervals. Furthermore, a hybrid partitioning may be needed to properly structure very complex temporal properties. Analysis of such partitioning based on temporal properties has been considered in detail in a slightly different context in [Joshi et al. 2005a], and also details the pros and cons of such partitioning and provides design guidelines.

\subsection{Edge Deletion and Insertion}

GTRBAC allows events of type (enable/disable $h$ ), which essentially adds or removes the hierarchical edge between a pre-specified pair of role. Using this event, periodicity and duration constraints on hierarchical relation can be expressed. Hence, edge deletion and insertion issues can be considered as related to the design of the time-based RBAC policies that includes hierarchical relations. In a generic non-temporal RBAC framework with hybrid hierarchy, edge deletion and insertion are important operations. However, both these operations can be viewed as operations on role addition and deletion discussed above. For instance, an edge deletion can be viewed as the deletion of the junior role of the edge and the addition of the same role with all the edges other than the deleted edge reinserted by considering the issues addressed earlier for role deletion. Similarly, an edge insertion can be viewed as a role addition operation(s), if either or both of the roles in the edge did not exist in the original hierarchy. Alternatively, if both the roles of the edges are present in the hierarchy, then edge addition can be viewed as removing the junior role of the edge and reinserting it with all its original hierarchical relations as well as the new relation. 


\section{RELATED WORK}

Several researchers have addressed issues related to inheritance semantics in RBAC [Giuri 1995], [Giuri 1996], [Moffett 1998], [Nyanchama and Osborn 1999], [Sandhu 1996], [Sandhu 1998]. Our earlier work has addressed issues concerning the inheritance relation when temporal properties are introduced [Joshi et al. 2002]. Furthermore, to the best of our knowledge, no work has been reported in the literature that thoroughly analyzes the coexistence of different types of hierarchical relations on a set of roles. In [Joshi et al. 2002], [Joshi et al. 2005b], we use the separate notion of hierarchy using permission-usage and role-activation semantics similar to the one proposed by Sandhu [Sandhu 1998] and strengthen Sandhu's argument that the distinction between the two semantics is very crucial. Sandhu's argument is based on the fact that the simple usage semantics is inadequate for expressing desired inheritance relation when certain dynamic SoD constraints are used between two roles that are hierarchically related, whereas, we emphasize the need for such distinction to capture the inheritance semantics in the presence of various temporal constraints. Sandhu's notion of activation hierarchy extending the inheritance hierarchy corresponds to the $I A$-hierarchy and our $A$-hierarchy corresponds to Sandhu's relation that relates two roles by activation hierarchy but not by inheritance semantics [Sandhu 1998]. In [Giuri 1995], [Giuri 1996], Giuri has proposed an activation hierarchy based on AND and OR roles. However, these AND-OR roles can be easily simulated within Sandhu's ER-RBAC96 model that uses usage and activation hierarchies, making Giuri's model a special case of ERRBAC96 [Sandhu 1998]. In order to address the needs of control principles in an organization, which include separation of duty, decentralization and supervision and review, Moffet et al. [Moffett 1998], [Moffett and Lupu 1999] have identified three types of hierarchies - is a hierarchy, activity hierarchy and supervision hierarchies. They show that for addressing more completely these control principles, we need a dynamic access control model and a hierarchy that allows restrictive inheritance as well as dynamic propagation of access rights [Moffett and Lupu 1999]. We believe that GTRBAC's temporal constraint framework with trigger and constraint enabling mechanisms, and temporal hierarchies can provide the modeling capabilities to address such dynamic issues. Nyanchama et. al. address the transformation of hierarchies in terms of addition, deletion and partitioning of roles in the context of access rights administration [Nyanchama and Osborn 1994]. However, the analysis is limited to monotype hierarchies and does not indicate how the transformations are affected by the presence of other constraints on hierarchical roles.

In [Sandhu et al. 1999], Sandhu et al. have presented ARBAC97 model for administrating RBAC policies using structural properties of RBAC96 hierarchy. Similarly, in [Crampton and Loizou 2003], Crampton et al. have proposed a Scoped Administration of RBAC (SARBAC) using the notion of an administration scope as a unit of administration to impose conditions on hierarchy operations. The aim of both the models has been to define administrative control by defining range or scope of control for the administration of roles and hierarchical relations. By using the flexible transformation primitives presented in Section 6, the development of a more complete RBAC administration model than the ARBAC97 and SARBAC models is possible and is left as a future work. Note that although both ARBAC97 and 
SARBAC emphasize hierarchy management, they do not consider the coexistence of SoD constraints and role hierarchies, and applies to monotype hierarchies only. Furthermore, role partitioning is a hierarchy transformation primitive that has not been addressed in the literature before.

\section{CONCLUSIONS AND FUTURE WORK}

In this paper, we have presented an extensive analysis of hybrid temporal role hierarchies for GTRBAC model. We have introduced the notion of uniquely activable set of a hierarchy that identifies access capabilities of a user assigned to a role in a hierarchy in a single session. The formal results we have presented allows determining uniquely activable sets for hybrid temporal role hierarchies and provide a basis for controlling privilege distribution to the users by restricting activable sets associated with the roles they are assigned to. The results related to the ACequivalence between different role hierarchies also show that, in cases where the principle of least privilege is not a concern, a monotype hierarchy may be used. Furthermore, as an A-hierarchy does not allow direct permission-inheritance, the results show that the $A$-hierarchy provides the most needed flexibility. In particular, an $A$-hierarchy allows DSoD constraints to be defined on hierarchically related roles. Furthermore, the inherit-all-permission semantics of $I$-hierarchy as well as $I A$-hierarchy has several pitfalls in terms of their ability to handle many organizational control principles [Moffett 1998]. We have also introduced a set of inference rules which can be employed to infer hierarchical relationships between pairs of roles that are not directly related. We have formally showed that the set of inference rules is sound and complete. In a complex hybrid hierarchy, these rules provide a formal basis for analyzing the permission acquisition and role activation semantics. We have also introduced the notion of conditioned derived relation that augments the three hierarchies ( $I, A$ and $I A$-hierarchies) and facilitates capturing much fine-grained derived permission acquisition and activation semantics within a hierarchy. We have also addressed the issue of hierarchy transformation with respect to role addition, deletion and partitioning. These transformations essentially form the basis for policy evolution in an organization. It is to be noted that transformations that retain original hierarchical semantics in a hybrid hierarchy need to be based on what type of additional role constraints exist or will be added in the hierarchy. The results presented in this paper provide a formal basis for developing administrative tools for the management of GTRBAC systems. Such security administrative functions are crucial for a well-planned, timely control of unauthorized accesses as well as for distributing least access capabilities to users in order to allow them to carry out their activities and at the same time minimize damage that may be caused by misuse of privileges. We plan to extend the present work in various directions. We also plan to develop an SQL-like language for specifying temporal properties for roles and to develop a prototype of such language on top of a relational DBMS. Using the results presented here, we plan to develop efficient security administration and management tools.

\section{ACKNOWLEDGMENTS}

acknowledgements 


\section{REFERENCES}

Barkley, J., Cincotta, A., Ferraiolo, D., Gavrila, S., and Kuhn, D. R. 1997. Role based access control for the world wide web. In Proceedings of 20th National Information System Security Conference. NIST/NSA.

Bertino, E., Bonatti, P. A., And Ferrari, E. 2001. Trbac: A temporal role-based access control model. ACM Transactions on Information and System Security 4, 3 (Aug.), 191-233.

Bertino, E. AND Ferrari, E. 1999. The specification and enforcement of authorization constraints in workflow management systems. ACM Transactions on Information and System Security 2, 1 (Feb.), 65-104.

Biskup, J., Flegel, U., And Karabulut, Y. 1998. Secure mediation: Requirements and design. In Proceedings of 12th Annual IFIP WG 11.3 Working Conference on Database Security. Chalkidiki, Greece.

Crampton, J. And Loizou, G. 2003. Administrative scope: A foundation for role-based administrative models. ACM Transsactions on Information System Security 6, 2, 201-231.

Ferraiolo, D. F., Gilbert, D. M., And Lynch, N. 1993. An examination of federal and commercial access control policy needs. In In Proceedings of NISTNCSC National Computer Security Conference. Baltimore, MD.

Gavrila, S. I. And Barkley, J. F. 1998. Formal specification for role based access control user/role and role/role relationship management. In Proceedings of the third ACM workshop on Role-based access control. Fairfax, Virginia.

Giuri, L. 1995. A new model for role-based access control. In Proceedings of 11th Annual Computer Security Application Conference. New Orleans, LA.

GiURI, L. 1996. Role-based access control: a natural approach. In RBAC '95: Proceedings of the first ACM Workshop on Role-based access control. ACM Press, New York, NY, USA, 13.

Jaeger, T. And Tidswell, J. E. 2001. Practical safety in flexible access control models. $A C M$ Transactions on Information and System Security 4, 2, 158-190.

Joshi, J. B. D., Aref, W., Ghafoor, A., And Spafford, E. H. 2001. Security models for web-based applications. Communications of the ACM 44, 2 (Feb.), 38-72.

Joshi, J. B. D., Bertino, E., And Ghafoor, A. 2002. Temporal hierarchies and inheritance semantics for gtrbac. In SACMAT '02: Proceedings of the seventh ACM symposium on Access control models and technologies. ACM Press, New York, NY, USA, 74-83.

Joshi, J. B. D., Bertino, E., Latif, U., And Ghafoor, A. 2005a. Analysis of expressiveness and design issues for a temporal role based access control model. IEEE Transactions on Dependable and Secure Computing. (accepted).

Joshi, J. B. D., Bertino, E., Latif, U., And Ghafoor, A. 2005b. Generalized temporal role based access control model. IEEE Transactions on Knowledge and Data Engineering 17, 1 (Jan.), $4-23$.

Joshi, J. B. D., Ghafoor, A., Aref, W., And Spafford, E. H. 2001. Digital government security infrastructure design challenges. IEEE Computer 34, 2 (Feb.), 66-72.

Koch, M., Mancini, L., And PArisi-Presicce, F. 2002. A graph-based formalism for rbac. $A C M$ Transactions on Information and System Security 5, 3, 332-365.

Moffett, J. D. 1998. Control principles and role hierarchies. In RBAC '98: Proceedings of the third ACM workshop on Role-based access control. ACM Press, New York, NY, USA, 63-69.

Moffett, J. D. And Lupu, E. C. 1999. The uses of role hierarchies in access control. In $R B A C$ '99: Proceedings of the fourth ACM workshop on Role-based access control. ACM Press, New York, NY, USA, 153-160.

Nyanchama, M. And Osborn, S. 1999. The role graph model and conflict of interest. ACM Transactions on Information and System Security 2, 1, 3-33.

Nyanchama, M. And Osborn, S. L. 1994. Access rights administration in role-based security systems. In Proceedings of the IFIP WG11.3 Working Conference on Database Security VII. North-Holland, 37-56. 
Osborn, S., Sandhu, R., And Munawer, Q. 2000. Configuring role-based access control to enforce mandatory and discretionary access control policies. ACM Transactions on Information and System Security 3, 2, 85-106.

Park, J. S., Sandhu, R., And Ahn, G. J. 2001. Role-based access control on the web. ACM Transactions on Information and System Security 4, 1 (Feb.), 37-71.

SAndHu, R. 1996. Role hierarchies and constraints for lattice-based access controls. Computer Security - Esorics'96, LNCS N. 1146, 65-79.

SAndhu, R. 1998. Role activation hierarchies. Proceedings of 2rd ACM Workshop on Role-based Access Control, 33-40.

Sandhu, R., Bhamidipani, V., And Munawer, Q. 1999. The arbac97 model for role-based administration of roles. ACM Transactions on Information and System Security 1, 2, 105-135.

Sandhu, R., Coyne, E. J., Feinstein, H. L., And Youman, C. E. 1996. Role-based access control models. IEEE Computer 29, 2, 38-47.

\section{Appendix A}

Proof of Lemma 4.1: Let $u$ be assigned to $S_{L h}$. By definition 4.4, $S_{L h}=$ $S_{L_{1}}, J_{L h}=J_{L_{2}}$ and $J_{L_{1}}=S_{L_{2}}$.

Case 1: First, consider $\left(\left\langle f_{1}\right\rangle,\left\langle f_{2}\right\rangle\right)=\left(\geqslant_{i},\left\langle f_{2}\right\rangle\right)$ s.t. $\left\langle f_{2}\right\rangle \in\left\{\geqslant, \geqslant_{a}\right\}$. As the first hierarchy is an $I$-hierarchy, $\bigsqcup\left(L_{1}\right)=S_{L_{1}}=x_{1}$ by theorem 4.1 and hence, none of the roles in $L_{2}$ can be activated by $u$. Therefore, we get $\bigsqcup(L h)=\bigsqcup\left(L_{1}\right)$. Similarly, let $\left(\left\langle f_{1}\right\rangle,\left\langle f_{2}\right\rangle\right)=\left(\left\langle f_{1}\right\rangle, \geqslant_{i}\right)$, where $\left\langle f_{1}\right\rangle \in\left\{\geqslant_{,} \geqslant_{a}\right\}$. As $L_{2}$ is an $I-$ hierarchy, $u$ cannot activate any junior roles, but $u$ acquires all the permissions of $L_{2}$ when he activates $S_{L_{2}}$. Because of $\left\langle f_{1}\right\rangle, u$ can activate $J_{L_{1}}\left(=S_{L_{2}}\right)$ and acquire all the permissions of $L_{2}$. Hence, $\bigsqcup(L h)=\bigsqcup\left(L_{1}\right)$. Therefore, if $\geqslant_{i} \in\left\{\geqslant, \geqslant_{a}\right\}$, then $\bigsqcup(L h)$ $=\bigsqcup\left(L_{1}\right)$.

Case 2: Consider $\left(\left\langle f_{1}\right\rangle,\left\langle f_{2}\right\rangle\right)=\left(\geqslant_{a}, \geqslant\right)$. Here, first we note that $\bigsqcup(L h)$ must contain the following:

1. all the activable elements of $L_{1}$, i.e. all elements of $\bigsqcup\left(L_{1}\right)$,

2. all the activable elements of $L_{2}$, i.e. all elements of $\bigsqcup\left(L_{2}\right)$, and

3. all the possible combinations of activable elements in $L_{1}$ and $L_{2}$.

From Theorem 4.1, $\bigsqcup\left(L_{1}\right)=2^{X_{1}} \backslash \phi$ and $\bigsqcup\left(L_{2}\right)=\left\{\left\{J_{L_{1}}\right\}, \ldots,\left\{x_{n}\right\}\right\}$. We need to show that $\bigsqcup(L h)=\bigsqcup\left(L_{1}^{U}\right) \cup \bigsqcup\left(L_{2}\right) \cup\left(\bigsqcup\left(L_{1}^{U}\right) \otimes \bigsqcup\left(L_{2}\right)\right)$ exactly contains the elements mentioned in 1-3. We see that $\bigsqcup\left(L_{1}\right)=\bigsqcup\left(L_{1}^{U}\right) \cup\left(\bigsqcup\left(L_{1}^{U}\right) \otimes J_{L_{1}}\right)$. But as $J_{L_{1}}=S_{L_{2}}, J_{L_{1}} \in \bigsqcup\left(L_{2}\right)$. Therefore, $\left(\bigsqcup\left(L_{1}^{U}\right) \otimes\left\{J_{L_{1}}\right\}\right) \subseteq\left(\bigsqcup\left(L_{1}^{U}\right) \otimes \bigsqcup\left(L_{2}\right)\right)$. Hence, elements of both $\bigsqcup\left(L_{1}\right)$ and $\bigsqcup\left(L_{2}\right)$ are in $\bigsqcup(L h)$. We further note that as there are no common roles in $L_{1}^{U}$ and $L_{2}$, hence, $\bigsqcup\left(L_{1}^{U}\right)$ and $\bigsqcup\left(L_{2}\right)$ are disjoint. It is easy to see that $\left(\sqcup\left(L_{1}^{U}\right) \otimes \sqcup\left(L_{2}\right)\right)$ consists of all the combinations of the activable sets of $\bigsqcup\left(L_{1}^{U}\right)$ and $\bigsqcup\left(L_{2}\right)$. $\left(\bigsqcup\left(L_{1}^{U}\right) \otimes \bigsqcup\left(L_{2}\right)\right)$ is disjoint from $\bigsqcup\left(L_{1}^{U}\right)$ and $\bigsqcup\left(L_{2}\right)$ as each of its role sets contains roles from the elements of both $\bigsqcup\left(L_{1}^{U}\right)$ and $\bigsqcup\left(L_{2}\right)$. (Hence, cardinality computation is simply $|\sqcup(L h)|=\left\|\bigsqcup\left(L_{1}^{U}\right)\left|\cup \|\left(L_{2}, t\right)\right| \cup\right.$ $\left|\left(\bigsqcup\left(L_{1}^{U}, t\right) \otimes \bigsqcup\left(L_{2}, t\right)\right)\right|$

Case 3:. Case for $\left(\left\langle f_{1}\right\rangle,\left\langle f_{2}\right\rangle\right)=\left(\geqslant, \geqslant_{a}\right)$ can be shown similarly.

Proof of Theorem 4.2: Let $u$ be assigned to $S_{L h}$. By definition 4.4, $S_{L h}=$ $S_{L_{1}}, J_{L h}=J_{L H_{2}}$ and $J_{L_{1}}=S_{L_{2}}$.

Case 1. $\left(\left\langle f_{1}\right\rangle=\geqslant_{i}\right)$ : As $L_{1}$ is an $I$-hierarchy, by reasons similar to that of the case for $\left(\left\langle f_{1}\right\rangle,\left\langle f_{2}\right\rangle\right)=\left(\geqslant_{i},\left\langle f_{x}\right\rangle\right)$ in the proof for Lemma 4.1, the result follows 
immediately.

Case 2. $\left(\left\langle f_{1}\right\rangle=\geqslant_{a}\right)$ : As $L_{1}$ is an $A$-hierarchy, if $\left\langle f_{x}\right\rangle=\geqslant_{i}$ then no roles below $S_{L x}$ can be in the set $\bigsqcup(L h)$; hence $\bigsqcup(L h)=\bigsqcup\left(L_{1}\right)$. If $\left\langle f_{x}\right\rangle=\geqslant$ then the case is similar to that of lemma 4.1.

Case 3:. As $L_{1}$ is an IA-hierarchy, if $\left\langle f_{x}\right\rangle=\geqslant_{i}$ then no roles below $S_{L 2}$ can be in the set $\bigsqcup(L h)$, hence $\bigsqcup(L h)=\bigsqcup\left(L_{1}\right)$. If $\left\langle f_{x}\right\rangle=\geqslant$ then the case is similar to that of Lemma 4.1 except for the fact that $L_{2}$ is not necessarily an $A$-hierarchy. Hence, by following similar reasoning, the result follows.

Proof of Theorem 4.3: Here, $\bigsqcup\left(H_{1}\right)$ has two parts, namely $S_{1}$ and $S_{2} . S_{1}$ constitutes the subset of $\bigsqcup(H)$ which is either from $L H_{1}$ or from $H_{1}$. Hence $S_{1}=$ $\left(\bigsqcup\left(L H_{1}\right) \cup \bigsqcup\left(H_{1}\right)\right)$. Similarly, $S_{2}$ constitutes the subset of $\bigsqcup(H)$ resulting from the combination of $\bigsqcup\left(L H_{1}\right)$ and $\bigsqcup\left(H_{1}\right)$. Here we note that there may be common elements. At the least, the senior-most role is common to both. Therefore, $S_{2}$ $=\left(\bigsqcup\left(L H_{1}\right) \backslash \mathrm{B} \otimes \bigsqcup\left(H_{1}\right) \backslash \mathrm{B}\right)$ contains all the combinations of the elements of the components $L H_{1}$ and $H_{1}$ (Here B represent the elements of $\bigsqcup\left(L H_{1}\right)$ and $\bigsqcup\left(H_{1}\right)$ that have common elements). However, $I=S_{1} \cup S_{2}$ may not still be the required activable set. This is because in both $L H_{1}$ and $H_{1}$, the same two roles may have a hierarchical relation (direct or derived) showing alternative relations between the roles. The result of the two alternative relations is that we may have a new derived relation (as discussed in Section 5). For example in $L H_{1}, x$ and $y$ may be related by an $A$-relation and hence appear together in an element of $\bigsqcup\left(L H_{1}\right)$, whereas in $H_{1}$, $x$ and $y$ may be related by an $I$-relation (or an $I A$-relation). As a result the derived relation between $x$ and $y$ becomes an $I A$-relation in $H$. Thus, $x$ and $y$ should not appear together in an element of $\sqcup(H)$. C determines exactly those elements in $\bigsqcup(H)$, that are $I A$-related (directly or derived), hence $\bigsqcup(\mathrm{H}, t)=\mathrm{I} \backslash \mathrm{C}$.

Proof of Theorem 4.4: We note that $H_{1}$ and $H_{2}$ have the same pair-wise related roles only differing in the hierarchical relation. Hence the hierarchy structure is the same and $H_{2}$ is a monotype whereas $H_{1}$ can be monotype or hybrid type. We prove this case wise. Let $X=\operatorname{Roles}\left(H_{2}\right)$.

Case 1. ( $H_{1}$ is monotype): Let us assume that $H_{2}$ is an $I$-hierarchy. Obviously, $P_{\max }\left(H_{2}\right)=P\left(S_{H_{2}}\right)=P(X)$. We have three cases for $H_{1}$. If $H_{1}$ is also $I$-hierarchy then by they are obviously same hierarchy. Let $H_{1}$ be an $A$-hierarchy. Then a user assigned to $S_{H_{1}}$ can activate all the roles at once in a session. Hence, $P_{\max }\left(H_{1}\right)$ $=P(X)=P_{\max }\left(H_{2}\right)$. Now, let $H_{1}$ be an $I A$-hierarchy. Thus, by activating role $S_{H_{2}}$, a user can acquire all the permissions of roles in $X$, i.e. $P_{\max }\left(H_{1}\right)=P\left(S_{H_{1}}\right)=$ $P(X)=P_{\max }\left(H_{2}\right)$. Thus, all monotype hierarchies are $A C$-equivalent.

Case 2. ( $H_{1}$ is hybrid type): Here it is possible that $H_{1}$ has a linear component $L H_{i}=\left(L H^{\prime}, L_{x}, L H_{\text {mid }}, L_{y}, L H^{\prime \prime}\right)$. In such a case, a user assigned to the senior-most role cannot acquire the permissions associated with roles in $\left(X_{y} \cup X\right.$ ") (considering $L_{y}=\left(X_{y},\left\langle f_{y}\right\rangle\right)$ and $\left.L^{\prime \prime}=\left(X ",\left\langle f^{\prime \prime}\right\rangle\right)\right)$ as $f_{x}$ is an $I$-relation. Thus, a user assigned to the senior-most role can acquire the permission set $P(X)$ in $H_{2}$, whereas he can acquire only the permission set $P\left(X \backslash\left(X_{y} \cup X\right.\right.$ ") $)$ in $H_{1}$. However, if such a component is not present then by Theorem 4.1 and 4.2 , the user acquires 


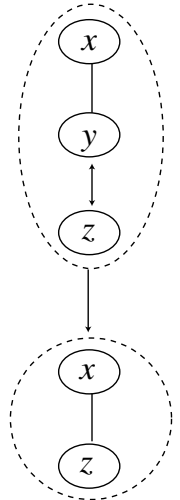

(a)

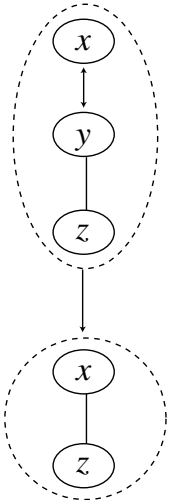

(b)

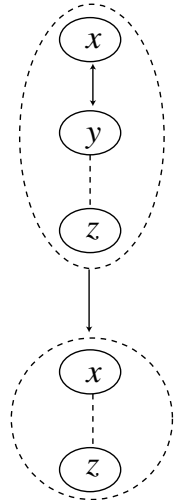

(c)

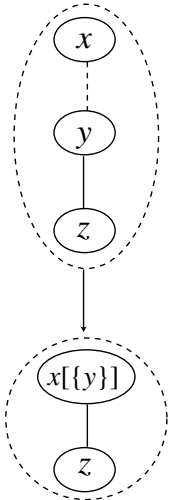

$(d)$

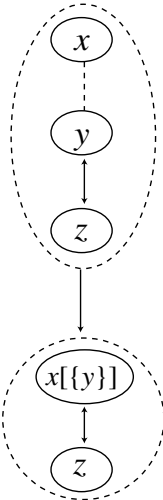

$(e)$

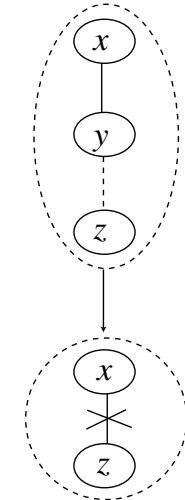

$(f)$

Fig. 12. Derived hierarchical relation for two consecutive types (rule R2)

the permission set $P(X)$ in both $H_{1}$ and $H_{2}$. Hence, we get the result.

\section{Appendix B}

Proof of Theorem 5.1 (Soundness of rules $R_{1} R_{4}$ ) : We prove this by taking all the possible cases of $h$ that can be derived from each rule. Let us assume that user $u$ is assigned only to the senior-most role $x$.

Case 1: $\boldsymbol{h}$ is derived from rule $R_{1}$ : Here $(x\langle f\rangle z)$ because $(x\langle f\rangle y)$ and $(y\langle f\rangle z)$. Let us consider $\langle f\rangle=\geqslant_{i}$. Assume that permission $p$ can be acquired through role $z$ at time $t$, i.e., can_be_acquired $(p, z, t)$ holds. As $\left(y \geqslant_{i} z\right), p$ can be acquired through role $y$ at time $t$ (by $c 1$ ). Again, as $\left(x \geqslant_{i} y\right), p$ can also be acquired through role $x$ at time $t$ (by $c 1$ ). Similarly, $\left(x \geqslant_{i} z\right)$ also indicates that $p$ can also be acquired through role $x$ at time $t$. Hence, the result follows. The cases for the $A$-hierarchy and $I A$-hierarchy can be shown similarly.

Case 2: $\boldsymbol{h}$ is derived from rule $R_{2}$ :. Fig. 12 depicts all the 6 possible combinations of $\left(x\left\langle f_{1}\right\rangle y\right)$ and $\left(x\left\langle f_{2}\right\rangle z\right)$. Fig. 12(a) - Fig. 12(e) correspond to the cases $R_{2.1(i)}, R_{2.1(i i}, R_{2.3}, R_{2.3(i)}$ and $R_{2.3(i i)}$ respectively. Rule $R_{2.1(i)}$ is straightforward. As both hierarchical relations allow permission inheritance, role $x$ can inherit all the permissions of role $z$. However, $u$ cannot activate role $y$ and hence he cannot activate role $z$. Thus, roles $x$ and $z$ are related by an $I$-relation only. The rules $R_{2.1(i i)}$ and $R_{2.2}$ can be shown in a similar way.

In rule $R_{2.3(i)}$ (refer to Fig. $12(\mathrm{~d})$ ), $x$ and $y$ are related by an $A$-relation, hence, $u$ can also activate role $y$. However, as $y$ and $z$ are related by an $I$-relation, $u$ cannot activate role $z$. $u$ can still acquire the permissions of role $z$ without activating it, but to do that he has to activate role y. Hence, we get a conditioned derived relation $\left(x[y]{ }_{i} z\right)$, as per definition 5.1. In rule $R_{2.3(i i)}$ (refer to Fig. 12(e)), $u$ can activate $z$. However, $u$ can also acquire $z$ 's permissions without activating it, but to do that he has to activate role $y$. Hence, we again get, as per definition 5.1, a conditioned derived relation $x[y](y) \geqslant z$. We note that the combination shown in Fig. 12(f) does not derive any relation between $x$ and $z$. Here, the $I$-relation 


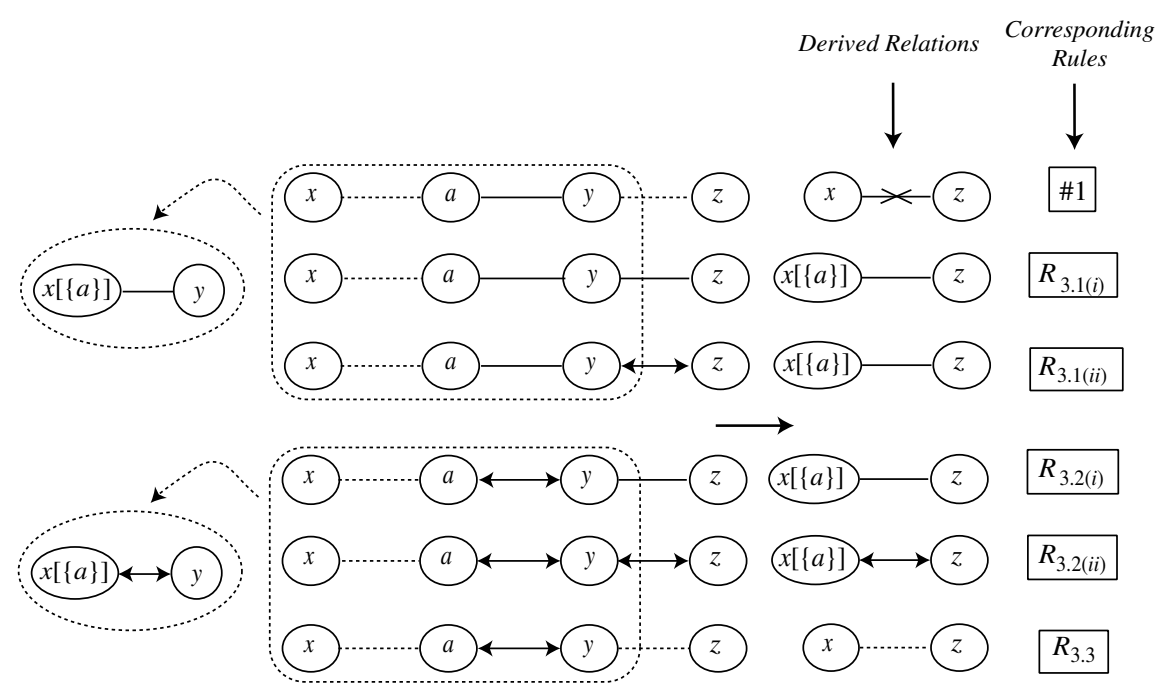

Fig. 13. Derived relations in a general linear using rules $R_{3}$ with set $\mathrm{B}$ is empty

between $x$ and $y$ prohibits activation of role $y$ and hence that of role $z$ by $u$. The $A$-relation between $y$ and $z$ prohibits inheritance of $z$ 's permissions through $y$ and hence $u$ cannot directly inherit $z$ 's permissions. Thus, $u$ can neither activate $z$ nor inherit its permissions. Hence, $x$ and $z$ are not hierarchically related. Thus, Fig. 12 shows all the possible cases in which a hierarchical relation (either direct or unconditioned derived) of one type follows another type (direct or derived). Thus, $R_{2}$ captures completely all such cases.

Case 3: $h$ is derived from rule $R_{3}: R_{3}$ deals with cases in which a conditioned derived relation is immediately followed by an unconditioned relation in a hierarchical path. Fig. 13 illustrates graphically all such possible combinations for $A=a$ and $B=\phi$. The combination labelled $\# 1$ that corresponds to $\left(x[A] \geqslant_{i} y\right) \wedge$ $\left(y \geqslant_{a} z\right)$ does not derive any new relation. This is because, $\left(a \geqslant_{i} y\right)$ is followed by $\left(y \geqslant{ }_{a} z\right)$ (see Fig. 12(f)). The remaining combinations correspond to the five cases of $R_{3}$ in Fig. 13.

In $R_{3.1(i)}$, corresponding to rule $R_{3.1 . a}$, the conditioned derived relation between $x$ and $y$ is an $I$-relation. As the relation between $y$ and $z$ is also an $I$-relation, $u$ can still acquire the permissions of $z$ through the activation of role $a$ as $z$ 's permissions can be acquired through $y$. Hence, the result is the conditioned derived relation $\left(x[\{a\}] \geqslant_{i} z\right)$. Similarly, in rule $R_{3.1(i i)}$, corresponding to rule $R_{3.1 . b}$, the presence of a more restrictive conditioned $I$-relation before the $I A$-hierarchy between $y$ and $z$ prohibits $u$ to activate $z$. But, because of the IA-hierarchy, $z$ 's permissions can be inherited through $y$ and hence through the activation of role $a$. Thus, the conditioned derived relation $\left(x[\{a\}] \geqslant_{i} z\right)$ holds. In rule $R_{3.2(i)}$, corresponding to rule $R_{3.2 . a}$, we see that a user assigned to $x$ can acquire $y$ 's permissions by activating role $a$. But the $I$-relation between $y$ and $z$ allows $z$ 's permissions to be inherited through $y$ and hence by the activation of role $a$. However, the same 


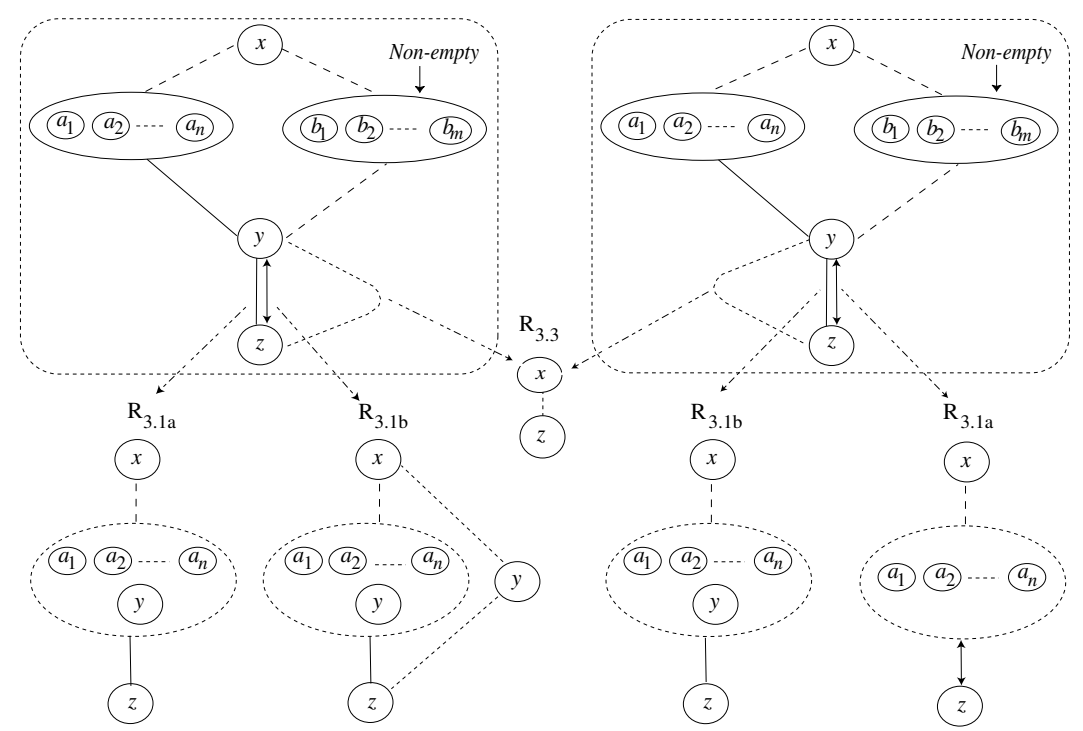

Fig. 14. Derived relations in a general linear hierarchy using rules $R_{3}$ with set $\mathrm{B}$ is non-empty

$I$-relation between $y$ and $z$ prohibits the user from activating $z$. Hence, the result is a conditioned $I$-relation $\left(x[\{a\}] \geqslant_{i} z\right)$. In rule $R_{3.2(i i)}$, corresponding to rule $R_{3.2 . b}$, because of $I A$-relation between $y$ and $z, u$ can activate $z$. At the same time, $u$ can also acquire through $y$ all of $z$ 's permissions by simply activating a role in A. Hence, the inferred rule is $(x[\{A\}] \geqslant z)$. In rule $R_{3.3}$, corresponding to rule $R_{3.3}, u$ can activate $y$ because of the $I A$-relation. The $A$-relation between $y$ and $z$ allows $u$ to activate $z$ also. However, because of the $A$-relation between $y$ and $z, u$ cannot acquire $z$ 's permissions without activating $z$. Hence the result is $\left(x \geqslant_{a} z\right)$. Note that it is not a conditioned derived relation. Again, we note that $R_{3}$ captures rules for deriving any new derived relations from all possible combinations of a conditioned derived relation followed by an unconditioned relation. Fig. 14 shows the same cases when set $B$ is non-empty. The proof is similar to that for the above case, except that whenever the new derived relation between $x$ and $z$ is an $A$ or $I A$-hierarchy, then the set is non-empty.

Case 4: $\boldsymbol{h}$ is derived from rule $R_{4}$ : The first rule $R_{4.1}$ is a trivial case and is straightforward. In rule $R_{4.2}$, we consider those alternate paths that have different unconditioned relations. When we have $\left(x \geqslant_{i} y\right)$ and $\left(x \geqslant_{a} y\right), u$ can directly inherit $y$ 's permissions through the first relation and at the same time $u$ can activate $y$ using the second relation, hence $(x \geqslant y)$. Similarly, when one of the two relations is $(x \geqslant y), u$ can inherit $y$ 's permissions directly as well as activate $y$. Thus, no matter what the other relation is we have the derived relation $(x \geqslant y)$. In rule $R_{4.3}$, we have various cases in which one relation is a conditioned derived relation and the other is an unconditioned relation, as depicted in Fig. 15. In $R_{4.3 a}$, both the hierarchical relations are of the same type. If the relation is $I$-hierarchy then the unconditioned relation allows direct inheritance of role $y$ 's permissions through role $x$; hence, 


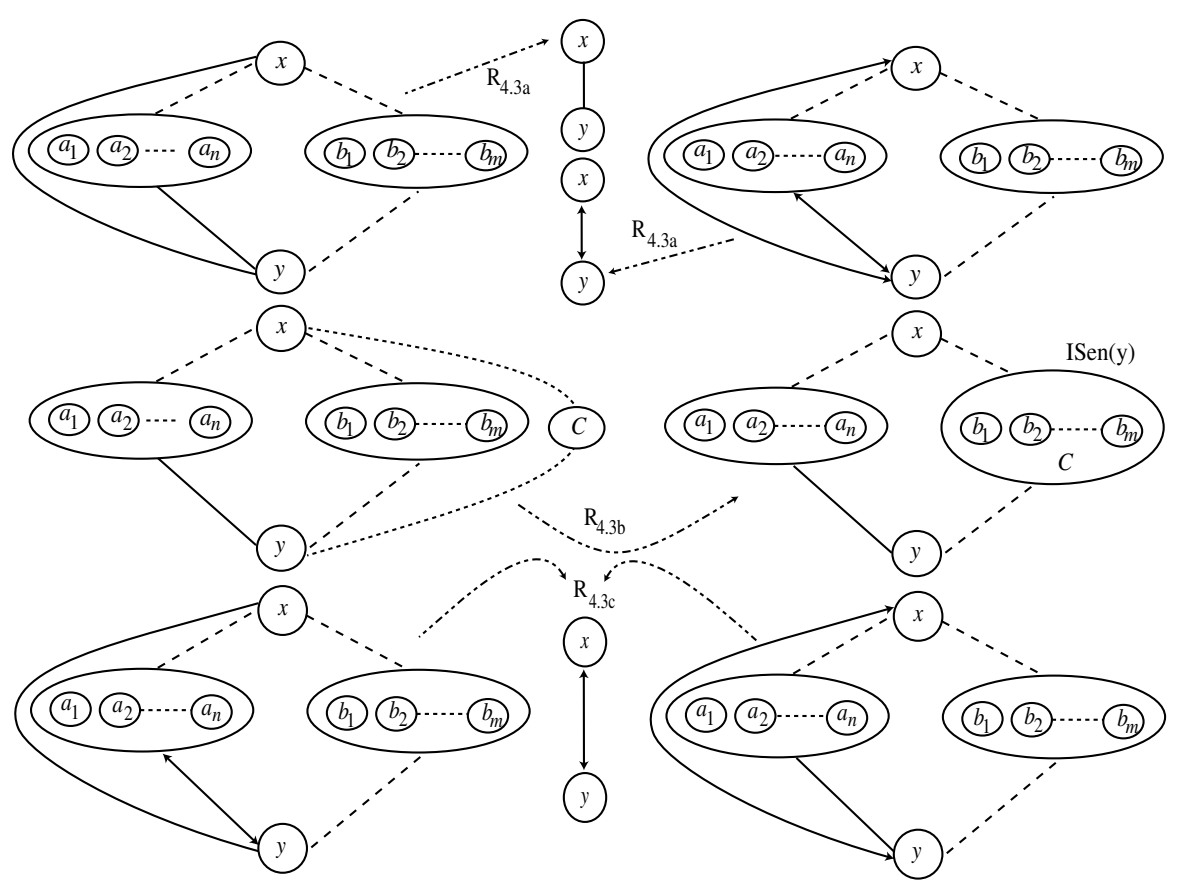

Fig. 15. Derived relations in a general linear hierarchy using rules $R_{3}$ with set $\mathrm{B}$ is non-empty

the resulting derived relation is an unconditioned $I$-relation. Similarly, when the relation is an $I A$-hierarchy, $y$ 's permissions are inherited by simply activating role $x$ using the unconditioned relation and hence the derived relation is not conditioned.

In rule $R_{4.3 b}$, the first relation is a conditioned one. When $\langle f\rangle$ is an $I$-relation, as in Fig. 15, then it means that through that hierarchical path, $u$ can acquire $y$ 's permission by activating a role in $A$. Furthermore, $x$ and $y$ also are related by $A$-hierarchy through sets $B$ and $C$. Note that $B$ and/or $C$ may contain role $x$ itself. The activation path is simply the union of the two activation paths of the two paths. The case where $\langle f\rangle$ is $I A$-relation, is similar. In rule $R_{4.3 c}$, we have $I$ and $I A$-relations, as shown in Fig. 15. In the first case, the first path allows $A$-relation between $x$ and $y$ through the conditioned relation, either through roles in $A$ or $B$, whereas the alternate path allows the $I$-relation. Hence, the derived relation is an $I A$-relation. In the second case, the $I A$-path allows both the semantics, hence the result is the $I A$-relation between $x$ and $y . R_{4.4}$ captures cases where both the alternate paths are conditioned (Fig. 16). Rule $R_{4.4 a}$ deals with the cases where both the alternate derived relations are same. In rule $R_{4.4 b}$, both the alternates have conditioned derived relations but are of different types: $I$ and $I A$-hierarchy. In the first case, there is a conditioned $I$-hierarchy portion on the first hierarchical path through $A_{1}$ and a conditioned $I A$-hierarchy portion on the second hierarchical path through $A_{2}$. As the path through $A_{2}$ provides $I A$-relation, this means $A_{2}$ provides both $I$ and $A$-relations. Now, by taking union of the $I$ and $A$-paths separately, we get the result. The second case is the similar to the first one. Thus, the inference 


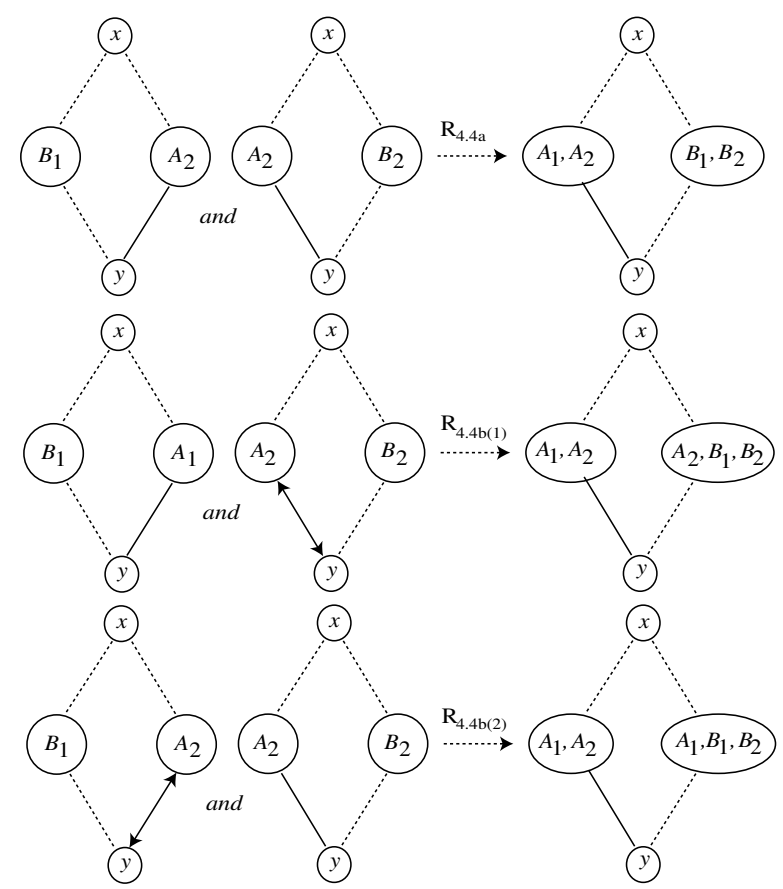

Fig. 16. Derived relations for rules $\mathrm{R}_{4.4}$

rules covers all possible combinations. Hence, the result follows.

To prove Theorem 5.2, we first prove the following lemmas:

Lemma 5.1 (Completeness of rules $R_{1}$ in monotype linear hierarchy): Given a monotype linear hierarchy $L$, rule $R_{1}$ is complete with respect to $L$; That is, if for any pair of roles $x, z \in \operatorname{Roles}(L), \neg L\left[R_{1}\right] \vDash h_{x, z}$, then $L \nsim L \cup\left\{h_{x, z}\right\}$, i.e., the hierarchies $L$ and $L^{\prime}=L \cup\left\{h_{x, z}\right\}$ are not authorization consistent.

Lemma 5.2 (Completeness of rules $R_{1}-R_{3}$ in hybrid linear hierarchy): Given a hybrid linear hierarchy $L h$, rules $R_{1}-R_{3}$ are complete with respect to $L h$; That is, if for any pair of roles $x, z \in \operatorname{Roles}(L h), \neg L h\left[R_{1}-R_{3}\right] \vDash h_{x, z}$, then $L h$ $\nsim L h \cup h_{x, z}$, i.e., the hierarchies $L h$ and $L h^{\prime}=L h \cup h_{x, z}$ are not authorization consistent.

Proof of Lemma 5.1: . Assume otherwise, i.e., there exists a relation $h_{x, z}=$ $(x\langle f\rangle z)$, such that $\neg L\left[R_{1}\right] \vDash h_{x, z}$, but $L \approx L '$. As $\neg L\left[R_{1}\right] \vDash h_{x, z}$, it implies that there exists no $y$ such that $(x\langle f\rangle y) \wedge(y\langle f\rangle z)$ holds under $L$. It is easy to see that $(x\langle f\rangle y)$ $\wedge(y\langle f\rangle z)$ cannot hold iff the following hold under $L$ :

1. for all $y$ such that $(x\langle f\rangle y)$, we have $\neg(y\langle f\rangle z)$, or

2. for all $y^{\prime}$ such that $\left(y^{\prime}\langle f\rangle z\right)$, we have $\neg\left(x\langle f\rangle y^{\prime}\right)$,

Let us first consider $\langle f\rangle \in \geqslant_{i}$, i.e. $h_{x, z}=\left(x \geqslant_{i} z\right)$. Note that there is no direct hierarchical relation between $x$ and $z$ as $h_{x, z}$ is not in $L$. The first condition above 
indicates that $x$ can inherit permissions from $y$ because of the relation $\left(x \geqslant_{i} y\right)$ but because $\neg(y\langle f\rangle z)$ also holds for all such $y, x$ cannot inherit from $z$. Similarly, the second condition indicates that none of the roles that are senior to $z$ is a junior of $x$. Therefore, $x$ cannot inherit $z$ 's permissions. Hence, it follows that $u$ cannot acquire permissions from $z$ under hierarchy $L$. But as $h_{x, z}$ is in $L$ ', $u$ can acquire $z$ 's permissions under $L^{\prime}$. Hence, it follows that $L \nsim L$ ', contradicting our assumption. Thus, if $\neg L\left[R_{1}\right] \vDash h_{x, z}$, then $L$ and $L$ ' are not authorization consistent.

Proof of Lemma 5.2:. As Lh is a hybrid linear hierarchy, by definition 4.2 we can write $L h=\left(L_{1}, L_{2}, \ldots, L_{n}\right)$, where each $L_{i}$ is a monotype linear hierarchy. Let $L_{i}$ be $\left(x_{i(1)}\left\langle f_{i}\right\rangle x_{i(2)}\left\langle f_{i}\right\rangle \ldots\left\langle f_{i}\right\rangle x_{i(|L i|)}\right)$. Then by Lemma 5.1, we get the derived relations $\left(x_{i\left(\pi_{i}\right)}\left\langle f_{i}\right\rangle x_{i\left(\eta_{i}\right)}\right)$ for $1 \leq \pi_{i} \leq\left(\left|L_{i}\right|-2\right)$ and $3 \leq \eta_{i} \leq\left|L_{i}\right|$. For each linear component, the derived set is complete as per Lemma 5.1. But, we know that for $\mathrm{i}=2$ to $n, S_{L_{i}}=J_{L_{(i-1)}}$ by definition 4.2 . To show completeness of $R_{1}-R_{3}$ with respect to $L h$, we show that any derived relation between a role in $L_{1}$ with that of a role in $L_{j}$ for $2 \leq j \leq n$ can be inferred from $R_{1}-R_{3}$. To do that, we use induction on the following hierarchical chain from an arbitrary role $x_{1\left(\pi_{1}\right)}$ in $L_{1}$ to an arbitrary role $x_{n\left(\eta_{n}\right)}$ in $L_{n}$ :

$$
L h_{c}=x_{1(\pi 1)}\left\langle f_{1}\right\rangle x_{1\left(\left|L_{1}\right|\right)}\left\langle f_{2}\right\rangle x_{2\left(\left|L_{2}\right|\right)} \ldots x_{(n-1)\left(\left|L_{(n-1)}\right|\right)}\left\langle f_{n}\right\rangle x_{n\left(\eta_{i}\right)}
$$

Note that each hierarchical relation in this chain is a relation derived in each component using $R_{1}$.

Basis: Consider $n=2$, i.e., $L h_{c}=x_{1\left(\pi_{1}\right)}\left\langle f_{1}\right\rangle x_{1\left(\left|L_{1}\right|\right)}\left\langle f_{2}\right\rangle x_{2\left(\eta_{2}\right)}$. In the proof for soundness of $R_{2}$ (Fig. 12), we showed that $R_{2}$ captures all possible combinations of $\left\langle f_{1}\right\rangle$ and $\left\langle f_{2}\right\rangle$. Hence, as $R_{2}$ is sound, by employing an argument similar to the proof of Lemma 5.1, it follows that $R_{2}$ is complete with respect to $L h_{c}$. Note that as $x_{1\left(\left|L_{1}\right|\right)}\left\langle f_{2}\right\rangle x_{2\left(\left|L_{2}\right|\right)}$ by $R_{1}$, rules $R_{1}-R_{3}$ are complete for $L h_{c}=x_{1\left(\pi_{1}\right)}\left\langle f_{1}\right\rangle x_{1\left(\left|L_{1}\right|\right)}\left\langle f_{2}\right\rangle x_{2\left(\left|L_{2}\right|\right)}$

Induction Hypothesis: Assume that rules $R_{1}-R_{3}$ are complete for $L h_{c}=x_{1\left(\pi_{1}\right)}$ $\left\langle f_{1}\right\rangle x_{1\left(\left|L_{1}\right|\right)}\left\langle f_{2}\right\rangle x_{2\left(\left|L_{2}\right|\right)} \ldots x_{(n-1)\left(\left|L_{(n-1)}\right|\right)}$. Now we need to show that they are complete for $L h c=x_{1}(\pi 1)\left\langle f_{1}\right\rangle x_{1\left(\left|L_{1}\right|\right)}\left\langle f_{2}\right\rangle x_{2\left(\left|L_{2}\right|\right)} \ldots x_{(n-1)\left(\left|L_{(n-1)}\right|\right)}\left\langle f_{n}\right\rangle x_{n(\eta n)}$. As $R_{1}-R_{3}$ are complete $L h_{c}=x_{1\left(\pi_{1}\right)}\left\langle f_{1}\right\rangle x_{1\left(\left|L_{1}\right|\right)}\left\langle f_{2}\right\rangle x_{2\left(\left|L_{2}\right|\right)} \ldots x_{(n-1)\left(\left|L_{(n-1)}\right|\right)}$, we can deduce a derived relation between $x_{1\left(\pi_{1}\right)}$ and $x_{(n-1)\left(\left|L_{(n-1)}\right|\right)}$, which is either

1. $\left(x_{1\left(\pi_{1}\right)}\langle f\rangle x_{(n-1)\left(\left|L_{(n-1)}\right|\right)}\right)$ where $\langle f\rangle \in\left\{\geqslant_{i}, \geqslant, \geqslant a\right\}$, or

2. $\left(x_{1\left(\pi_{1}\right)}[A](B)\langle f\rangle x_{(n-1)\left(\left|L_{(n-1)}\right|\right)}\right)$, where $\langle f\rangle \in\left\{\geqslant_{i}, \geqslant\right\}$ and

$$
A, B \subseteq\left\{x_{1\left|L_{1}\right|}, x_{2\left|L_{2}\right|}, \ldots, x_{(n-1)\left|L_{(n-1)}\right|}\right\} .
$$

Assume, $\left(x_{1\left(\pi_{1}\right)}\langle f\rangle x_{(n-1)\left(\left|L_{(n-1)}\right|\right)}\right)$ i.e. the derived relation is unconditioned. But as pointed out in the proof for soundness of $R_{1}-R_{4}$, rule $R_{2}$ can be employed over $\left(x_{1(1)}\langle f\rangle x_{\left.(n-1) \mid L_{(n-1)}\right)}\right)$ and $\left(x_{(n-1)\left|L_{(n-1)}\right|}\left\langle f_{n}\right\rangle x_{n\left|L_{n}\right|}\right)$ to derive any relation between $x_{1\left(\pi_{1}\right)}$ and $x_{n\left|L_{n}\right|}$ (i.e., as indicated earlier, all combinations of relations $\langle f\rangle$ and $\left\langle f_{n}\right\rangle$ are captured by $R_{2}$ ). As already argued in the induction basis, $R_{2}$ is complete for such cases.

Now suppose the derived relation is $\left(x_{1\left(\pi_{1}\right)}[A](B)\langle f\rangle x_{(n-1)\left|L_{(n-1)}\right|}\right)$. We know that $\langle f\rangle$ can only be $\geqslant_{i}$ or $\geqslant$. But as mentioned in the proof of the soundness theorem, $R_{3}$ completely captures all the combinations where an unconditioned relation 
follows a conditioned derived relation. This argument can be easily extended to any arbitrary pair of roles. Hence, using argument similar to that in the proof of Lemma 5.1, it follows that $R_{1}-R_{3}$ is complete with respect to a (derived) hybrid linear hierarchy $L h_{c}$. Using the same technique we can easily prove that a relation between any role of $L_{i}$ with that of $L_{j}, 1 \leq i<j$ is completely determined by rules $R_{1}-R_{3}$. Hence, $R_{1}-R_{3}$ is complete with respect to the hybrid linear hierarchy $L h$.

Theorem 5.2 (Completeness of rules $R_{1}-R_{4}$ ): . We prove this by considering various cases of $H$.

Case 1: H is a linear hierarchy:. If it is a monotype linear hierarchy, we can see that only $R_{1}$ applies as all other rules involve more than one hierarchy types or more than one relations between the same pair of roles. Hence from Lemma 4.1, it follows that $R_{1}-R_{4}$ is complete with respect to $H$. If $H$ is a hybrid linear hierarchy then that means only one relation can exit between a pair of roles, i.e. there are no alternative hierarchical paths between the roles. Thus, only rules $R_{1}$ through $R_{3}$ apply. Hence, from Lemma 5.2, it follows that $R_{1}-R_{4}$ is complete with respect to $H$.

Case 2: H is a not a linear hierarchy:. By definition 4.3, we can write $H=$ $\left(L H_{1}, L H_{2}, \ldots, L H_{m}\right)$, where each component $L H_{i}$ is a linear hierarchy (hybrid or monotype). From Lemma 5.1 and Lemma 5.2, we can see that for each $L H_{i}$, rules $R_{1}-R_{3}$ are complete. The only remaining case is the case where two or more hierarchical paths can exist between a pair of roles. Such a case can occur only in a hierarchy that is not a linear hierarchy. Now, we need to show that when multiple hierarchical paths exist between a pair of roles, rule $R_{4}$ provides a basis for inferring all derivable relations. First, let us consider the following $n$ hierarchical relations between roles $x$ and $y: h_{x y\left(\pi_{1}\right)}, h_{x y\left(\pi_{2}\right)}, \ldots, h_{x y\left(\pi_{n}\right)}$, which corresponds to the linear components $L H_{\pi_{1}}, L H_{\pi_{2}}, \ldots, L H_{\pi_{n}}$ for $n \leq m$ and $\left\{\pi_{1}, \pi_{2}, \ldots, \pi_{n}\right\} \subseteq\{1,2, \ldots, m\}$. It is easy to see that each of these relations between $x$ and $y$ can be completely derived using rules $R_{1}$ through $R_{3}$ as each is derived within a linear component. Now, we show that $R_{4}$ completely covers all the possible derived rules that can be inferred by using these relations between $x$ and $y$. Again, we use induction.

Basis: Let $n=2$. Then, we have only two relations between $x$ and $y$ deduced in components $L H_{\pi_{1}}, L H_{\pi_{2}}$, which are $h_{x y\left(\pi_{1}\right)}, h_{x y\left(\pi_{2}\right)}$. We note that $h_{x y\left(\pi_{1}\right)}, h_{x y\left(\pi_{2}\right)}$ can be any of the unconditioned relation or the conditioned derived relation. As noted in the proof of the Soundness Theorem, all possible combination is captured by the rules in $R_{4}$. Hence, applying argument similar to that used in the proof for Lemma 5.1, it follows that $R_{4}$ is complete with respect to the two relations $h_{x y\left(\pi_{1}\right)}$, $h_{x y\left(\pi_{2}\right)}$ between the same pair.

Induction hypothesis: Assume that $R_{4}$ is complete w.r.t. the $(n-1)$ relations between the same pair of roles $x$ and $y$.

Induction: Let $h_{x y(\pi)}$ be the derived relation between $x$ an $y$ from $n$ - 1 different relations between them using rule $R_{4}$. Now we have two relations between $x$ and $\mathrm{y}$ : $h_{x y(\pi)}$ and $h_{x y(\pi n)}$. It is easy to see that $h_{x y(\pi)}$ is one of the unconditioned relation because of the application of rules $R_{4.1}, R_{4.2}, R_{4.3 a}$ and $R_{4.3 c}$, or the conditioned derived relation with possibly bigger set on which the relation is conditioned on as is possible because of $R_{4.3 b}$ and $R_{4.4}$. But the same rules also apply to $h_{x y(\pi)}$ 
and $h_{x y(\pi n)}$. This is because, the rules in $R_{4}$ covers all possible combinations of conditioned and/or unconditioned alternate relations. Thus, by applying an argument similar to that used to prove Lemma 5.1, it follows that $R_{4}$ is complete for all $n$ different relations. Hence, it follows that rules $R_{1}-R_{4}$ is complete with respect to a hierarchy.

\section{Appendix C}

Here, we present an alternate way to compute the $\bigsqcup(H)$ of a hierarchy. The key issue is that a hierarchy relation generates a partial order of role sets [posets]. That is, for a role set $R$ and $\langle f\rangle \in\left\{\geqslant_{\mathrm{i}}, \geqslant_{\mathrm{a}}, \geqslant\right\}$, a hierarchy $H=(R,\langle f\rangle)$ denotes a poset of roles, which means for $x, y, z \in R$

1. $(x\langle f\rangle x)$ (We assume that a role is senior to itself)

2. $(x\langle f\rangle y)$ and $(y\langle f\rangle x)$ implies $x=y$;

3. $(x\langle f\rangle y)$ and $(y\langle f\rangle z)$ implies $x\langle f\rangle z$;

[reflexivity property] [anti-symmetry property] [transitivity property]

Note that cases (1) and (2) are actually not allowed in the RBAC models; however, for mathematically viewing a role hierarchy as a poset, it does not introduce any problem. Case (3) is implied from the definition of each hierarchy type. Based on this, a hybrid hierarchy can be considered as a combination of the three posets of a role set related to the three types of hierarchies. Furthermore, a set $X$ is called a chain or total order if, for all $x, y \in X$, either $(x\langle f\rangle y)$ or $(y\langle f\rangle x)$; and $X$ is called an antichain if, for all $x, y \in X,(x\langle f\rangle y)$ only if $(x=y)$. Let antichain_set $(H)$ be function that computes the set of all antichains of $H$. Furthermore, let can_activate_set(ASet) be the set of roles that a user assigned to the senior-most role in $H$ can activate according to the $A$-hierarchy relation defined in $A S e t$, which is a subset of $H$. Let $H_{s}$ denote the senior-most role in $H$. Now, the computation of $\bigsqcup(H)$ is given by the following theorem:

THEOREM 8.1. $(\bigsqcup(H)$ using anti_chain $)$ : Given a hybrid hierarchy $H$ over roles R. $\bigsqcup(H)=$ antichain_set $($ ISet $) \bigcap 2^{\text {can_activate_set }(A S e t)}$

where ASet $=\left(R, \geqslant_{a}\right)$ and ISet $=\left(R, \geqslant_{i}\right)$ are posets defined on $R$ that satisfy the following criteria:

a. $\forall(x\langle f\rangle y) \in H,\left(\langle f\rangle \in\left\{\geqslant_{a}, \geqslant\right\}\right) \rightarrow\left(\left(x \geqslant{ }_{a} y\right) \in A S e t\right)$

b. $\forall(x\langle f\rangle y) \in H,\left(\langle f\rangle \in\left\{\geqslant_{i}, \geqslant\right\}\right) \rightarrow((x \geqslant i y) \in I$ Set $)$

Proof: The proof follows as $\bigsqcup(H)$ contains only those incomparable sets of roles (antichains), indicated by antichain_set (ISet), that can be activated together, as indicated by the chains in the ASet. For instance, when there are no I-hierarchy, the antichain_set (ISet) is a set of all the combinations of the elements of $R$. Based on this theorem, steps to complete $\bigsqcup(H)$ will be as follows:

1. Given original hierarchy, create two hierarchies

a. An A-hierarchy (ASet) containing edges for each $A$ and $I A$ relation in $H$

b. An $I$-hierarchy (ISet) containing edges for each $I$ and $I A$ relation in $H$

2. Compute the antichain_set for the ISet

3. Remove from ISet any role set that cannot be activated by the user assigned 
to $H_{s}$ as per the ASet.

This approach characterizes the Uniquely Activable Set in a much simpler way. However, to compute the $\bigsqcup(H)$, it requires computing the antichain_set and the set of roles that the user assigned to $H_{S}$ can activate. The difference between this approach and the one presented in section 4 is that the former computes $\bigsqcup(H)$ of the entire hierarchy incrementally from that of the sub-hierarchies, while the approach described above operates on the entire hierarchy. The approach described in section 4 hence facilitates the maintenance of the $\bigsqcup(H)$ of the hierarchy incrementally. That is, a tool can be easily designed to compute and maintain the $\bigsqcup(H)$ information for any sub-hierarchy and use that to reconstruct the $\bigsqcup(H)$ of the entire hierarchy. The $\bigsqcup(H)$ information for each role in the hierarchy is needed in order to support the authorization decision process. This would not be easy using the approach described here. Furthermore, the computation of the antichain-set for a general $I$-hierarchy itself is not a straightforward task. The approach in section 4 essentially provides one incremental way to compute the antichains that form the $\bigsqcup(H)$ of the given hierarchy. 